\title{
Search for new physics in final states with an energetic jet or a hadronically decaying $W$ or $Z$ boson and transverse momentum imbalance at $\sqrt{s}=13 \mathrm{TeV}$
}

\author{
A. M. Sirunyan et al. ${ }^{*}$ \\ (CMS Collaboration)
}

(Received 6 December 2017; published 21 May 2018)

\begin{abstract}
A search for new physics using events containing an imbalance in transverse momentum and one or more energetic jets arising from initial-state radiation or the hadronic decay of $W$ or $Z$ bosons is presented. A data sample of proton-proton collisions at $\sqrt{s}=13 \mathrm{TeV}$, collected with the CMS detector at the LHC and corresponding to an integrated luminosity of $35.9 \mathrm{fb}^{-1}$, is used. The observed data are found to be in agreement with the expectation from standard model processes. The results are interpreted as limits on the dark matter production cross section in simplified models with vector, axial-vector, scalar, and pseudoscalar mediators. Interpretations in the context of fermion portal and nonthermal dark matter models are also provided. In addition, the results are interpreted in terms of invisible decays of the Higgs boson and set stringent limits on the fundamental Planck scale in the Arkani-Hamed, Dimopoulos, and Dvali model with large extra spatial dimensions.
\end{abstract}

DOI: 10.1103/PhysRevD.97.092005

\section{INTRODUCTION}

Several astrophysical observations [1-3] provide compelling evidence for the existence of dark matter (DM), a type of matter not accounted for in the standard model (SM). To date, only gravitational interactions of DM have been observed, and it remains unknown if DM has a particle origin and could interact with ordinary matter via SM processes. However, many theoretical models have been proposed in which DM and SM particles interact with sufficient strength that DM may be directly produced with observable rates in high energy collisions at the CERN LHC. While the DM particles would remain undetected, they may recoil with large transverse momentum $\left(p_{\mathrm{T}}\right)$ against other detectable particles, resulting in an overall visible $p_{\mathrm{T}}$ imbalance in a collision event. This type of event topology is rarely produced in SM processes and therefore enables a highly sensitive search for DM. Similar event topologies are predicted by other extensions of the SM, such as the Arkani-Hamed, Dimopoulos, and Dvali (ADD) model [4-8] of large extra spatial dimensions (EDs).

This paper describes a search for new physics resulting in final states with one or more energetic jets and an imbalance in $p_{\mathrm{T}}$ due to undetected particles. The jets are the

*Full author list given at the end of the article.

Published by the American Physical Society under the terms of the Creative Commons Attribution 4.0 International license. Further distribution of this work must maintain attribution to the author(s) and the published article's title, journal citation, and DOI. Funded by SCOAP. result of the fragmentation and hadronization of quarks or gluons, which may be produced directly in the hard scattering process as initial-state radiation or as the decay products of a vector boson $V$ ( $W$ or $Z)$. These final states are commonly referred to as "monojet" and "mono- $V$." Several searches have been performed at the LHC using the monojet and mono- $V$ channels [9-15]. This analysis makes use of a data sample of proton-proton (pp) collisions at $\sqrt{s}=13 \mathrm{TeV}$ collected with the CMS detector at the LHC, corresponding to an integrated luminosity of $35.9 \mathrm{fb}^{-1}$. This sample is approximately three times larger than the one used in Ref. [14]. The analysis strategy is similar to that of previous CMS searches and simultaneously employs event categories to target both the monojet and mono- $V$ final states. In an improvement compared to previous searches, in this paper, revised theoretical predictions and uncertainties for $\gamma+$ jets, $Z+$ jets, and $W+$ jets processes based on recommendations of Ref. [16] are used. In addition to interpretations in the context of simplified DM models [17-19], in this paper, the results are further studied in the context of the fermion portal (FP) dark matter model [20], the light nonthermal DM model [21,22], and the ADD model.

In many simplified DM models, DM particles are assumed to be Dirac fermions that interact with SM particles through a spin- 1 or spin-0 mediator [18,20,23-38]. These interactions are classified into four different types, depending on whether the mediator is a vector, axial-vector, scalar, or pseudoscalar particle. The spin- 0 mediators are assumed to couple to the SM particles via Yukawa couplings. The SM Higgs boson is a specific example of a scalar mediator that 

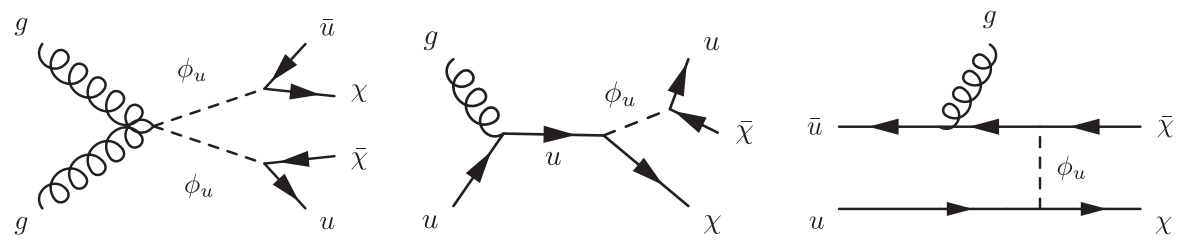

FIG. 1. Examples of Feynman diagrams of the main production mechanisms at the LHC of DM particles in association with a quark or gluon in the fermion portal model providing multijet (left) and monojet (middle, right) signatures.

may couple to the DM particles. Combined results of the direct searches for invisible Higgs bosons have been presented by both the ATLAS and CMS Collaborations, which respectively obtained observed upper limits of 0.25 and 0.24 on the Higgs boson invisible branching fraction, $\mathcal{B}(\mathrm{H} \rightarrow$ inv $)$, at $95 \% \mathrm{CL}[39,40]$.

In the FP dark matter model [20], the DM particle, assumed to be either a Dirac or Majorana fermion, couples to a color-triplet scalar mediator $\left(\phi_{\mathrm{u}}\right)$ and an SM fermion. In the investigated model, the DM candidate is assumed to couple only to up-type quarks, with a coupling strength parameter $\lambda_{\mathrm{u}}=1$. In this model, the mediators couple to quarks and the DM candidate and may be singly produced in association with a DM particle. This associated production yields a monojet signature, while pair production of mediators can be observed in multijet final states with significant $p_{\mathrm{T}}$ imbalance, as shown in Fig. 1.

The light nonthermal DM model [21,22] is a minimal extension of the SM where the DM particle is a Majorana fermion $\left(\mathrm{n}_{\mathrm{DM}}\right)$ that interacts with the up-type quarks via a colored scalar mediator $\left(\mathrm{X}_{1}\right)$ with a coupling strength parameter $\lambda_{2}$. This new colored mediator also interacts with the down-type quarks with a coupling strength parameter $\lambda_{1}$. Baryon number is not conserved in interactions of such mediators, and therefore the nonthermal DM model could explain both the baryon abundance and the DM content of the Universe. The DM particle mass in this model must be nearly degenerate with the proton mass to ensure the stability of both the proton and the DM particle. Thus, the latter can be singly produced at the LHC, as shown in Fig. 2. This leads to a final state that includes large $p_{\mathrm{T}}$ imbalance and an energetic jet, the $p_{\mathrm{T}}$ distribution of which is a Jacobian peak at half the $\mathrm{X}_{1}$ mass.

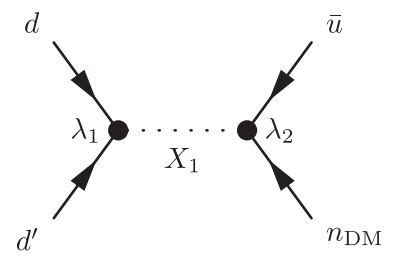

FIG. 2. Example of Feynman diagram of the main production mechanism at the LHC of DM particles in the nonthermal model resulting in the monojet final state. In this diagram, $\mathrm{d}$ and $\mathrm{d}^{\prime}$ represent different down-type quark generations.
The ADD model of EDs offers an explanation of the large difference between the electroweak unification scale and the Planck scale $\left(M_{\mathrm{Pl}}\right)$, at which gravity becomes as strong as the SM interactions. In the simplest ADD model, a number $(n)$ of EDs are introduced and are compactified on an $n$-dimensional torus of common radius $R$. In this framework, the SM particles and their interactions are confined to the ordinary $3+1$ space-time dimensions, while gravity is free to propagate through the entire multidimensional space. The strength of the gravitational force in $3+1$ dimensions is effectively diluted. The fundamental Planck scale $M_{\mathrm{D}}$ of this $4+n$-dimensional theory is related to the apparent four-dimensional Planck scale according to $M_{\mathrm{Pl}}{ }^{2} \approx M_{\mathrm{D}}{ }^{n+2} R^{n}$. The production of gravitons $(\mathrm{G})$ is expected to be greatly enhanced by the increased phase space available in the EDs. Once produced in proton-proton collisions, the graviton escapes undetected into the EDs, and its presence must be inferred from an overall $p_{\mathrm{T}}$ imbalance in the collision event, again leading to a monojet signature, as shown in Fig. 3 .

For all models, the signal extraction is performed using the distribution of the $p_{\mathrm{T}}$ imbalance in each event category. In the context of simplified DM models, the results of the search are reported in terms of excluded values of the masses of the mediator and of the DM particles. In the context of the FP and nonthermal DM models, the results of the search are reported in terms of excluded values of the mass of the mediator particle and either the DM particle mass or the strength of the coupling between the mediator and the DM or SM particles. The case of a Higgs boson decaying to invisible (e.g., DM) particles is also considered, and the results are reported in terms of upper limits on the branching fraction to invisible particles of the Higgs boson with a mass of $125 \mathrm{GeV}$ [41-43], assuming SM production cross sections $\left(\sigma_{\mathrm{SM}}\right)$. In the ADD model, the results are reported in terms of limits on the fundamental Planck scale as a function of the number of extra spatial dimensions.

This paper is organized as follows. A brief overview of the CMS detector and a description of the event reconstruction is given in Sec. II. Information about the event simulation is provided in Sec. III, and the event selection is provided in Sec. IV. Section V details the background estimation strategy used in the analysis. Finally, the results of the search are described in Sec. VI and summarized in Sec. VII. 

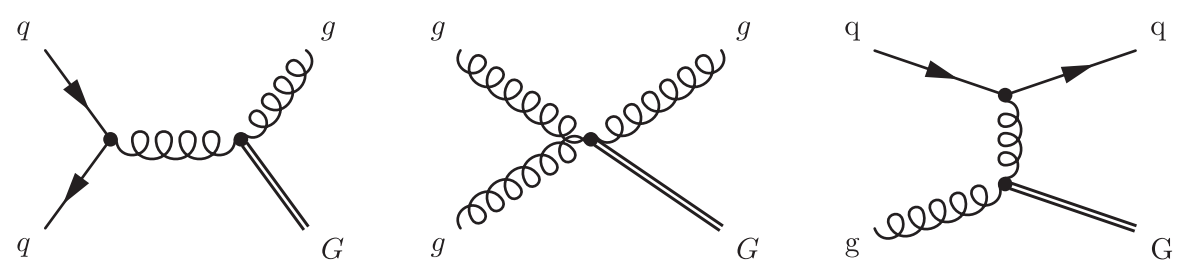

FIG. 3. Examples of Feynman diagrams of the main production mechanisms of gravitons at the LHC that provide monojet signatures in the ADD model.

\section{CMS DETECTOR AND EVENT RECONSTRUCTION}

The central feature of the CMS apparatus is a superconducting solenoid of $6 \mathrm{~m}$ internal diameter, providing a magnetic field of 3.8 T. Within the solenoid volume are a silicon pixel and strip tracker, a lead tungstate crystal electromagnetic calorimeter (ECAL), and a brass and scintillator hadron calorimeter (HCAL), each composed of a barrel and two end cap sections. Forward calorimeters extend the pseudorapidity $(\eta)$ coverage provided by the barrel and end cap detectors. Muons are detected in gasionization chambers embedded in the steel flux-return yoke outside the solenoid. A more detailed description of the CMS detector, together with a definition of the coordinate system used and the relevant kinematic variables, can be found in Ref. [44].

The CMS particle-flow (PF) event algorithm [45] reconstructs and identifies each individual particle with an optimized combination of information from the various elements of the detector. The energy of photons is directly obtained from the ECAL measurement, corrected for zerosuppression effects. The energy of muons is obtained from the curvature of the corresponding track. The energy of electrons is determined from a combination of the electron momentum at the primary interaction vertex as determined by the tracker, the energy of the corresponding ECAL cluster, and the energy sum of all bremsstrahlung photons spatially compatible with originating from the electron track. The energy of charged hadrons is determined from a combination of their momentum measured in the tracker and the matching ECAL and HCAL energy deposits, corrected for zero-suppression effects and for the response function of the calorimeters to hadronic showers. Finally, the energy of neutral hadrons is obtained from the corresponding corrected ECAL and HCAL energy.

The missing transverse momentum vector $\left(\vec{p}_{\mathrm{T}}^{\text {miss }}\right)$ is computed as the negative vector sum of the transverse momenta $\left(\vec{p}_{\mathrm{T}}\right)$ of all the PF candidates in an event, and its magnitude is denoted as $p_{\mathrm{T}}^{\text {miss }}$. Hadronic jets are reconstructed by clustering PF candidates using the infrared and collinear safe anti- $k_{\mathrm{T}}$ algorithm [46]. Jets clustered with distance parameters of 0.4 and 0.8 are referred to as AK4 and AK8 jets, respectively. The reconstructed vertex with the largest value of summed physics object $p_{\mathrm{T}}^{2}$ is taken to be the primary $p p$ interaction vertex. The physics objects are those returned by a jet finding algorithm [46,47] applied to all charged PF candidates associated with the vertex, plus the corresponding associated $p_{\mathrm{T}}^{\text {miss }}$.

Jet momentum is determined as the vector sum of all particle momenta in the jet and is found from simulation to be within $5 \%$ to $10 \%$ of the true momentum over the full $p_{\mathrm{T}}$ spectrum and detector acceptance. An offset correction is applied to jet energies to take into account the contribution from additional proton-proton interactions within the same or nearby bunch crossings (pileup). Jet energy corrections are derived from simulation and are confirmed with in situ measurements of the energy balance in dijet, multijet, $\gamma+$ jet, and leptonic $Z+$ jet events [48]. Additional selection criteria are applied to each event to remove spurious jetlike features originating from isolated noise patterns in certain HCAL regions. Such corrections and selections are also propagated to the $p_{\mathrm{T}}^{\text {miss }}$ calculation $[49,50]$.

Muons within the geometrical acceptance of $|\eta|<2.4$ are reconstructed by combining information from the silicon tracker and the muon system [51]. The muons are required to pass a set of quality criteria based on the number of spatial points measured in the tracker and in the muon system, the fit quality of the muon track, and its consistency with the primary vertex of the event. The isolation requirements for muons are based on the sum of the energies of the PF candidates originating from the primary vertex within a cone of $\Delta R<0.4$ around the muon direction, excluding the muons and electrons from the sum. The muon isolation variable is corrected for pileup effects by subtracting half of the $p_{\mathrm{T}}$ sum of the charged particles that are inside the isolation cone and not associated with the primary vertex. In this paper, "loose" muons are selected with an average efficiency of $98 \%$ and are used as a condition to veto the events, whereas "tight" muons are selected with an average efficiency of $95 \%$ and are used to tag the events in the control samples.

Electrons within the geometrical acceptance of $|\eta|<2.5$ are reconstructed by associating tracks reconstructed in the silicon detector with clusters of energy in the ECAL [52]. Well-identified electron candidates are required to satisfy additional identification criteria based on the shower shape of the energy deposit in the ECAL and the consistency of the electron track with the primary vertex [53]. Electron candidates that are identified as coming from photon conversions in the detector material are removed. The 
isolation requirements are separated from electron identification and are based on the sum of the energies of the PF candidates originating from the primary vertex within a cone of $\Delta R<0.3$ around the electron direction, excluding the muons and electrons from the sum. The mean energy deposit in the isolation cone of the electron coming from pileup is estimated following the method described in Ref. [52] and subtracted from the isolation sum. In this paper, loose electrons are selected with an average efficiency of $95 \%$ and are used as a condition to veto the events, whereas tight electrons with an average efficiency of $70 \%$ are used to select the events in the control samples.

Photon candidates are reconstructed from energy deposits in the ECAL using algorithms that constrain the clusters to the size and shape expected from a photon [54]. The identification of the candidates is based on shower-shape and isolation variables. For a photon to be considered to be isolated, scalar $p_{\mathrm{T}}$ sums of $\mathrm{PF}$ candidates originating from the primary vertex, excluding the muons and electrons within a cone of $\Delta R<0.3$ around the photon candidate, are required to be below the bounds defined. Only the PF candidates that do not overlap with the electromagnetic shower of the candidate photon are included in the isolation sums. In this paper, loose photon candidates are required to be reconstructed within $|\eta|<2.5$, whereas tight photon candidates used are required to be reconstructed in the ECAL barrel $(|\eta|<1.44)$. The tight photon candidates are also required to pass identification and isolation criteria that ensure an efficiency of $80 \%$ in selecting prompt photons and a sample purity of $95 \%$ for the control samples.

Hadronically decaying $\tau$ lepton candidates detected within $|\eta|<2.3$ are required to pass identification criteria using the hadron-plus-strips algorithm [55]. The algorithm identifies a jet as a hadronically decaying $\tau$ lepton candidate if a subset of the particles assigned to the jet is consistent with the decay products of a $\tau$ candidate. In addition, $\tau$ candidates are required to be isolated from other activity in the event. The isolation requirement is computed by summing the $p_{\mathrm{T}}$ of the PF charged and PF photon candidates within an isolation cone of $\Delta R=0.5$ and 0.3 , respectively, around the $\tau$ candidate direction. A more detailed description of the isolation requirement can be found in Ref. [55].

\section{SIMULATED SAMPLES}

To model the SM backgrounds, simulated Monte Carlo (MC) samples are produced for the $Z+$ jets, $W+$ jets, $\gamma+$ jets, and QCD multijet processes at leading order (LO) using the MADGRAPH5_aMC@NLO 2.2.2 [56] generator and are generated with up to four additional partons in the matrix element calculations. The samples for the $t \bar{t}$ and single top quark background processes are produced at nextto-leading order (NLO) using POWHEG2.0 and POWHEG1.0, respectively [57,58], and the set of diboson $(W W, W Z, Z Z)$ samples is produced at LO with PYTHIA8.205 [59].
Vector and axial-vector monojet and mono- $V$ dark matter signals are simulated at NLO using the simplified dark matter (DMSIMP) models $[60,61]$ with the MADGRAPH5_amc@ NLO generator. Both scalar and pseudoscalar monojet and mono- $\mathrm{V}$ production contain gluon-initiated loop processes. In the case of mono- $\mathrm{V}$ signals, no direct couplings of the mediator to vector bosons are considered. All samples are generated at LO with one additional parton in the matrix element calculations, taking into account finite top quark mass effects and using the MADGRAPH5_aMC@NLO generator in conjunction with the DMSIMP models.

The SM Higgs boson signal events produced through vector boson fusion and gluon fusion are generated using the POWHEG generator $[62,63]$; for each sample, the cross section is normalized to the next-to-NLO (NNLO) and next-to-NNLO, respectively. The SM Higgs boson production in association with $W$ or $Z$ bosons is simulated at $\mathrm{LO}$ using the JHUGENERATOR5.2.5 generator [64] and normalized to the NNLO cross section.

The ADD ED signal is simulated at $\mathrm{LO}$ in QCD using the PYTHIA generator, requiring $\hat{p}_{\mathrm{T}}>80 \mathrm{GeV}$, where $\hat{p}_{\mathrm{T}}$ denotes the transverse momentum of the outgoing parton in the parton-parton center-of-mass frame. The PYTHIA truncation setting is used to suppress the cross section by a factor of $M_{\mathrm{D}}{ }^{4} / \hat{s}^{2}$ for $\hat{s}>M_{\mathrm{D}}{ }^{2}$, where $\hat{s}$ is the center-ofmass energy of the incoming partons, to ensure validity of the effective field theory.

Lastly, both the FP dark matter signal and the nonthermal DM signal models are simulated at LO using the MADGRAPH5_aMC@NLO generator. In the FP dark matter signal model, the coupling strength parameter is fixed to be $\lambda_{\mathrm{u}}=1$, while in the nonthermal DM signal model, the mass of the DM particle is fixed to the proton mass to assure the stability of both the proton and the DM particle. In this latter model, coupling ranges of $0.01-1.5$ for $\lambda_{1}$ and $0.01-$ 2.0 for $\lambda_{2}$ are considered, to ensure the mediator width is less than about $30 \%$ of its mass.

The MC samples produced using MADGraPH5_ aMC@NLO, POWHEG, and JHUGENERATOR generators are interfaced with PYTHIA using the CUETP8M1 tune [65] for the fragmentation, hadronization, and underlying event description. In the case of the MADGRAPH5_aMC@NLO samples, jets from the matrix element calculations are matched to the parton shower description following the MLM [66] (FxFx [67]) prescription to match jets from matrix element calculations and the parton shower description for LO (NLO) samples. The NNPDF3.0 [68] parton distribution functions (PDFs) are used in all generated samples. The propagation of all final-state particles through the CMS detector are simulated with GEANT4 [69]. The simulated events include the effects of pileup, with the multiplicity of reconstructed primary vertices matching that in data. The average number of pileup interactions per proton bunch crossing is found to be 23 for the data sample used in this analysis [70]. 


\section{EVENT SELECTION}

Signal region events are selected using triggers with thresholds of 110 or $120 \mathrm{GeV}$ on both $p_{\mathrm{T} \text {,trig }}^{\text {mis }}$ and $H_{\mathrm{T} \text {,trig }}^{\text {miss }}$, depending on the data taking period. The $p_{\mathrm{T}, \text { trig }}^{\text {miss }}$ corresponds to the magnitude of the vector $\vec{p}_{\mathrm{T}}$ sum of all the PF candidates reconstructed at the trigger level, while the $H_{\mathrm{T}, \text { trig }}^{\text {miss }}$ is computed as the magnitude of the vector $\vec{p}_{\mathrm{T}}$ sum of jets with $p_{\mathrm{T}}>20 \mathrm{GeV}$ and $|\eta|<5.0$ reconstructed at the trigger level. The energy fraction attributed to neutral hadrons in these jets is required to be smaller than 0.9. This requirement suppresses anomalous events with jets originating from detector noise. To be able to use the same triggers for selecting events in the muon control samples used for background prediction, muon candidates are not included in the $p_{\mathrm{T} \text {,trig }}^{\text {miss }}$ nor $H_{\mathrm{T} \text {,trig }}^{\text {miss }}$ computation. The trigger efficiency is measured to be $97 \%$ for events passing the analysis selection for $p_{\mathrm{T}}^{\text {miss }}>250 \mathrm{GeV}$ and becomes fully efficient for events with $p_{\mathrm{T}}^{\text {miss }}>350 \mathrm{GeV}$.

Candidate events are required to have $p_{\mathrm{T}}^{\text {miss }}>250 \mathrm{GeV}$. In the monojet category, the highest $p_{\mathrm{T}}$ (leading) AK4 jet in the event is required to have $p_{\mathrm{T}}>100 \mathrm{GeV}$ and $|\eta|<2.4$, whereas in the mono- $V$ category, the leading AK8 jet is required to have $p_{\mathrm{T}}>250 \mathrm{GeV}$ and $|\eta|<2.4$. In both categories, the leading jet is also required to have at least $10 \%$ of its energy coming from charged particles and less than $80 \%$ of its energy attributed to neutral hadrons. This selection helps to remove events originating from beam-induced backgrounds. In addition, the analysis employs various event filters to reduce events with large misreconstructed $p_{\mathrm{T}}^{\text {miss }}$ [49] originating from noncollision backgrounds.

The main background processes in this search are the $Z(\nu \nu)+$ jets and $W(\ell \nu)+$ jets processes. The $Z(\nu \nu)+$ jets process is an irreducible background and constitutes the largest background in the search. In contrast, the background from $W(\ell \nu)+$ jets is suppressed by imposing a veto on events containing one or more loose muons or electrons with $p_{\mathrm{T}}>10 \mathrm{GeV}$, or $\tau$ leptons with $p_{\mathrm{T}}>18 \mathrm{GeV}$. Events that contain a loose, isolated photon with $p_{\mathrm{T}}>15 \mathrm{GeV}$ and $|\eta|<2.5$ are also vetoed. This helps to suppress electroweak (EW) backgrounds in which a photon is radiated from the initial state. To reduce the contamination from top quark backgrounds, events are rejected if they contain a b-tagged jet with $p_{\mathrm{T}}>20 \mathrm{GeV}$ and $|\eta|<2.4$. These jets are identified using the combined secondary vertex algorithm (CSVv2) [71,72], adopting a working point corresponding to correctly identifying a jet originating from a bottom quark with a probability of $80 \%$ and misidentifying a jet originating from a charm quark (light-flavor jet) with a probability of $40 \%$ (10)\%. Lastly, QCD multijet background with $E_{\mathrm{T}}^{\text {miss }}$ arising from mismeasurements of the jet momenta is suppressed by requiring the minimum azimuthal angle between the $\vec{p}_{\mathrm{T}}^{\text {miss }}$ direction and each of the first four leading jets with $p_{\mathrm{T}}$ greater than $30 \mathrm{GeV}$ to be larger than 0.5 radians.

To select an event in the mono- $V$ category, a leading AK8 jet is identified as a jet arising from hadronic decays of Lorentz-boosted $W$ or $Z$ bosons. Such jets typically have an invariant mass, computed from the momenta of the jet's constituents, between 65 and $105 \mathrm{GeV}$ [73]. The mass of the leading AK8 jet is computed after pruning based on the technique $[74,75]$ involving reclustering the constituents of the jet using the Cambridge-Aachen algorithm [76] and removing the soft and wide-angle contributions to jets in every recombination step. The pruning algorithm is controlled by a soft threshold parameter $\mathrm{z}_{\mathrm{cut}}=0.1$ and an angular separation threshold of $\Delta R>m_{\mathrm{jet}} / p_{\mathrm{T}}^{\mathrm{jet}}$. This technique yields improved jet mass resolution owing to reduced effects coming from the underlying event and pileup. The $N$-subjettiness variable $\tau_{N}$ [77] is also employed to further isolate jets arising from hadronic decays of $W$ or $Z$ bosons. This observable measures the distribution of jet constituents relative to candidate subjet axes in order to quantify how well the jet can be divided into $N$ subjets. Therefore, the ratio of the "2-subjettiness" to the " 1 -subjettiness" $\left(\tau_{2} / \tau_{1}\right)$ has excellent capability to distinguish jets originating from boosted vector bosons from jets originating from light quarks and gluons. The pruned jet mass and $N$ subjettiness requirements, the use of which is referred to as $V$ tagging, result in a 70\% efficiency for tagging jets originating from $V$ bosons and a $5 \%$ probability of misidentifying a jet as a $V$ jet. Events that do not qualify for the mono- $V$ category are assigned to the monojet category. The common selection requirements for both signal categories are summarized in Table I, while the category-specific selection requirements are reported in Table II.

TABLE I. Summary of the common selection requirements for mono- $V$ and monojet categories.

\begin{tabular}{lcc}
\hline \hline Variable & Selection & Target background \\
\hline Muon (electron) veto & $p_{\mathrm{T}}>10 \mathrm{GeV},|\eta|<2.4(2.5)$ & $Z(\ell \ell)+$ jets, $W(\ell \nu)+$ jets \\
$\tau$ lepton veto & $p_{\mathrm{T}}>18 \mathrm{GeV},|\eta|<2.3$ & $Z(\ell \ell)+$ jets, $W(\ell \nu)+$ jets \\
Photon veto & $p_{\mathrm{T}}>15 \mathrm{GeV},|\eta|<2.5$ & $\gamma+$ jets \\
Bottom jet veto & $\mathrm{CSVv} 2<0.8484, p_{\mathrm{T}}>15 \mathrm{GeV},|\eta|<2.4$ & Top quark \\
$p_{\mathrm{T}}^{\text {miss }}$ & $>250 \mathrm{GeV}$ & $\mathrm{QCD}$, top quark, $Z(\ell \ell)+$ jets \\
$\Delta \phi\left(\vec{p}_{\mathrm{T}}^{\text {jet }}, \vec{p}_{\mathrm{T}}^{\text {miss }}\right)$ & $>0.5$ radians & QCD \\
Leading AK4 jet $p_{\mathrm{T}}$ and $\eta$ & $>100 \mathrm{GeV}$ and $|\eta|<2.4$ & All \\
\hline \hline
\end{tabular}


TABLE II. Summary of the selection requirements for the mono- $V$ category. Events that fail the mono- $V$ selection are assigned to the monojet category.

\begin{tabular}{lc}
\hline \hline Leading AK8 jet & Mono- $V$ selection \\
\hline$p_{\mathrm{T}}$ and $\eta$ & $>250 \mathrm{GeV}$ and $|\eta|<2.4$ \\
$\tau_{2} / \tau_{1}$ & $<0.6$ \\
Mass $\left(m_{\text {jet }}\right)$ & $65<m_{\text {jet }}<105 \mathrm{GeV}$ \\
\hline \hline
\end{tabular}

\section{BACKGROUND ESTIMATION}

The largest background contributions, from $Z(\nu \nu)+$ jets and $W(\ell \nu)+$ jets processes, are estimated using data from five mutually exclusive control samples selected from dimuon, dielectron, single-muon, single-electron, and $\gamma+$ jets final states as explained below. The hadronic recoil $p_{\mathrm{T}}$ is used as a proxy for $p_{\mathrm{T}}^{\text {miss }}$ in these control samples and is defined by excluding identified leptons or photons from the $p_{\mathrm{T}}^{\text {miss }}$ calculation.

\section{A. Control sample selection}

Dimuon and single-muon control sample events are selected using full signal region criteria with the exception of the muon veto. Events in the dimuon control sample are selected requiring leading (subleading) muon $p_{\mathrm{T}}$ greater than 20 (10) GeV and an invariant mass in the range 60 to $120 \mathrm{GeV}$, compatible with a $Z$ boson decay. Events are vetoed if there is an additional loose muon or electron with $p_{\mathrm{T}}>10 \mathrm{GeV}$. In the single-muon control sample, exactly one tightly identified, isolated muon with $p_{\mathrm{T}}>$ $20 \mathrm{GeV}$ is required. No additional loose muons or electrons with $p_{\mathrm{T}}>10 \mathrm{GeV}$ are allowed. In addition, the transverse mass $\left(M_{\mathrm{T}}\right)$ of the muon- $\vec{p}_{\mathrm{T}}^{\text {miss }}$ system is required to be less than $160 \mathrm{GeV}$ and is computed as $M_{\mathrm{T}}=\sqrt{2 p_{\mathrm{T}}^{\text {miss }} p_{\mathrm{T}}^{\mu}(1-\cos \Delta \phi)}$, where $p_{\mathrm{T}}^{\mu}$ is the $p_{\mathrm{T}}$ of the muon and $\Delta \phi$ is the angle between $\vec{p}_{\mathrm{T}}^{\mu}$ and $\vec{p}_{\mathrm{T}}^{\text {miss }}$.

Dielectron and single-electron control sample events are selected with an isolated single-electron trigger with a $p_{\mathrm{T}}$ threshold of $27 \mathrm{GeV}$. In boosted $Z(e e)+$ jets events, the two electrons produced in the decay typically have so little separation such that their tracks are included in each other's isolation cones. Therefore, to recover efficiency in selecting high- $p_{\mathrm{T}} Z$ candidates at the trigger level, a nonisolated single-electron trigger with a $p_{\mathrm{T}}$ threshold of $105 \mathrm{GeV}$ is used. Events in the dielectron control sample are required to contain exactly two oppositely charged electrons with leading (trailing) electron $p_{\mathrm{T}}$ greater than 40 (10) GeV. Similar to the dimuon control sample case, the invariant mass of the dielectron system is required to be between 60 and $120 \mathrm{GeV}$ to be consistent with a $Z$ boson decay. The events in the single-electron control sample are required to contain exactly one tightly identified and isolated electron with $p_{\mathrm{T}}>40 \mathrm{GeV}$. In addition, the contamination from QCD multijet events in this control sample is suppressed by requiring $p_{\mathrm{T}}^{\text {miss }}>50 \mathrm{GeV}$ and $M_{\mathrm{T}}<160 \mathrm{GeV}$.

Lastly, the $\gamma+$ jets control sample is selected using events with one high- $p_{\mathrm{T}}$ photon collected using singlephoton triggers with $p_{\mathrm{T}}$ thresholds of 165 or $175 \mathrm{GeV}$, depending on the data taking conditions. The photon is required to have $p_{\mathrm{T}}>175 \mathrm{GeV}$ and to pass tight identification and isolation criteria, to ensure a high trigger efficiency of $98 \%$.

TABLE III. Theoretical uncertainties considered in the $V$-jets and $\gamma+$ jets processes, and their ratios. The correlation between each process and between the $p_{\mathrm{T}}$ bins are described.

\begin{tabular}{lll}
\hline \hline Uncertainty source & \multicolumn{1}{c}{ Process (magnitude) } & Correlation \\
\hline Factorization and renormalization scales (QCD) & $Z \rightarrow \nu \nu / W \rightarrow \ell \nu(0.1-0.5 \%)$ & Correlated between processes; \\
& $Z \rightarrow \nu \nu / \gamma+$ jets $(0.2-0.5 \%)$ & and in $p_{\mathrm{T}}$ \\
$p_{\mathrm{T}}$-shape dependence (QCD) & $Z \rightarrow \nu \nu / W \rightarrow \ell \nu(0.4-0.1 \%)$ & Correlated between processes; \\
& $Z \rightarrow \nu \nu / \gamma+$ jets $(0.1-0.2 \%)$ & and in $p_{\mathrm{T}}$ \\
Process dependence (QCD) & $Z \rightarrow \nu \nu / W \rightarrow \ell \nu(0.4-1.5 \%)$ & Correlated between processes; \\
& $Z \rightarrow \nu \nu / \gamma+$ jets $(1.5-3.0 \%)$ & and in $p_{\mathrm{T}}$ \\
Effects of unknown Sudakov logs (EW) & $Z \rightarrow \nu \nu / W \rightarrow \ell \nu(0-0.5 \%)$ & Correlated between processes; \\
& $Z \rightarrow \nu \nu / \gamma+$ jets $(0.1-1.5 \%)$ & and in $p_{\mathrm{T}}$ \\
Missing NNLO effects (EW) & $Z \rightarrow \nu \nu(0.2-3.0 \%)$ & Uncorrelated between processes; \\
& $\gamma+$ jets $(0.1-1.0 \%)$ & correlated in $p_{\mathrm{T}}$ \\
Effects of NLL Sudakov approx. (EW) & $W \rightarrow \ell \nu(0.4-4.5 \%)$ & Uncorrelated between processes; \\
& $Z \rightarrow \nu \nu(0.2-4.0 \%)$ & correlated in $p_{\mathrm{T}}$ \\
Unfactorized mixed QCD-EW corrections & $W \rightarrow \ell \nu(0-1.0 \%)$ & Correlated between processes; \\
PDF & $\gamma+$ jets $(0.1-3.0 \%)$ & and in $p_{\mathrm{T}}$ \\
& $Z \rightarrow \nu \nu / W \rightarrow \ell \nu(0.15-0.3 \%)$ & Correlated between processes; \\
\hline \hline
\end{tabular}



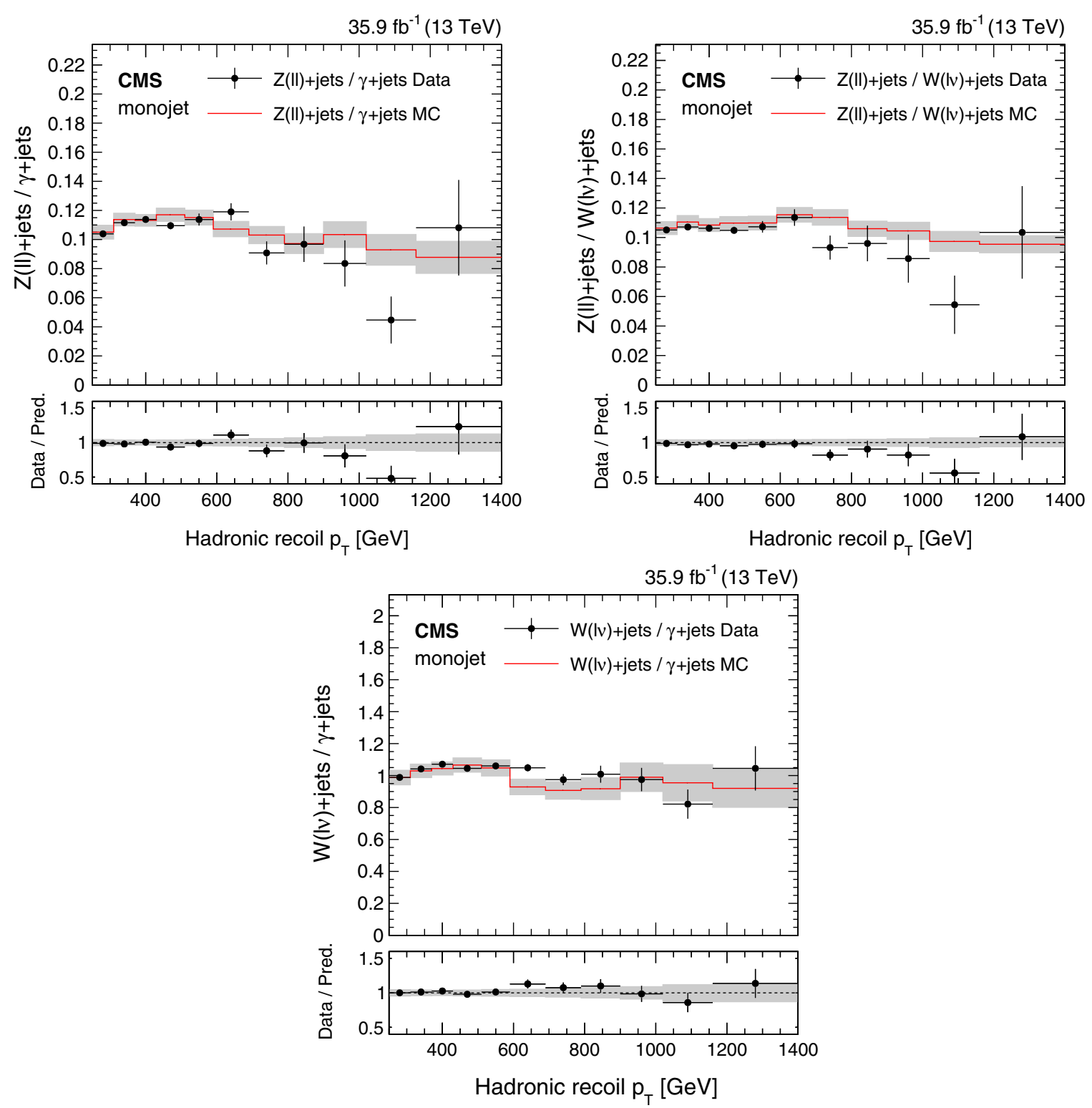

FIG. 4. Comparison between data and MC simulation for the $Z(\ell \ell) / \gamma+$ jets, $Z(\ell \ell) / W(\ell \nu)$, and $W(\ell \nu) / \gamma+$ jets ratios as a function of the hadronic recoil in the monojet category. In the lower panels, ratios of data with the prefit background prediction are shown. The gray bands include both the prefit systematic uncertainties and the statistical uncertainty in the simulation.

\section{B. Signal extraction}

A binned likelihood fit to the data as presented in Ref. [14] is performed simultaneously in the five different control samples and in the signal region, for events selected in both the monojet and mono- $V$ categories, to estimate the $Z(\nu \nu)+$ jets and $W(\ell \nu)+$ jets rate in each $p_{\mathrm{T}}^{\text {miss }}$ bin. In this likelihood, the expected numbers of $Z(\nu \nu)+$ jets events in each bin of $p_{\mathrm{T}}^{\mathrm{miss}}$ are the free parameters of the fit. Transfer factors, derived from simulation, are used to link the yields of the $Z(\ell \ell)+$ jets, $W(\ell \nu)+$ jets and $\gamma+$ jets processes in the control regions with the $Z(\nu \nu)+$ jets and $W(\ell \nu)+$ jets background estimates in the signal region. These transfer factors are defined as the ratio of expected yields of the target process in the signal region and the process being measured in the control sample.

To estimate the $W(\ell \nu)+$ jets background in the signal region, the transfer factors are constructed using the event yields of the $W(\mu \nu)+$ jets and $W(e \nu)+$ jets processes in the single-lepton control samples and the $W(\ell \nu)+$ jets process in the signal region. These transfer factors take into account the impact of lepton acceptances and efficiencies, lepton veto efficiencies, and the difference in the trigger efficiencies in the case of the single-electron control sample.

The $Z \rightarrow \nu \nu$ background prediction in the signal region is connected to the yields of $Z \rightarrow \mu^{+} \mu^{-}$and $Z \rightarrow e^{+} e^{-}$ 

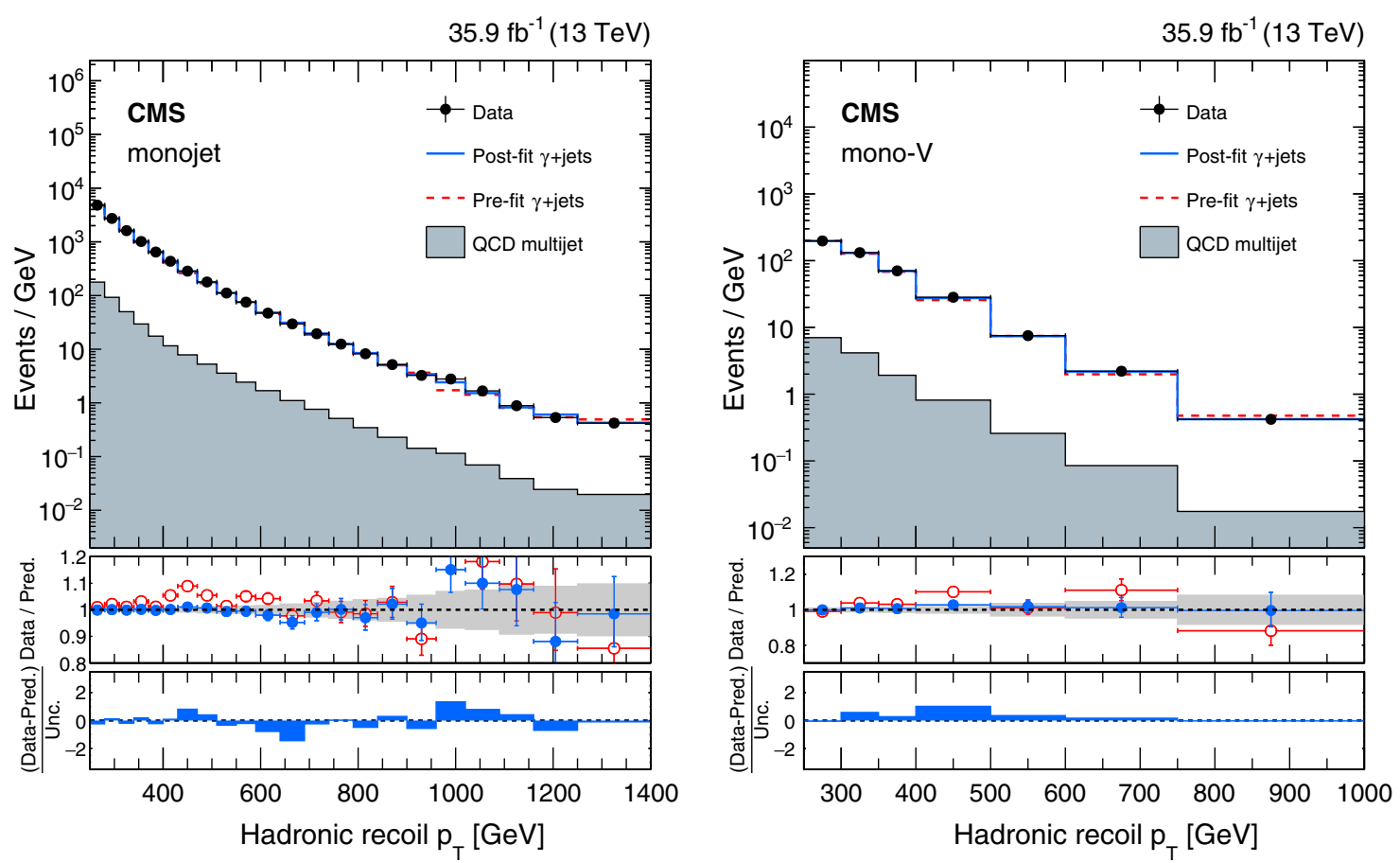

FIG. 5. Comparison between data and MC simulation in the $\gamma+$ jets control sample before and after performing the simultaneous fit across all the control samples and the signal region assuming the absence of any signal. The left plot shows the monojet category, and the right plot shows the mono- $V$ category. The hadronic recoil $p_{\mathrm{T}}$ in $\gamma+$ jets events is used as a proxy for $p_{\mathrm{T}}^{\text {miss }}$ in the signal region. The last bin includes all events with hadronic recoil $p_{\mathrm{T}}$ larger than 1250 (750) GeV in the monojet (mono- $V$ ) category. In the lower panels, ratios of data with the prefit background prediction (red open points) and postfit background prediction (blue full points) are shown for both the monojet and mono- $V$ categories. The gray band in the lower panel indicates the postfit uncertainty after combining all the systematic uncertainties. Finally, the distribution of the pulls, defined as the difference between data and the postfit background prediction relative to the quadrature sum of the postfit uncertainty in the prediction and statistical uncertainty in data, is shown in the lowest panel.

events in the dilepton control samples. The associated transfer factors account for the differences in the branching ratio of $Z$ bosons to charged leptons relative to neutrinos and the impact of lepton acceptance and selection efficiencies. In the case of dielectron events, the transfer factor also takes into account the difference in the trigger efficiencies. The resulting constraint on the $Z(\nu \nu)+$ jets process from the dilepton control samples is limited by the statistical uncertainty in the dilepton control samples because of the large difference in branching fractions between $Z$ boson decays to neutrinos and $Z$ boson decays to muons and electrons.

The $\gamma+$ jets control sample is also used to predict the $Z(\nu \nu)+$ jets process in the signal region through a transfer factor, which accounts for the difference in the cross sections of the $\gamma+$ jets and $Z(\nu \nu)+$ jets processes, the effect of acceptance and efficiency of identifying photons along with the difference in the efficiencies of the photon and $p_{\mathrm{T}}^{\text {miss }}$ triggers. The addition of the $\gamma+$ jets control sample mitigates the impact of the limited statistical power of the dilepton constraint, because of the larger production cross section of $\gamma+$ jets process compared to that of $Z(\nu \nu)+$ jets process.
Finally, a transfer factor is also defined to connect the $Z(\nu \nu)+$ jets and $W(\ell \nu)+$ jets background yields in the signal region, to further benefit from the larger statistical power that the $W(\ell \nu)+$ jets background provides, making it possible to experimentally constrain $Z(\nu \nu)+$ jets production at high $p_{\mathrm{T}}^{\text {miss }}$.

These transfer factors rely on an accurate prediction of the ratio of $Z+$ jets, $W+$ jets, and $\gamma+$ jets cross sections. Therefore, $\mathrm{LO}$ simulations for these processes are corrected using boson $p_{\mathrm{T}}$-dependent NLO QCD K-factors derived using MADGrAPH5_aMC@NLO. They are also corrected using

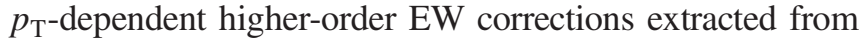
theoretical calculations [78-83]. The higher-order corrections are found to improve the data-to-simulation agreement for both the absolute prediction of the individual $Z+$ jets, $W+$ jets, and $\gamma+$ jets processes and their respective ratios.

The remaining backgrounds that contribute to the total event yield in the signal region are much smaller than those from $Z(\nu \nu)+$ jets and $W(\ell \nu)+$ jets processes. These smaller backgrounds include QCD multijet events which are measured from data using a $\Delta \phi$ extrapolation method $[14,84]$ and top quark and diboson processes, which are obtained directly from simulation. 

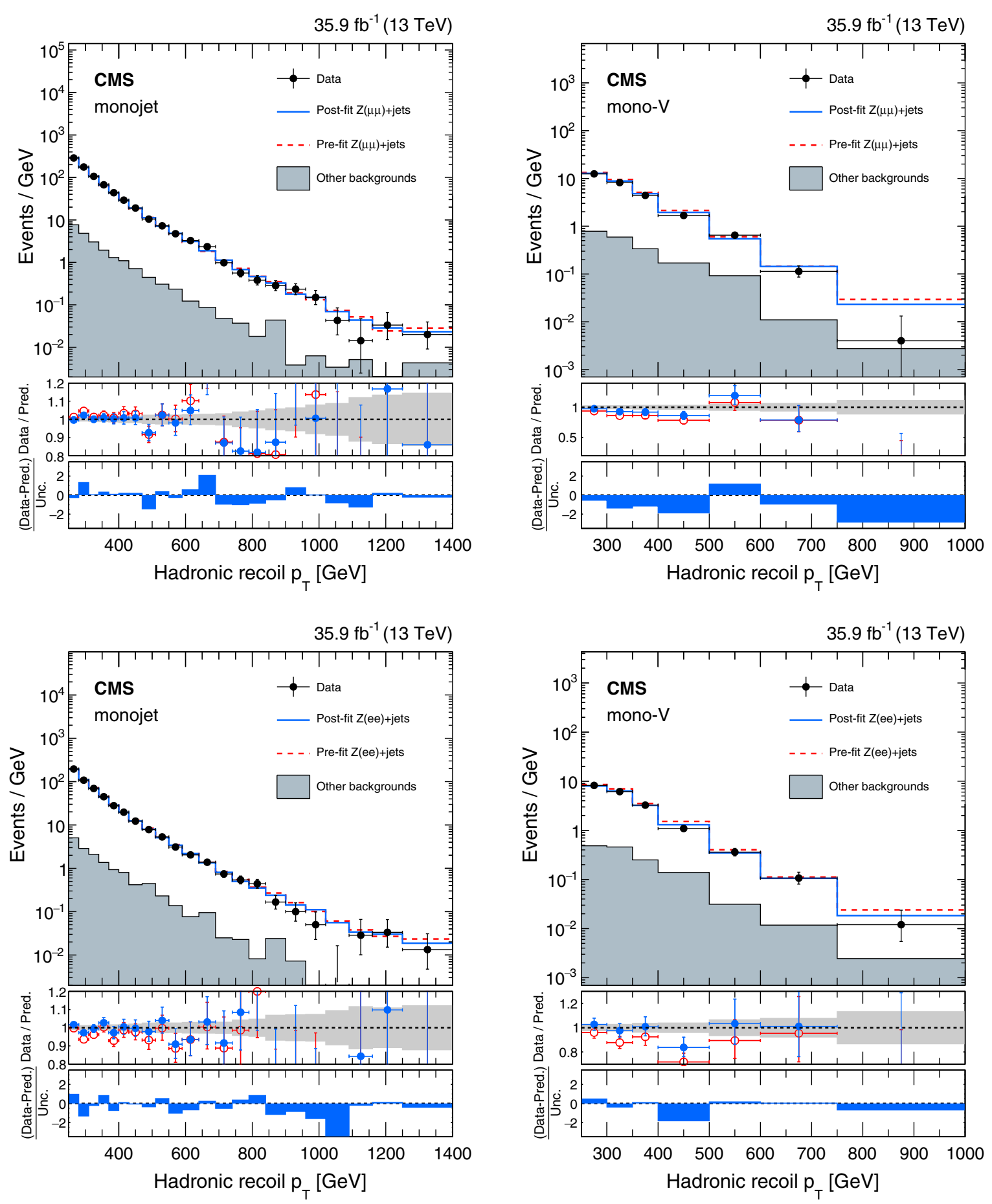

FIG. 6. Comparison between data and MC simulation in the dimuon (upper row) and dielectron (lower row) control samples before and after performing the simultaneous fit across all the control samples and the signal region assuming the absence of any signal. Plots correspond to the monojet (left) and mono- $V$ (right) categories, respectively, in the dilepton control sample. The hadronic recoil $p_{\mathrm{T}}$ in dilepton events is used as a proxy for $p_{\mathrm{T}}^{\text {miss }}$ in the signal region. The other backgrounds include top quark, diboson, and $W+$ jets processes. The description of the lower panels is the same as in Fig. 5.

\section{Systematic uncertainties}

Systematic uncertainties in the transfer factors are modeled as constrained nuisance parameters and include both experimental and theoretical uncertainties in the $\gamma+$ jets to $Z+$ jets and $W+$ jets to $Z+$ jets differential cross section ratios.
Theoretical uncertainties in $V$-jets and $\gamma+$ jets processes include effects from QCD and EW higher-order corrections along with PDF modeling uncertainty. To estimate the theoretical uncertainty in the $V$-jets and $\gamma+$ jets ratios due to QCD and EW higher-order effects as well as their 

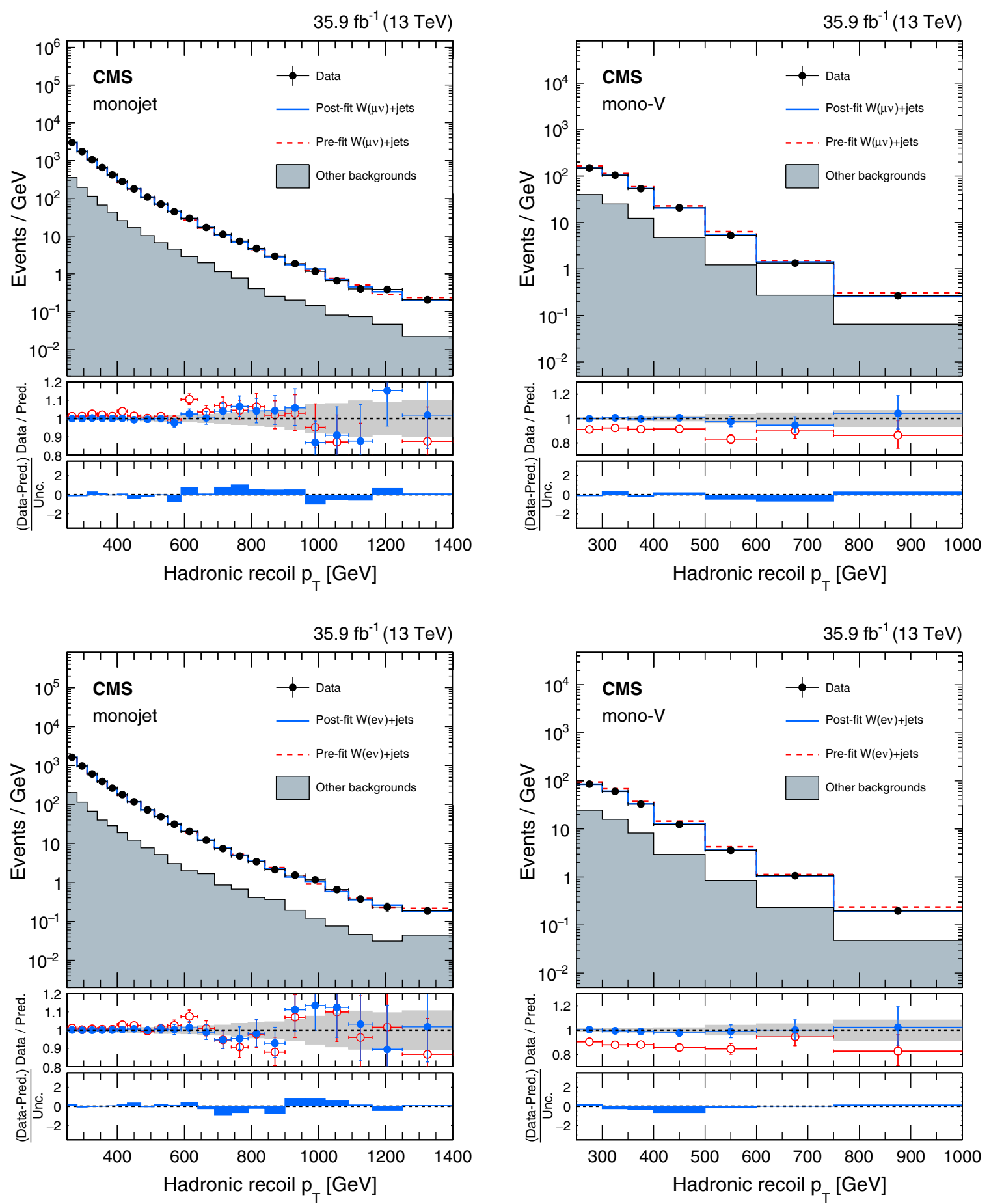

FIG. 7. Comparison between data and MC simulation in the single-muon (upper row) and single-electron (lower row) control samples before and after performing the simultaneous fit across all the control samples and the signal region assuming the absence of any signal. Plots correspond to the monojet (left) and mono- $V$ (right) categories, respectively, in the single-lepton control samples. The hadronic recoil $p_{\mathrm{T}}$ in single-lepton events is used as a proxy for $p_{\mathrm{T}}^{\text {miss }}$ in the signal region. The other backgrounds include top quark, diboson, and QCD multijet processes. The description of the lower panels is the same as in Fig. 5.

correlations across the processes and $p_{\mathrm{T}}$ bins, the recommendations of Ref. [16] are employed, as detailed in the following explanation.

Three separate sources of uncertainty associated with QCD higher-order corrections are used. One of the uncertainties considered comes from the variations around the central renormalization and factorization scale choice. It is evaluated by taking the differences in the NLO cross section as a function of boson $p_{\mathrm{T}}$ after changing the renormalization and factorization scales by a factor of 2 
and a factor of $1 / 2$ with respect to the default value. These constant scale variations mainly affect the overall normalization of the boson $p_{\mathrm{T}}$ distributions and therefore underestimate the shape uncertainties that play an important role in the extrapolation of low- $p_{\mathrm{T}}$ measurements to high $p_{\mathrm{T}}$. A second, conservative shape uncertainty derived from altered boson $p_{\mathrm{T}}$ spectra is used to supplement the scale uncertainties and account for the $p_{\mathrm{T}}$ dependence of the uncertainties. The modeling of the correlations between the processes assumes a close similarity of QCD effects between all $V$-jets and $\gamma+$ jets processes. However, the QCD effects in $\gamma+$ jets production could differ compared to the case of $Z+$ jets and $W+$ jets productions. In order to account for this variation, a third uncertainty is computed based on the difference of the known QCD K-factors of the $W+$ jets and $\gamma+$ jets processes with respect to $Z+$ jets production. All QCD uncertainties are correlated across the $Z+$ jets, $W+$ jets, and $\gamma+$ jets processes and also correlated across the bins of the hadronic recoil $p_{\mathrm{T}}$.

For the $V$-jets and $\gamma+$ jets processes, nNLO EW corrections are applied, which correspond to full NLO EW corrections [78-80,83] supplemented by two-loop Sudakov EW logarithms [81,85-87]. We also considered three separate sources of uncertainty arising from the following: pure EW higher-order corrections failing to cover the effects of unknown Sudakov logarithms in the perturbative expansion beyond NNLO, missing NNLO effects that are not included in the nNLO EW calculations, and the difference between the next-to-leading logarithmic (NLL) Sudakov approximation at two-loop and simple exponentiation of the full NLO EW correction. The variations due to the effect of unknown Sudakov logs are correlated across the $Z+$ jets, $W+$ jets, and $\gamma+$ jets processes and are also correlated across the bins of hadronic recoil $p_{\mathrm{T}}$. On the other hand, the other two sources of EW uncertainties are treated as uncorrelated across the $V$-jet and $\gamma+$ jets processes, and an independent nuisance parameter is used for each process.

A recommendation that includes a factorized approach to partially include mixed QCD-EW corrections is outlined in Ref. [16]. An additional uncertainty is introduced to account for the difference between the corrections done in the multiplicative and the additive approaches, to account for the nonfactorized mixed EW-QCD effects.

The summary of the aforementioned theoretical uncertainties including their magnitude and correlation is outlined in Table III.

Experimental uncertainties including the reconstruction efficiency (1\% per muon or electron) and the selection efficiencies of leptons (1\% per muon and $2 \%$ per electron), photons (2\%), and hadronically decaying $\tau$ leptons (5\%) are also incorporated. These reconstruction and selection efficiencies further translate into an uncertainty in the lepton veto efficiency of $3 \%$. Uncertainties in the purity
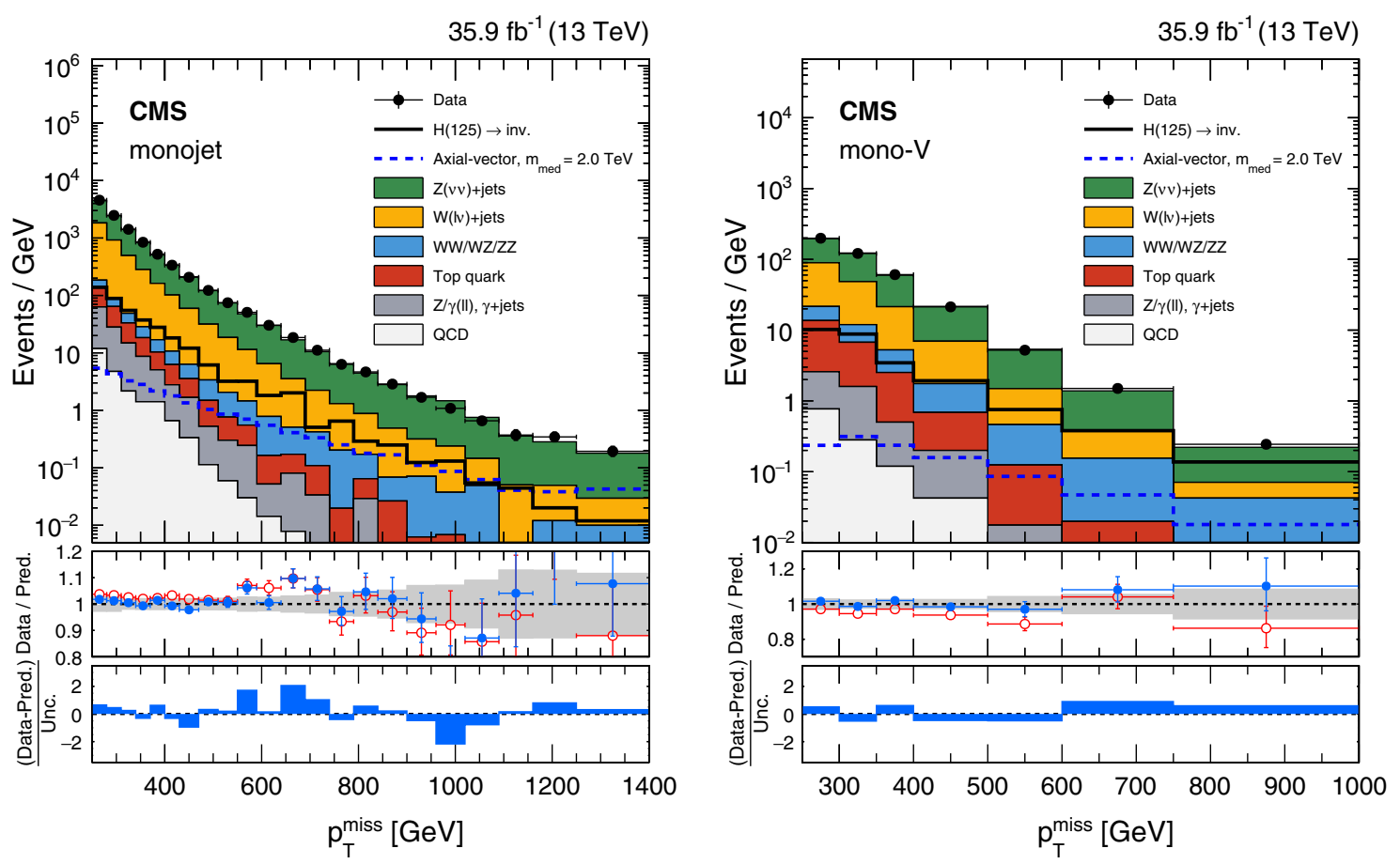

FIG. 8. Observed $p_{\mathrm{T}}^{\text {miss }}$ distribution in the monojet (left) and mono- $V$ (right) signal regions compared with the postfit background expectations for various SM processes. The last bin includes all events with $p_{\mathrm{T}}^{\text {miss }}>1250(750) \mathrm{GeV}$ for the monojet (mono- $V$ ) category. The expected background distributions are evaluated after performing a combined fit to the data in all the control samples, not including the signal region. Expected signal distributions for the $125 \mathrm{GeV}$ Higgs boson decaying exclusively to invisible particles and a $2 \mathrm{TeV}$ axial-vector mediator decaying to $1 \mathrm{GeV}$ DM particles are overlaid. The description of the lower panels is the same as in Fig. 5 . 
of photons in the $\gamma+$ jets control sample (2\%), and in the efficiency of the electron (2\%), photon (2\%), and $p_{\mathrm{T}}^{\text {miss }}$ $(1 \%-4 \%)$ triggers, are included and are fully correlated across all the bins of hadronic recoil $p_{\mathrm{T}}$ and $p_{\mathrm{T}}^{\text {miss }}$. The uncertainty in the efficiency of the $b$ jet veto is estimated to be $6 \%$ (2\%) for the contribution of the top quark (diboson) background.

The uncertainty in the efficiency of the $V$ tagging requirements is estimated to be $9 \%$ in the mono- $V$ category. The uncertainty in the modeling of $p_{\mathrm{T}}^{\text {miss }}$ in simulation [50] is estimated to be $4 \%$ and is dominated by the uncertainty in the jet energy scale.

A systematic uncertainty of $10 \%$ is included for the top quark background associated with the modeling of the top quark $p_{\mathrm{T}}$ distribution in simulation [88]. In addition, systematic uncertainties of $10 \%$ and $20 \%$ are included in the normalizations of the top quark [89] and diboson backgrounds [90,91], respectively, to account for the uncertainties in their cross sections in the relevant kinematic phase space. Lastly, the uncertainty in the QCD multijet background estimate is found to be between 50\% and $150 \%$ due to the variations of the jet response and the statistical uncertainty of the extrapolation factors.

\section{Control sample validation}

An important cross-check of the application of $p_{\mathrm{T}^{-}}$ dependent NLO QCD and EW corrections is represented by the agreement between data and simulation in the ratio of $Z+$ jets events to both $\gamma+$ jets events and $W+$ jets events in the control samples, as a function of hadronic recoil $p_{\mathrm{T}}$.

Figure 4 shows the ratio between $Z(\ell \ell)+$ jets and $\gamma+$ jets (left), $Z(\ell \ell)+$ jets and $W(\ell \nu)+$ jets (middle), and the one between the $W(\ell \nu)+$ jets $/ \gamma+$ jets processes (right) as a function of the recoil for events selected in the monojet category. While we do not explicitly use a $W(\ell \nu)+$ jets $/ \gamma+$ jets constraint in the analysis, the two cross sections are connected through the $Z+$ jets $/ \gamma+$ jets and $Z+$ jets $/ W+$ jets constraints that are explained in Sec. $V$ B. Therefore, it is instructive to examine the data-MC comparison of the $W(\ell \nu)+$ jets $/ \gamma+$ jets ratio. Good agreement is observed between data and simulation after the application of the NLO corrections as shown in Fig. 4. The ratio between $Z(\mu \mu)+$ jets and $\gamma+$ jets, $Z(\mu \mu)+$ jets and $W(\mu \nu)+$ jets, and the one between $W(\mu \nu)+$ jets $/ \gamma+$ jets processes as a function of the boson $p_{\mathrm{T}}$ are also studied, and the results can be seen in Fig. 19.

Figures 5-7 show the results of the combined fit in all control samples and the signal region. Data in the control samples are compared to the prefit predictions from simulation and the postfit estimates obtained after performing the fit. The control samples with larger yields dominate the fit results. A normalization difference of $7 \%$ is observed in the prefit distributions for the mono- $\mathrm{V}$ category in the

TABLE IV. Expected event yields in each $p_{\mathrm{T}}^{\text {miss }}$ bin for various background processes in the monojet signal region. The background yields and the corresponding uncertainties are obtained after performing a combined fit to data in all the control samples, excluding data in the signal region. The other backgrounds include QCD multijet and $\gamma+$ jets processes. The expected signal contribution for a $2 \mathrm{TeV}$ axial-vector mediator decaying to $1 \mathrm{GeV} \mathrm{DM}$ particles and the observed event yields in the monojet signal region are also reported.

\begin{tabular}{|c|c|c|c|c|c|c|c|c|}
\hline$p_{\mathrm{T}}^{\text {miss }}(\mathrm{GeV})$ & Signal & $Z(\nu \nu)+$ jets & $W(\ell \nu)+$ jets & Top quark & Diboson & Other & Total background & Data \\
\hline $250-280$ & $162 \pm 3$ & $79700 \pm 2300$ & $49200 \pm 1400$ & $2360 \pm 200$ & $1380 \pm 220$ & $1890 \pm 240$ & $134500 \pm 3700$ & 136865 \\
\hline $280-310$ & $130 \pm 3$ & $45800 \pm 1300$ & $24950 \pm 730$ & $1184 \pm 99$ & $770 \pm 120$ & $840 \pm 110$ & $73400 \pm 2000$ & 74340 \\
\hline $310-340$ & $97.8 \pm 2.4$ & $27480 \pm 560$ & $13380 \pm 260$ & $551 \pm 53$ & $469 \pm 77$ & $445 \pm 63$ & $42320 \pm 810$ & 42540 \\
\hline $340-370$ & $84.8 \pm 2.1$ & $17020 \pm 350$ & $7610 \pm 150$ & $292 \pm 28$ & $301 \pm 51$ & $260 \pm 39$ & $25490 \pm 490$ & 25316 \\
\hline $370-400$ & $65.2 \pm 1.9$ & $10560 \pm 220$ & $4361 \pm 91$ & $157 \pm 17$ & $198 \pm 33$ & $152 \pm 26$ & $15430 \pm 310$ & 15653 \\
\hline $400-430$ & $53.5 \pm 1.8$ & $7110 \pm 130$ & $2730 \pm 47$ & $104 \pm 12$ & $133 \pm 23$ & $84 \pm 15$ & $10160 \pm 170$ & 10092 \\
\hline $430-470$ & $53.9 \pm 1.8$ & $6110 \pm 100$ & $2123 \pm 37$ & $75.2 \pm 7.9$ & $110 \pm 19$ & $67 \pm 11$ & $8480 \pm 140$ & 8298 \\
\hline $470-510$ & $41.4 \pm 1.5$ & $3601 \pm 75$ & $1128 \pm 22$ & $38.6 \pm 5.3$ & $75 \pm 12$ & $21.0 \pm 3.9$ & $4865 \pm 95$ & 4906 \\
\hline $510-550$ & $34.3 \pm 1.4$ & $2229 \pm 39$ & $658 \pm 12$ & $18.5 \pm 3.3$ & $51.7 \pm 9.5$ & $12 \pm 2.4$ & $2970 \pm 49$ & 2987 \\
\hline $550-590$ & $28.1 \pm 1.2$ & $1458 \pm 27$ & $398 \pm 8$ & $12.3 \pm 2.6$ & $35.9 \pm 7.1$ & $9.7 \pm 1.9$ & $1915 \pm 33$ & 2032 \\
\hline $590-640$ & $27.5 \pm 1.2$ & $1182 \pm 26$ & $284 \pm 7$ & $5.5 \pm 1.4$ & $30.9 \pm 5.7$ & $2.6 \pm 0.7$ & $1506 \pm 32$ & 1514 \\
\hline 640-690 & $20.4 \pm 1.1$ & $667 \pm 15$ & $151 \pm 4$ & $4.6 \pm 1.7$ & $16.7 \pm 3.9$ & $4.0 \pm 0.8$ & $844 \pm 18$ & 926 \\
\hline $690-740$ & $16.6 \pm 0.9$ & $415 \pm 12$ & $90.4 \pm 3.0$ & $3.8 \pm 1.5$ & $15.6 \pm 3.6$ & $1.7 \pm 0.4$ & $526 \pm 14$ & 557 \\
\hline 740-790 & $12.5 \pm 0.8$ & $259 \pm 9.6$ & $55.2 \pm 2.3$ & $0.8 \pm 0.5$ & $9.14 \pm 2.3$ & $0.2 \pm 0.1$ & $325 \pm 12$ & 316 \\
\hline 790-840 & $8.94 \pm 0.72$ & $178 \pm 7.1$ & $35.3 \pm 1.7$ & $1.7 \pm 0.8$ & $5.35 \pm 1.7$ & $1.4 \pm 0.3$ & $223 \pm 9$ & 233 \\
\hline $840-900$ & $10.1 \pm 0.7$ & $139 \pm 6.2$ & $25.2 \pm 1.3$ & $1.5 \pm 1.2$ & $2.52 \pm 1.05$ & $0.04 \pm 0.03$ & $169 \pm 8$ & 172 \\
\hline 900-960 & $6.62 \pm 0.61$ & $88.1 \pm 4.9$ & $14.7 \pm 0.9$ & $0.3 \pm 0.3$ & $3.88 \pm 1.42$ & $0.03 \pm 0.02$ & $107 \pm 6$ & 101 \\
\hline 960-1020 & $5.19 \pm 0.54$ & $73.8 \pm 4.7$ & $12.0 \pm 0.8$ & $0.4 \pm 0.3$ & $1.83 \pm 0.92$ & $0.02 \pm 0.01$ & $88.1 \pm 5.3$ & 65 \\
\hline 1020-1090 & $4.35 \pm 0.52$ & $42.6 \pm 3.1$ & $6.7 \pm 0.6$ & $0.0 \pm 0.0$ & $3.42 \pm 1.33$ & $0.01 \pm 0.01$ & $52.8 \pm 3.9$ & 46 \\
\hline 1090-1160 & $2.84 \pm 0.43$ & $21.5 \pm 2.1$ & $3.5 \pm 0.4$ & $0.0 \pm 0.0$ & $0.00 \pm 0.00$ & $0.01 \pm 0.00$ & $25.0 \pm 2.5$ & 26 \\
\hline $1160-1250$ & $3.44 \pm 0.38$ & $21.0 \pm 2.2$ & $3.3 \pm 0.4$ & $0.0 \pm 0.0$ & $1.07 \pm 0.69$ & $0.01 \pm 0.00$ & $25.5 \pm 2.6$ & 31 \\
\hline$>1250$ & $6.39 \pm 0.58$ & $22.5 \pm 2.4$ & $2.9 \pm 0.3$ & $0.0 \pm 0.0$ & $1.49 \pm 0.91$ & $0.01 \pm 0.00$ & $26.9 \pm 2.8$ & 29 \\
\hline
\end{tabular}


TABLE V. Expected event yields in each $p_{\mathrm{T}}^{\text {miss }}$ bin for various background processes in the mono- $V$ signal region. The background yields and the corresponding uncertainties are obtained after performing a combined fit to data in all the control samples but excluding data in the signal region. The other backgrounds include QCD multijet and $\gamma+$ jets processes. The expected signal contribution for a $2 \mathrm{TeV}$ axial-vector mediator decaying to $1 \mathrm{GeV} \mathrm{DM}$ particles and the observed event yields in the mono- $V$ signal region are also reported.

\begin{tabular}{lccccccrr}
\hline \hline$p_{\mathrm{T}}^{\text {miss }}(\mathrm{GeV})$ & Signal & $Z(\nu \nu)+$ jets & $W(\ell \nu)+$ jets & Top quark & Diboson & Other & Total background & Data \\
\hline $250-300$ & $11.7 \pm 0.6$ & $5300 \pm 170$ & $3390 \pm 120$ & $553 \pm 54$ & $396 \pm 69$ & $128 \pm 25$ & $9770 \pm 290$ & 9929 \\
$300-350$ & $15.7 \pm 0.7$ & $3720 \pm 98$ & $1823 \pm 53$ & $257 \pm 27$ & $261 \pm 46$ & $79.8 \pm 13$ & $6140 \pm 140$ & 6057 \\
$350-400$ & $11.8 \pm 0.6$ & $1911 \pm 59$ & $808 \pm 28$ & $101 \pm 12$ & $134 \pm 25$ & $25.0 \pm 4.8$ & $2982 \pm 79$ & 3041 \\
$400-500$ & $15.8 \pm 0.7$ & $1468 \pm 45$ & $521 \pm 15$ & $48.8 \pm 5.7$ & $107 \pm 20$ & $20.0 \pm 3.6$ & $2165 \pm 55$ & 2131 \\
$500-600$ & $8.59 \pm 0.56$ & $388 \pm 18$ & $103.0 \pm 5.1$ & $10.7 \pm 1.9$ & $33.8 \pm 7.0$ & $1.76 \pm 0.53$ & $537 \pm 23$ & 521 \\
$600-750$ & $7.04 \pm 0.47$ & $151.0 \pm 9.9$ & $33.4 \pm 2.3$ & $1.9 \pm 1.1$ & $20.2 \pm 4.5$ & $1.05 \pm 0.25$ & $208 \pm 11$ & 225 \\
$>750$ & $4.48 \pm 0.40$ & $37.7 \pm 3.7$ & $7.09 \pm 0.69$ & $0.28 \pm 0.25$ & $10.2 \pm 2.3$ & $0.06 \pm 0.03$ & $55.3 \pm 4.6$ & 61 \\
\hline \hline
\end{tabular}

single-lepton and dilepton control regions. The sources of the differences are identified to be the modeling of the pruned mass variable and the large theoretical uncertainties in the diboson and top quark backgrounds, which are the leading backgrounds in these regions. The normalization difference is found to be fully mitigated by the fitting procedure.

\section{RESULTS AND INTERPRETATION}

The search is performed by extracting the signal through a combined fit of the signal and control regions. Figure 8 shows the comparison between data and the postfit background predictions in the signal region in the monojet and mono- $V$ categories, where the background prediction is obtained from a combined fit performed in all control regions, excluding the signal region. Expected signal distributions for the $125 \mathrm{GeV}$ Higgs boson decaying exclusively to invisible particles and a $2 \mathrm{TeV}$ axial-vector mediator decaying to $1 \mathrm{GeV}$ DM particles are overlaid. Data are found to be in agreement with the SM prediction.

The expected yields in each bin of $p_{\mathrm{T}}^{\text {miss }}$ for all SM backgrounds, after the fit to the data in the control regions, are given in Tables IV and $\mathrm{V}$ for the monojet and mono- $V$
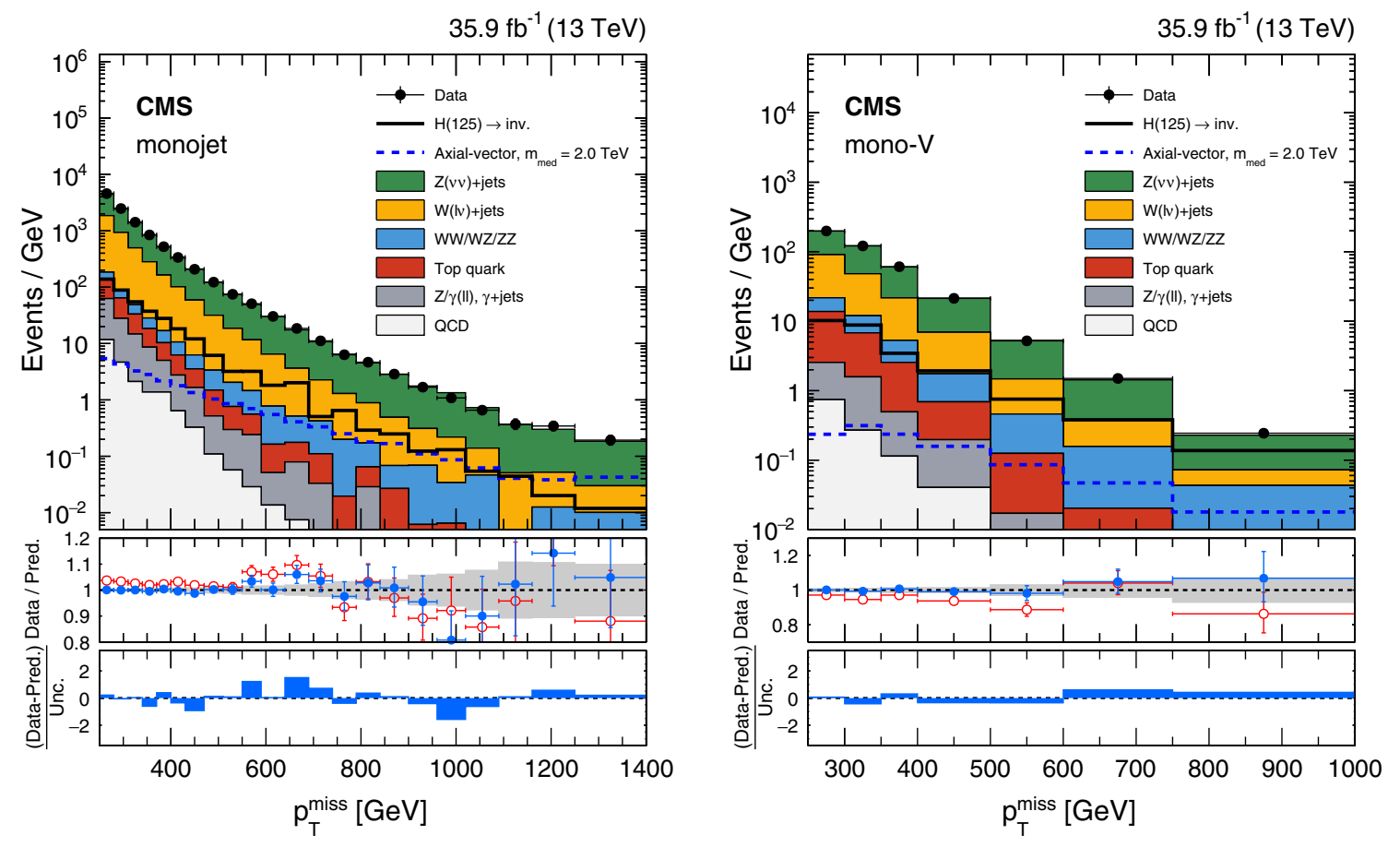

FIG. 9. Observed $p_{\mathrm{T}}^{\text {miss }}$ distribution in the monojet (left) and mono- $V$ (right) signal regions compared with the postfit background expectations for various SM processes. The last bin includes all events with $p_{\mathrm{T}}^{\text {miss }}>1250(750) \mathrm{GeV}$ for the monojet (mono- $V$ ) category. The expected background distributions are evaluated after performing a combined fit to the data in all the control samples, as well as in the signal region. The fit is performed assuming the absence of any signal. Expected signal distributions for the $125 \mathrm{GeV}$ Higgs boson decaying exclusively to invisible particles and a $2 \mathrm{TeV}$ axial-vector mediator decaying to $1 \mathrm{GeV} \mathrm{DM}$ particles are overlaid. The description of the lower panels is the same as in Fig. 5. 

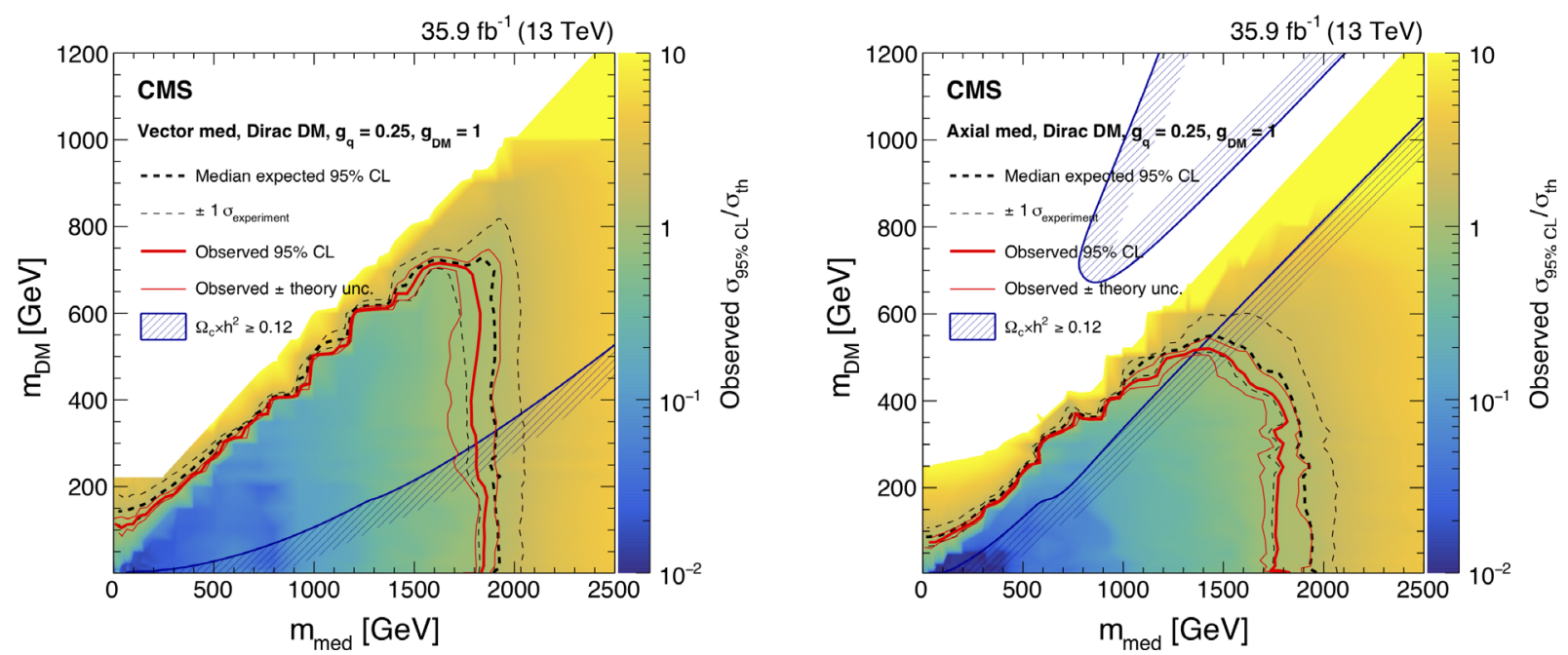

FIG. 10. Exclusion limits at $95 \% \mathrm{CL}$ on $\mu=\sigma / \sigma_{\text {th }}$ in the $m_{\mathrm{med}}-m_{\mathrm{DM}}$ plane assuming vector (left) and axial-vector (right) mediators. The solid (dotted) red (black) line shows the contour for the observed (expected) exclusion. The solid contours around the observed limit and the dashed contours around the expected limit represent one standard deviation due to theoretical uncertainties in the signal cross section and the combination of the statistical and experimental systematic uncertainties, respectively. Constraints from the Planck satellite experiment [97] are shown as dark blue contours; in the shaded area, DM is overabundant.

signal regions, respectively. The correlations between the predicted background yields across all the $p_{\mathrm{T}}^{\text {miss }}$ bins in the two signal regions are shown in Figs. 20 and 21. The expected yields together with the correlations can be used with the simplified likelihood approach detailed in Ref. [92] to reinterpret the results for models not studied in this paper.

Figure 9 shows a comparison between data and the postfit background predictions in the signal region in the monojet and mono- $V$ categories, where the fit is performed under the background-only hypothesis including signal region events in the likelihood. The limits on the production cross section of the various models described below are set after comparing this fit with an alternative one assuming the presence of signal.

\section{A. Dark matter interpretation}

The results are interpreted in terms of simplified $s$ channel DM models assuming a vector, axial-vector, scalar, or pseudoscalar mediator decaying into a pair of fermionic DM particles. The coupling of the mediators to the DM is
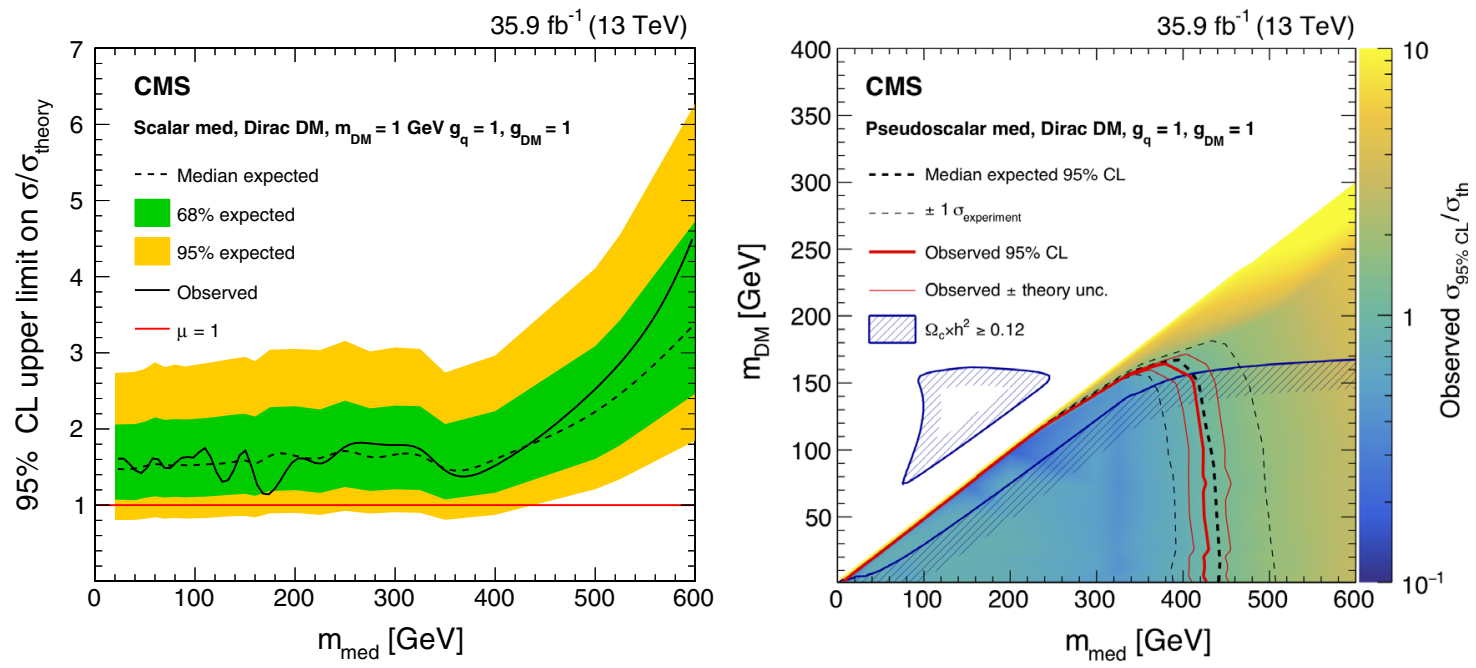

FIG. 11. Expected (dotted black line) and observed (solid black line) 95\% CL upper limits on the signal strength $\mu=\sigma / \sigma_{\text {th }}$ as a function of the mediator mass for the scalar mediators (left) for $m_{\mathrm{DM}}=1 \mathrm{GeV}$. The horizontal red line denotes $\mu=1$. Exclusion limits at $95 \% \mathrm{CL}$ on $\mu=\sigma / \sigma_{\mathrm{th}}$ in the $m_{\mathrm{med}}-m_{\mathrm{DM}}$ plane assuming pseudoscalar mediators (right). The solid (dashed) red (back) line shows the contours for the observed (expected) exclusion. Constraints from the Planck satellite experiment [97] are shown with the dark blue contours; in the shaded area, DM is overabundant. 

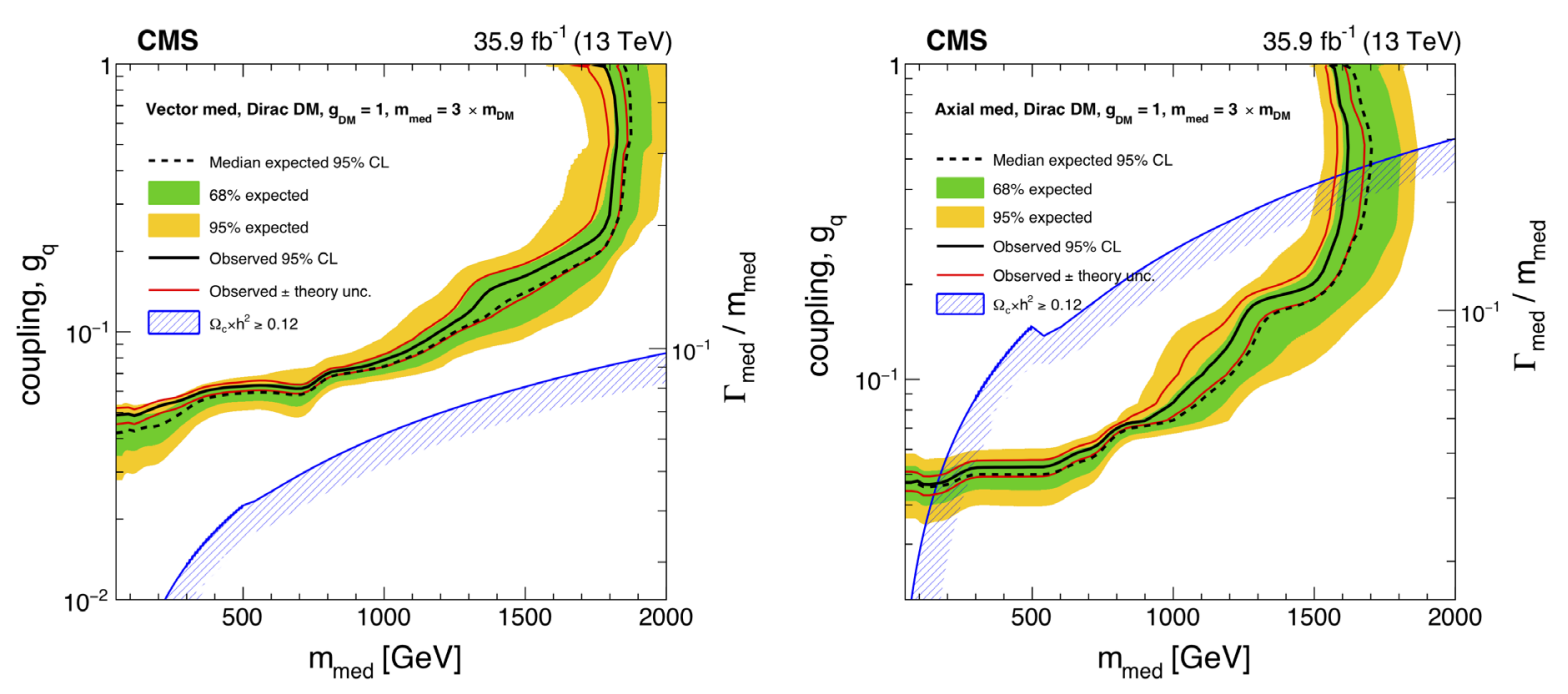

FIG. 12. Exclusion limits at $95 \% \mathrm{CL}$ on $\mu=\sigma / \sigma_{\mathrm{th}}$ in the $m_{\mathrm{med}}-g_{\mathrm{q}}$ plane assuming vector (left) and axial-vector (right) mediators. The widths shown on the axis correspond to mediator masses above $400 \mathrm{GeV}$, where the top quark decay channel is fully open. For the mediator masses below the top quark decay-channel threshold, the width is $9 \%$ less. The solid (dotted) black line shows the contour for the observed (expected) exclusion. The solid red contours around the observed limit represent one standard deviation due to theoretical uncertainties in the signal cross section. Constraints from the Planck satellite experiment [97] are shown as dark blue contours; in the shaded area, DM is overabundant.

assumed to be unity for all four types of mediators. The spin-0 particles are assumed to couple to the quarks with a coupling strength $\left(g_{\mathrm{q}}\right)$ of 1 . In the case of the spin-1 mediators, $g_{\mathrm{q}}$ is taken to be 0.25 . The choice of all the signal model parameters follows the recommendations from Ref. [93]. Uncertainties of $20 \%$ and $30 \%$ are assigned to the inclusive signal cross section in the case of the spin-1 and spin- 0 mediators, respectively. These estimates include the renormalization and factorization scale uncertainties, as well as the PDF uncertainty.

Upper limits are computed at $95 \% \mathrm{CL}$ on the ratio of the measured signal cross section to the predicted one, denoted by $\mu=\sigma / \sigma_{\mathrm{th}}$, with the $\mathrm{CL}_{\mathrm{s}}$ method $[94,95]$, using the asymptotic approximation [96]. Limits are obtained as a function of the mediator mass $\left(m_{\mathrm{med}}\right)$ and the DM mass $\left(m_{\mathrm{DM}}\right)$. Figure 10 shows the exclusion contours in the
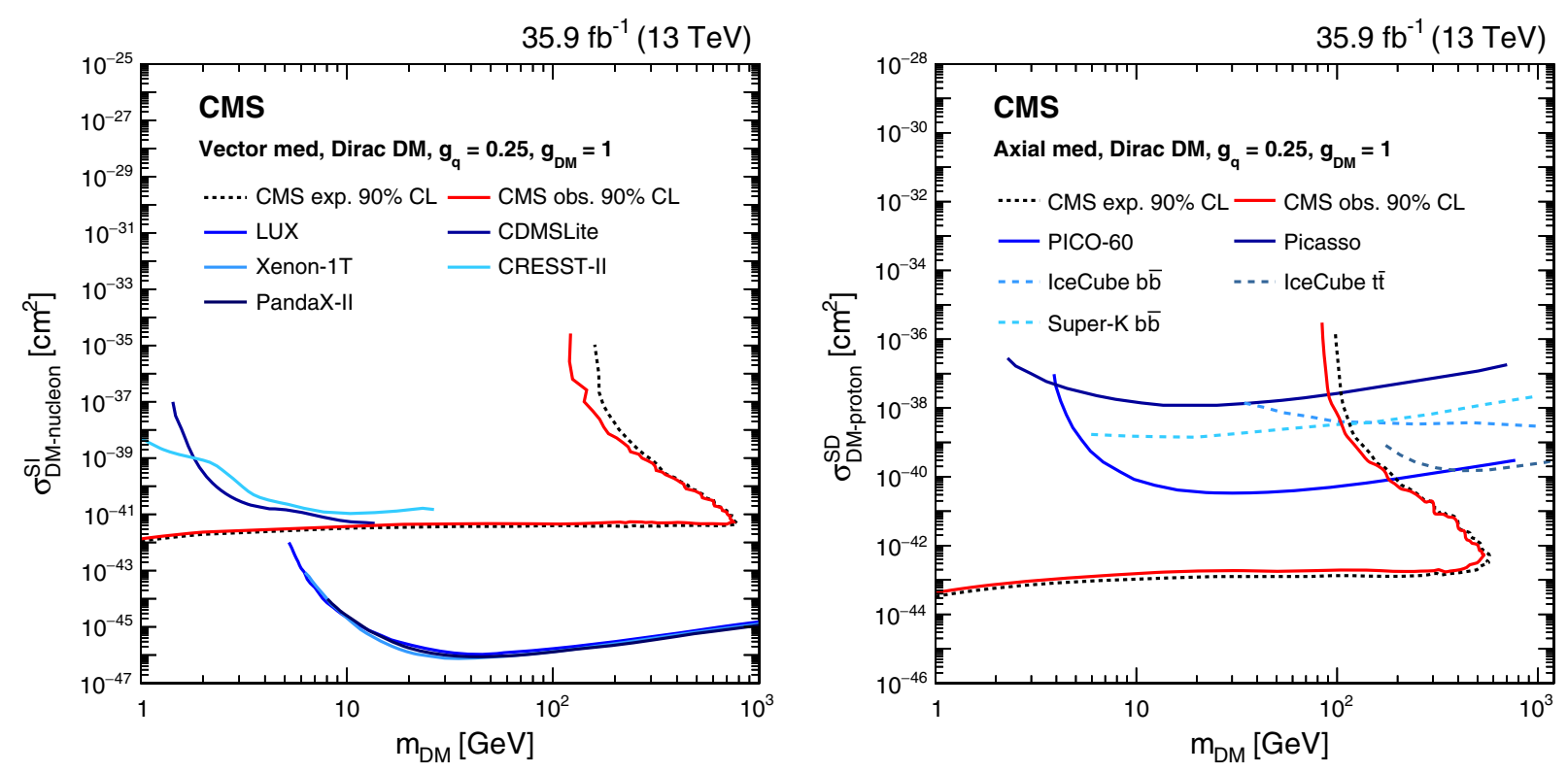

FIG. 13. Exclusion limits at $90 \% \mathrm{CL}$ in the $m_{\mathrm{DM}} \mathrm{vs} \sigma_{\mathrm{SI} / \mathrm{SD}}$ plane for vector (left) and axial-vector (right) mediator models. The solid red (dotted black) line shows the contour for the observed (expected) exclusion in this search. Limits from CDMSLite [102], LUX [103], XENON-1T [104], PANDAX-II [105], and CRESST-II [106] are shown for the vector mediator. Limits from Picasso [107], PICO-60 [108], IceCube [109], and Super-Kamiokande [110] are shown for the axial-vector mediator. 
$m_{\text {med }}-m_{\text {DM }}$ plane for the vector and axial-vector mediators. Mediator masses up to $1.8 \mathrm{TeV}$ and $\mathrm{DM}$ masses up to 700 and $500 \mathrm{GeV}$ are excluded for the vector and axial-vector models, respectively. Figure 11 shows the limits for the scalar mediators as a function of the mediator mass, for a fixed $\mathrm{DM}$ mass of $1 \mathrm{GeV}$ and the exclusion contours in the $m_{\text {med }}-m_{\text {DM }}$ plane for pseudoscalar mediators, respectively. Pseudoscalar mediator (dark matter) masses up to 400 (150) $\mathrm{GeV}$ are excluded at 95\% CL. A direct comparison of the results for simplified DM models of this paper to the one presented in Ref. [14] can be seen in Figs. 22 and 23.

The results for vector, axial-vector, and pseudoscalar mediators are compared to constraints from the observed cosmological relic density of DM as determined from measurements of the cosmic microwave background by the Planck satellite experiment [97]. The expected DM abundance is estimated, separately for each model, using the thermal freeze-out mechanism implemented in the MADDM [98] framework and compared to the observed cold DM density $\Omega_{c} h^{2}=0.12$ [99], where $\Omega_{c}$ is the DM relic abundance and $h$ is the Hubble constant.

In addition to scanning the $m_{\text {med }}-m_{\text {DM }}$ plane, for a fixed $g_{\mathrm{q}}$ value, the analysis interprets the results in the $m_{\text {med }}-g_{\mathrm{q}}$ plane for a fixed ratio of $m_{\text {med }} / m_{\mathrm{DM}}=3$. The ratio is chosen to ensure a valid relic abundance solution for every allowed $g_{\mathrm{q}}$ value scanned for a spin-1 simplified model. Quark couplings down to 0.05 for mediator masses at $50 \mathrm{GeV}$ are excluded for the spin-1 simplified models as shown in Fig. 12.

The exclusion contours obtained from the simplified DM models are translated to $90 \%$ CL upper limits on the spin-independent/spin-dependent $\left(\sigma_{\mathrm{SI} / \mathrm{SD}}\right)$ DM-nucleon scattering cross sections using the approach outlined in Refs. [19,36,100]. The results for the vector and axialvector mediators are compared with the results of direct searches in Fig. 13. This search provides the most stringent constraints for vector mediators, for DM particle masses below $5 \mathrm{GeV}$. For axial-vector mediators, the sensitivity achieved in this search provides stronger constraints up to a DM particle mass of $550 \mathrm{GeV}$ than those obtained from direct searches. For pseudoscalar mediators, the 90\% CL upper limits as shown in Fig. 14 are translated to velocityaveraged DM annihilation cross section $(\langle\sigma v\rangle)$ and are compared to the indirect detection results from the FermiLAT Collaboration [101]. The collider results provide stronger constraints for DM masses less than $150 \mathrm{GeV}$.

\section{Fermion portal dark matter interpretation}

The total production cross section in the fermion portal DM model has an exponential (linear) dependence on the mass of the new scalar mediator $m_{\phi_{\mathrm{u}}}$ (mass of the DM candidate $m_{\chi}$ ). The middle diagram shown in Fig. 1 represents the main production mechanism for small $m_{\phi_{\mathrm{u}}}$ values, whereas the right diagram contributes to the total

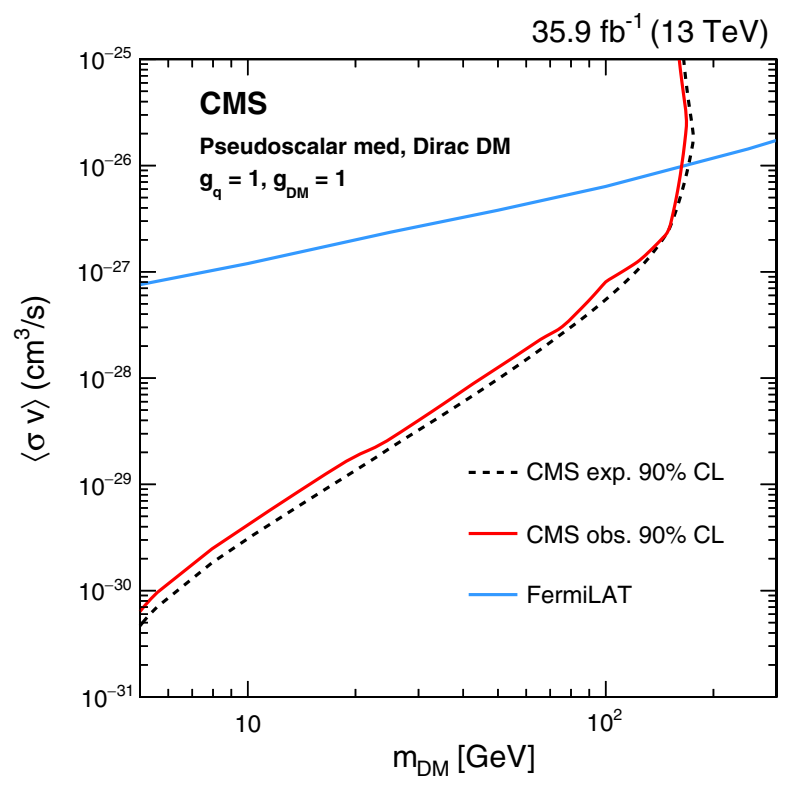

FIG. 14. For the pseudoscalar mediator, limits are compared to the velocity-averaged DM annihilation cross section upper limits from Fermi-LAT [101]. There are no comparable limits from direct detection experiments, as the scattering cross section between DM particles and SM quarks is suppressed at nonrelativistic velocities for a pseudoscalar mediator [111,112].

cross section for $m_{\phi_{\mathrm{u}}}>1 \mathrm{TeV}$. The region where $m_{\phi_{\mathrm{u}}}<$ $m_{\chi}$ is not considered in the search, because of the reduced production cross section of the model. The upper limits on the signal strength are set as a function of $m_{\phi_{\mathrm{u}}}$ and $m_{\chi}$.

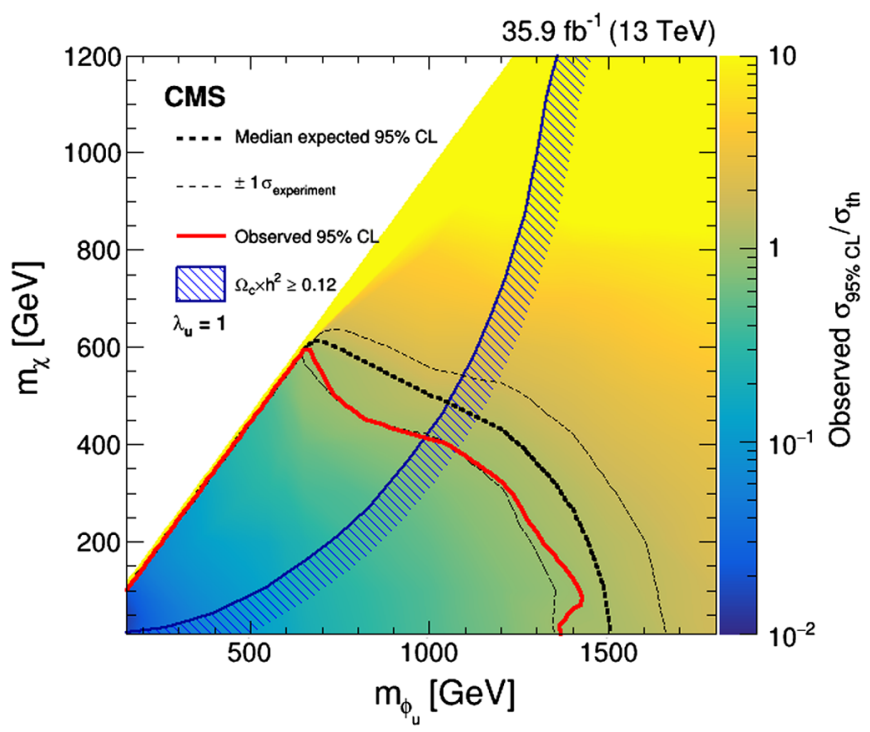

FIG. 15. The 95\% CL expected (black dashed line) and observed (red solid line) upper limits on $\mu=\sigma / \sigma_{\text {th }}$ in the context of the fermion portal DM model, for Dirac DM particles with coupling strengths to the up quark corresponding to $\lambda_{\mathrm{u}}=1$ in the $m_{\phi_{\mathrm{u}}}-m_{\chi}$ plane. Constraints from the Planck satellite experiment [97] are shown as dark blue contours; in the shaded area, DM is overabundant. 

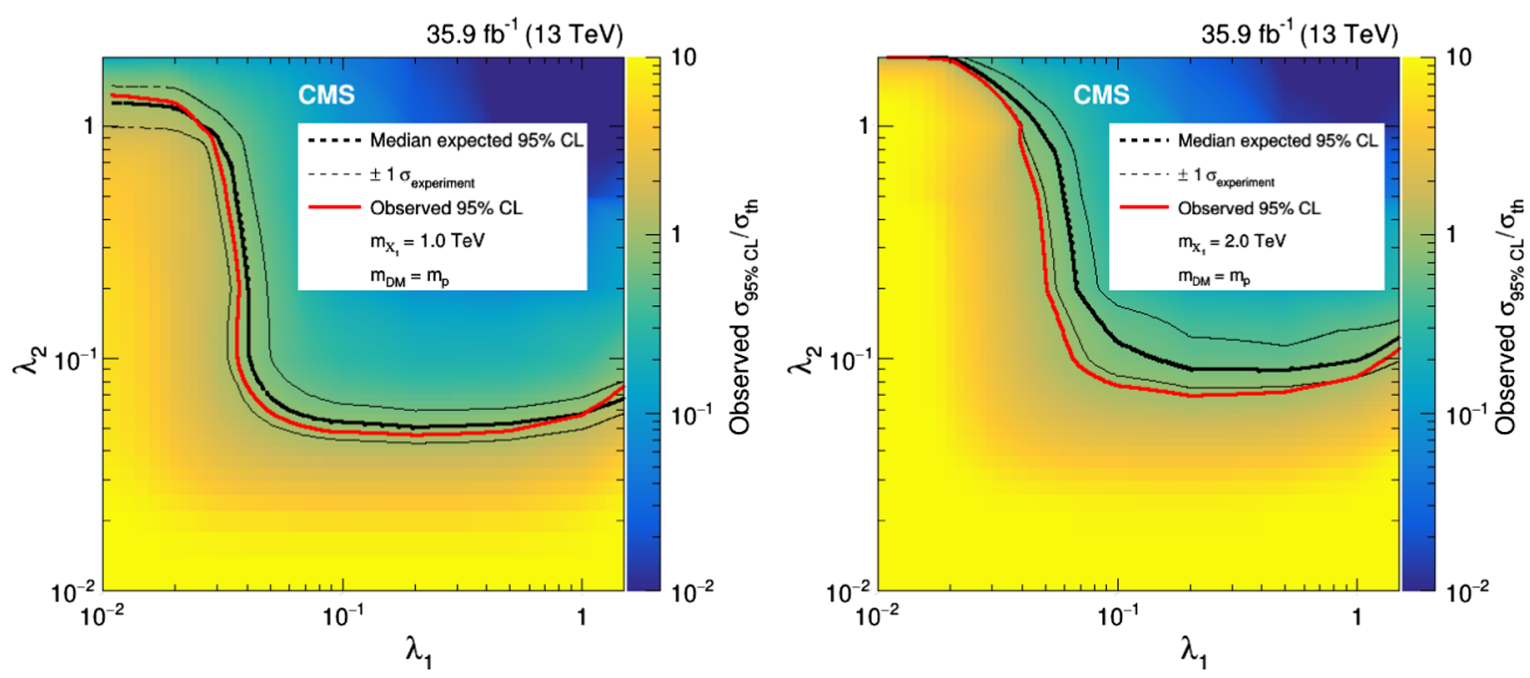

FIG. 16. Expected (black line) and observed (red line) 95\% CL upper limits on the signal strength $\mu=\sigma / \sigma_{\text {th }}$, in the context of a nonthermal dark matter model. Results are reported in the $\lambda_{1}-\lambda_{2}$ plane, which represents the coupling strength of the interaction of the new scalar mediator with down-type quarks and DM with up-type quarks, respectively. Limits are shown for $m_{\mathrm{X}_{1}}$ of 1 (left) and $2 \mathrm{TeV}$ (right).

Figure 15 shows the exclusion contours in the $m_{\phi_{\mathrm{u}}}-m_{\chi}$ plane, for which the coupling strength $\lambda_{\mathrm{u}}$ of the interaction between the scalar mediator and up-type quarks is fixed at unity. The results are also compared to constraints from the observed cosmological relic density of DM, obtained by the Planck satellite experiment, for the allowed values of $m_{\phi_{\mathrm{u}}}$ and $m_{\chi}$ [20]. In this search, mediator (dark matter) masses up to $1.4(0.6) \mathrm{TeV}$ are excluded.

\section{Nonthermal dark matter interpretation}

This search is also interpreted in the context of the nonthermal DM model where the DM candidate is not parity protected and therefore could be singly produced. Such production leads to signatures with an energetic jet and large $p_{\mathrm{T}}^{\text {miss }}$ of which the distribution is characterized by a Jacobian-like shape, which exhibits a peak at half of the mediator mass. Therefore, multiple mediator mass points have been studied. The search is restricted to a coupling range of 0.01-1.5 for $\lambda_{1}$ and 0.01-2.0 for $\lambda_{2}$ to ensure the mediator width is less than about $30 \%$ of its mass. Within these bounds, no significant excesses were found, and limits are reported as a function of coupling strength parameters $\lambda_{1}$ and $\lambda_{2}$ for two reference mediator masses $m_{\mathrm{X}_{1}}$ of 1 and $2 \mathrm{TeV}$. Figure 16 shows the exclusion contours in the $\lambda_{1}-\lambda_{2}$ plane.

\section{B. Invisible decays of the Higgs boson interpretation}

The results of this search are further interpreted in terms of an upper limit on the production cross section and branching fraction, $\mathcal{B}(\mathrm{H} \rightarrow$ inv $)$, where the Higgs boson is produced through gluon fusion $(\mathrm{ggH})$ along with a jet, in association with a vector boson $(\mathrm{ZH}, \mathrm{WH})$, or through vector boson fusion (VBF). The predictions for the Higgs boson production cross section and the corresponding theoretical uncertainties are taken from the recommendations of the LHC Higgs cross section working group [113]. The observed (expected) 95\% CL upper limit on the invisible branching fraction of the Higgs boson, $\sigma \times \mathcal{B}(\mathrm{H} \rightarrow \mathrm{inv}) / \sigma_{\mathrm{SM}}$, is found to be $53 \%(40 \%)$. The

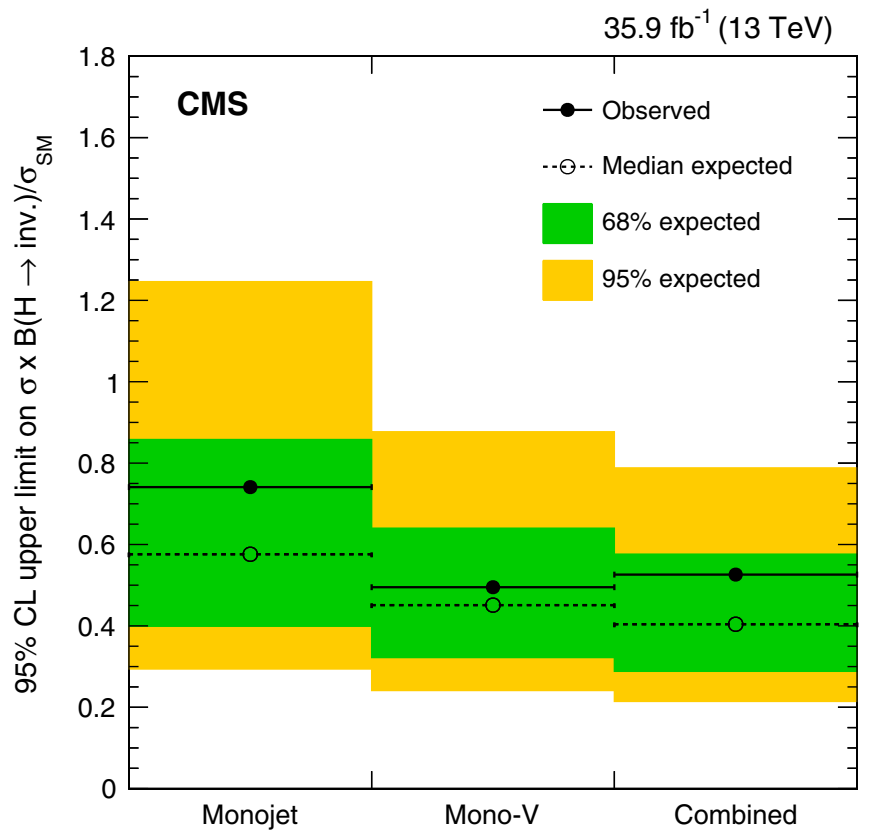

FIG. 17. Expected (dotted line) and observed (solid line) 95\% CL upper limits on the invisible branching fraction of the $125 \mathrm{GeV}$ SM-like Higgs boson. Limits are shown for the monojet and mono- $V$ categories separately and also for their combination. 
TABLE VI. Expected and observed 95\% CL upper limits on the invisible branching fraction of the Higgs boson. Limits are tabulated for the monojet and mono- $V$ categories separately and for their combination. The one standard deviation uncertainty range in the expected limits is listed. The expected composition of the production modes of a Higgs boson with a mass of $125 \mathrm{GeV}$ is summarized, assuming SM production cross sections.

\begin{tabular}{lccc}
\hline \hline Category & Observed (expected) & $68 \%$ expected & Expected signal composition \\
\hline Monojet & $0.74(0.57)$ & $0.40-0.86$ & $72.8 \%$ ggH, 21.5\% VBF, \\
Mono- $V$ & $0.49(0.45)$ & & $3.3 \% \mathrm{WH}, 1.9 \% \mathrm{ZH}, 0.6 \% \mathrm{ggZH}$ \\
Combined & $0.53(0.40)$ & $0.32-0.64$ & $38.7 \% \mathrm{ggH}, 7.0 \% \mathrm{VBF}$, \\
\hline \hline
\end{tabular}

limits are summarized in Fig. 17, while Table VI shows the individual limits for the monojet and mono- $V$ categories.

\section{ADD model interpretation}

The 95\% CL lower limits on the fundamental Planck scale $M_{\mathrm{D}}$ of the ADD model are presented as a function of the number of extra spatial dimensions $n$. The efficiency of the full event selection in the monojet (mono- $V$ ) category for this model ranges between 15\% (1\%) and 20\% (1.5\%) depending on the values of the parameters $M_{\mathrm{D}}$ and $n$. An upper limit on the signal strength $\mu=\sigma / \sigma_{\text {th }}$ is presented for the ADD graviton production for $n=2 \mathrm{EDs}$, as a function of $M_{\mathrm{D}}$ in Fig. 18. In addition, Fig. 18 shows the observed exclusion on $M_{\mathrm{D}}$ which varies from $9.9 \mathrm{TeV}$ for $n=2$ to $5.3 \mathrm{TeV}$ for $n=6$. The results of this search are also compared to earlier ones obtained by the CMS Collaboration with Run 1 data corresponding to an integrated luminosity of $19.7 \mathrm{fb}^{-1}$ at a center-of-mass energy of $8 \mathrm{TeV}$ [10]. The upper limits on the signal production

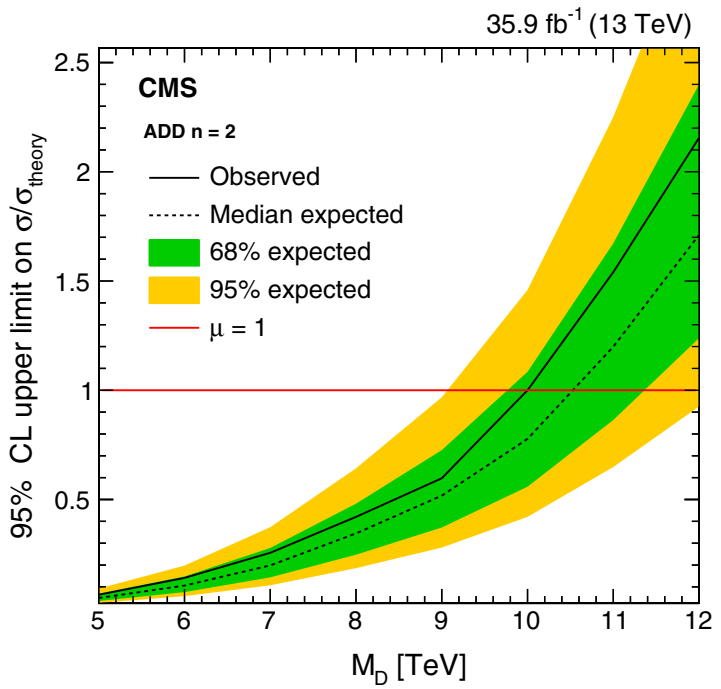

cross section and $M_{\mathrm{D}}$ exclusions are also provided in Table VII as a function of the number of extra dimensions. Compared to previous CMS publications in this channel, the lower limits on $M_{\mathrm{D}}$ show a factor of 2 improvement.

\section{SUMMARY}

A search for DM particles, invisible decays of a SM-like Higgs boson, and extra spatial dimensions is presented using events with one or more energetic jets and large missing transverse momentum in proton-proton collisions recorded at $\sqrt{s}=13 \mathrm{TeV}$, using a sample of data corresponding to an integrated luminosity of $35.9 \mathrm{fb}^{-1}$. Events are categorized based on whether jets are produced directly in hard scattering as initial-state radiation or originate from merged quarks from a decay of a highly Lorentz-boosted $W$ or $Z$ boson. No excess of events is observed compared to the SM background expectations in either of these two categories.

Limits are computed on the DM production cross section using simplified models in which DM production is

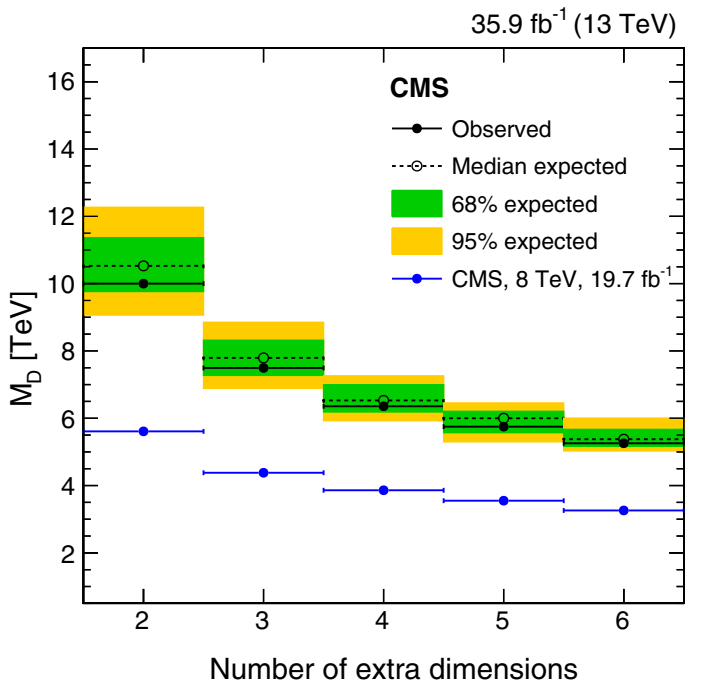

FIG. 18. The 95\% CL expected (dotted) and observed (solid) upper limits on the signal strength $\mu=\sigma / \sigma_{\text {th }}$ for ADD graviton production (left), as a function of fundamental Planck scale $\left(M_{\mathrm{D}}\right)$ for $n=2$, where $n$ is the number of extra spatial dimensions. The 95\% CL expected (dotted) and observed (solid) lower limits (right) on $M_{\mathrm{D}}$ as a function of $n$ in the ADD model. The results are also compared to earlier ones obtained by the CMS Collaboration with data corresponding to an integrated luminosity of $19.7 \mathrm{fb}^{-1}$ at a center-of-mass energy of $8 \mathrm{TeV}$ [10] (blue points). 
TABLE VII. Upper limits on the signal production cross section in the ADD model and lower limits on $M_{\mathrm{D}}$, both as functions of the number of extra spatial dimensions $(n)$.

\begin{tabular}{ccc}
\hline \hline & $\begin{array}{c}\text { Observed (expected) } \\
\text { cross section exclusion (pb) }\end{array}$ & $\begin{array}{c}\text { Observed (expected) } \\
M_{\mathrm{D}} \text { exclusions (TeV) }\end{array}$ \\
\hline 2 & $0.28(0.22)$ & $9.9(10.5)$ \\
3 & $0.18(0.15)$ & $7.5(7.8)$ \\
4 & $0.15(0.13)$ & $6.3(6.5)$ \\
5 & $0.13(0.11)$ & $5.7(6.0)$ \\
6 & $0.13(0.10)$ & $5.3(5.4)$ \\
\hline \hline
\end{tabular}

mediated by spin- 1 and spin- 0 particles. Vector and axialvector (pseudoscalar) mediators with masses up to 1.8 (0.4) $\mathrm{TeV}$ are excluded at 95\% C.L. Similarly, limits are also presented for the parameters of the fermion portal DM model, and an exclusion up to $1.4 \mathrm{TeV}$ on the mediator mass is observed at $95 \%$ confidence level. The first limits on the DM production at a particle collider in the nonthermal DM model are obtained and presented in the coupling strength plane. Furthermore, an observed (expected) 95\% confidence level upper limit of 0.53 $(0.40)$ is set for the invisible branching fraction of an SM-like $125 \mathrm{GeV}$ Higgs boson, assuming the SM production cross section. Lower limits are also computed on the fundamental Planck scale $M_{\mathrm{D}}$ in the context of the Arkani-Hamed, Dimopoulos, and Dvali model with large extra spatial dimensions, which varies from $9.9 \mathrm{TeV}$ for $n=2$ to $5.3 \mathrm{TeV}$ for $n=6$ at $95 \%$ C.L., where $n$ is the number of extra spatial dimensions. These limits provide the most stringent direct constraints on the fundamental Planck scale to date.

\section{ACKNOWLEDGMENTS}

We congratulate our colleagues in the CERN accelerator departments for the excellent performance of the LHC and thank the technical and administrative staffs at CERN and at other CMS institutes for their contributions to the success of the CMS effort. In addition, we gratefully acknowledge the computing centers and personnel of the Worldwide LHC Computing Grid for delivering so effectively the computing infrastructure essential to our analyses. Finally, we acknowledge the enduring support for the construction and operation of the LHC and the CMS detector provided by the following funding agencies: BMWFW and FWF (Austria); FNRS and FWO (Belgium); CNPq, CAPES, FAPERJ, and FAPESP (Brazil); MES (Bulgaria); CERN; CAS, MoST, and NSFC (China); COLCIENCIAS (Colombia); MSES and CSF (Croatia); RPF (Cyprus); SENESCYT (Ecuador); MoER, ERC IUT, and ERDF (Estonia); Academy of Finland, MEC, and HIP (Finland); CEA and CNRS/IN2P3 (France); BMBF, DFG, and HGF (Germany); GSRT (Greece); OTKA and NIH (Hungary); DAE and DST (India); IPM (Iran); SFI
(Ireland); INFN (Italy); MSIP and NRF (Republic of Korea); LAS (Lithuania); MOE and UM (Malaysia); BUAP, CINVESTAV, CONACYT, LNS, SEP, and UASLP-FAI (Mexico); MBIE (New Zealand); PAEC (Pakistan); MSHE and NSC (Poland); FCT (Portugal); JINR (Dubna); MON, RosAtom, RAS, RFBR and RAEP (Russia); MESTD (Serbia); SEIDI, CPAN, PCTI and FEDER (Spain); Swiss Funding Agencies (Switzerland); MST (Taipei); ThEPCenter, IPST, STAR, and NSTDA (Thailand); TUBITAK and TAEK (Turkey); NASU and SFFR (Ukraine); STFC (United Kingdom); and DOE and NSF (USA). Individuals have received support from the Marie-Curie programme and the European Research Council and Horizon 2020 Grant, Grant No. 675440 (European Union); the Leventis Foundation; the A.P. Sloan Foundation; the Alexander von Humboldt Foundation; the Belgian Federal Science Policy Office; the Fonds pour la Formation à la Recherche dans l'Industrie et dans l'Agriculture (FRIA-Belgium); the Agentschap voor Innovatie door Wetenschap en Technologie (IWTBelgium); the Ministry of Education, Youth and Sports (MEYS) of the Czech Republic; the Council of Science and Industrial Research, India; the HOMING PLUS program of the Foundation for Polish Science, cofinanced from European Union, Regional Development Fund, the Mobility Plus program of the Ministry of Science and Higher Education, the National Science Center (Poland), Contracts No. Harmonia 2014/14/M/ST2/00428, No. Opus 2014/13/B/ST2/02543, No. 2014/15/B/ST2/03998, No. 2015/19/B/ST2/02861, and No. Sonata-bis 2012/07/ E/ST2/01406; the National Priorities Research Program by Qatar National Research Fund; the Programa ClarínCOFUND del Principado de Asturias; the Thalis and Aristeia programmes cofinanced by EU-ESF and the Greek NSRF; the Rachadapisek Sompot Fund for Postdoctoral Fellowship, Chulalongkorn University and the Chulalongkorn Academic into Its 2nd Century Project Advancement Project (Thailand); and the Welch Foundation, Contract No. C-1845.

\section{APPENDIX: ADDITIONAL MATERIAL}

Another important cross-check of the application of $p_{\mathrm{T}^{-}}$ dependent NLO QCD and EW corrections is represented by the agreement between data and simulation in the ratio of $Z+$ jets events to both $\gamma+$ jets events and $W+$ jets events in the control samples as a function of boson $p_{\mathrm{T}}$.

Figure 19 shows the ratio between $Z(\mu \mu)+$ jets and $\gamma+$ jets, and the ratio of $Z(\mu \mu)+$ jets and $W(\mu \nu)+$ jets events as a function of the boson $p_{\mathrm{T}}$, for the monojet category. While we do not explicitly use a $W(\mu \nu)+$ jets $/ \gamma+$ jets constraint in the analysis, the two cross sections are connected through the $Z+$ jets $/ \gamma+$ jets and $Z+$ jets $/ W+$ jets constraints. Therefore, it is instructive to examine the data-to-simulation comparison for the $W(\mu \nu)+$ jets $/ \gamma+$ jets ratio. This is shown in the same 
figure. Good agreement is observed between data and simulation after the application of NLO corrections.

The correlations between the predicted background yields across all the $p_{\mathrm{T}}^{\text {miss }}$ bins in the two signal regions are shown in Figs. 20 and 21. These results can be used with the simplified likelihood approach detailed in Ref. [92] for reinterpretations in terms of models not studied in this paper.
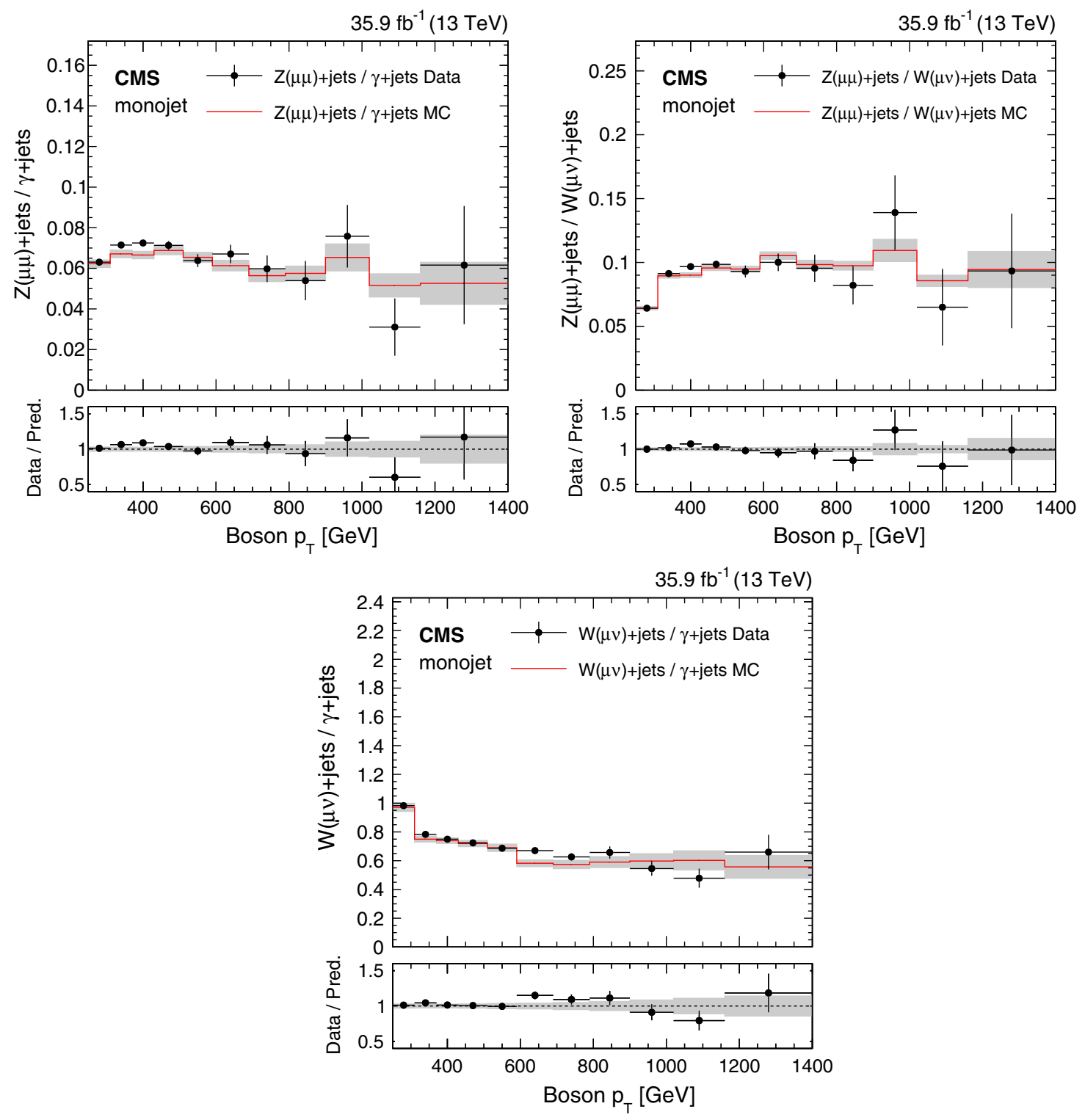

FIG. 19. Comparison between data and Monte Carlo simulation of the $Z(\mu \mu) / \gamma+$ jets, $Z(\mu \mu) / W(\mu \nu)$, and $W(\mu \nu) / \gamma+$ jets ratios, as a function of boson $p_{\mathrm{T}}$, in the monojet category. In the ratio panel, ratios of data with the prefit background prediction are shown. The gray bands include both the prefit systematic uncertainties and the statistical uncertainty in the simulation. 


\section{CMS \\ $35.9 \mathrm{fb}^{-1}(13 \mathrm{TeV})$}

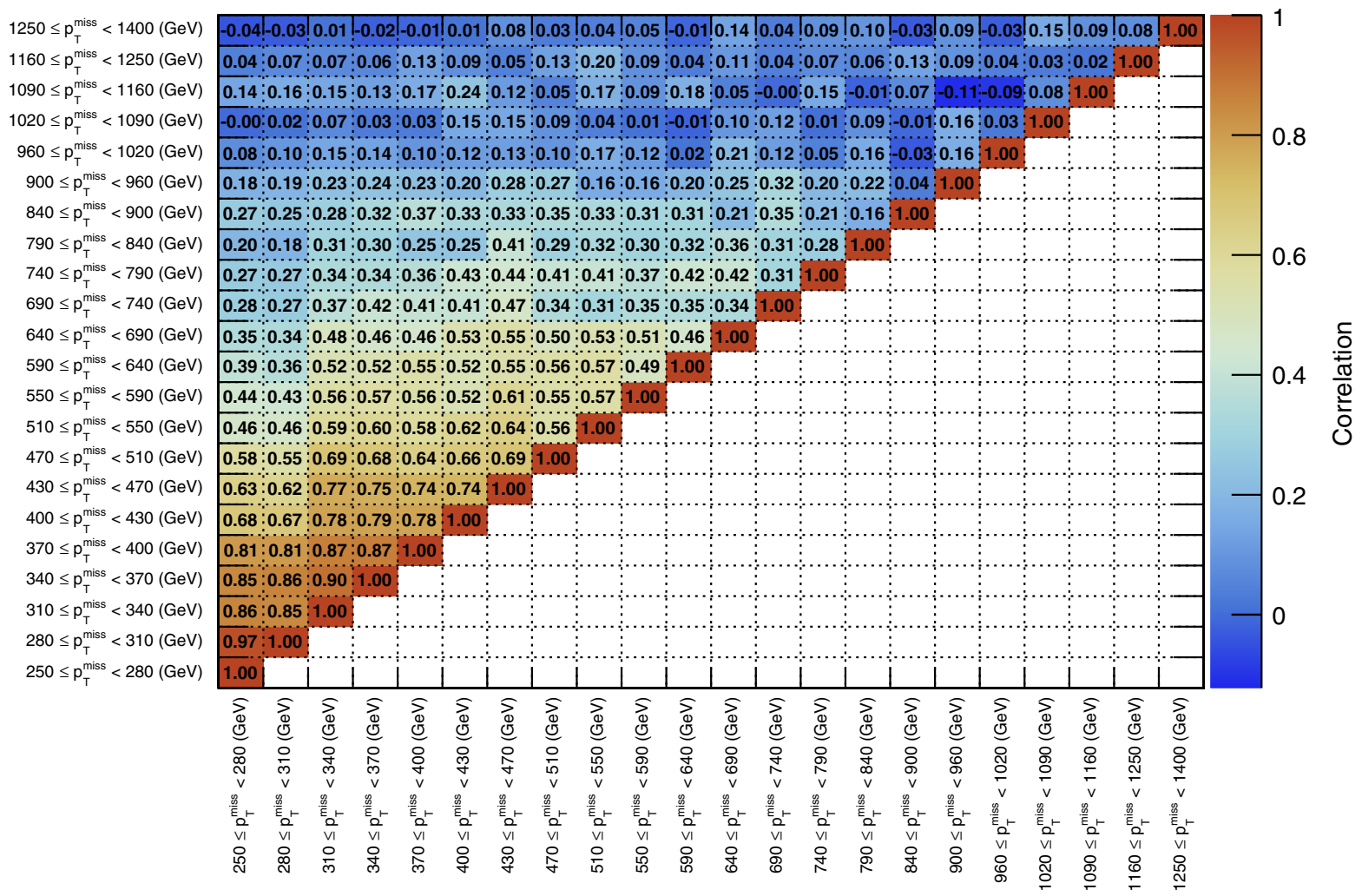

FIG. 20. Correlations between the predicted background yields in all the $E_{\mathrm{T}}^{\mathrm{miss}}$ bins of the monojet signal region. The boundaries of the $E_{\mathrm{T}}^{\text {miss }}$ bins, expressed in $\mathrm{GeV}$, are shown at the bottom and on the left.

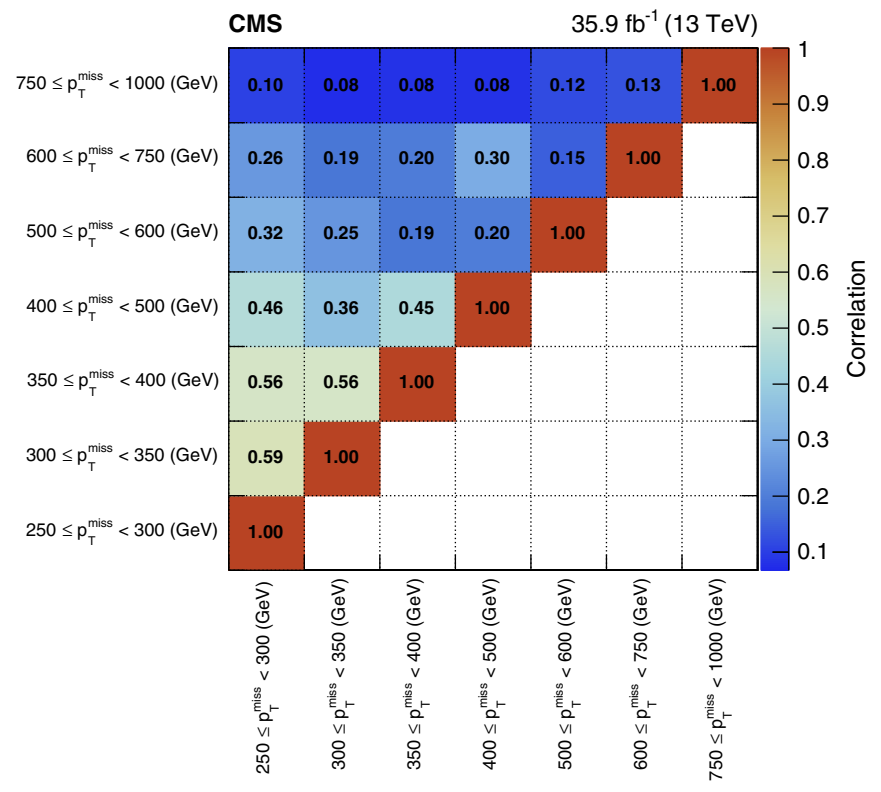

FIG. 21. Correlations between the predicted background yields in all the $E_{\mathrm{T}}^{\mathrm{miss}}$ bins of the mono- $V$ signal region. The boundaries of the $E_{\mathrm{T}}^{\text {miss }}$ bins, expressed in $\mathrm{GeV}$, are shown at the bottom and on the left. 

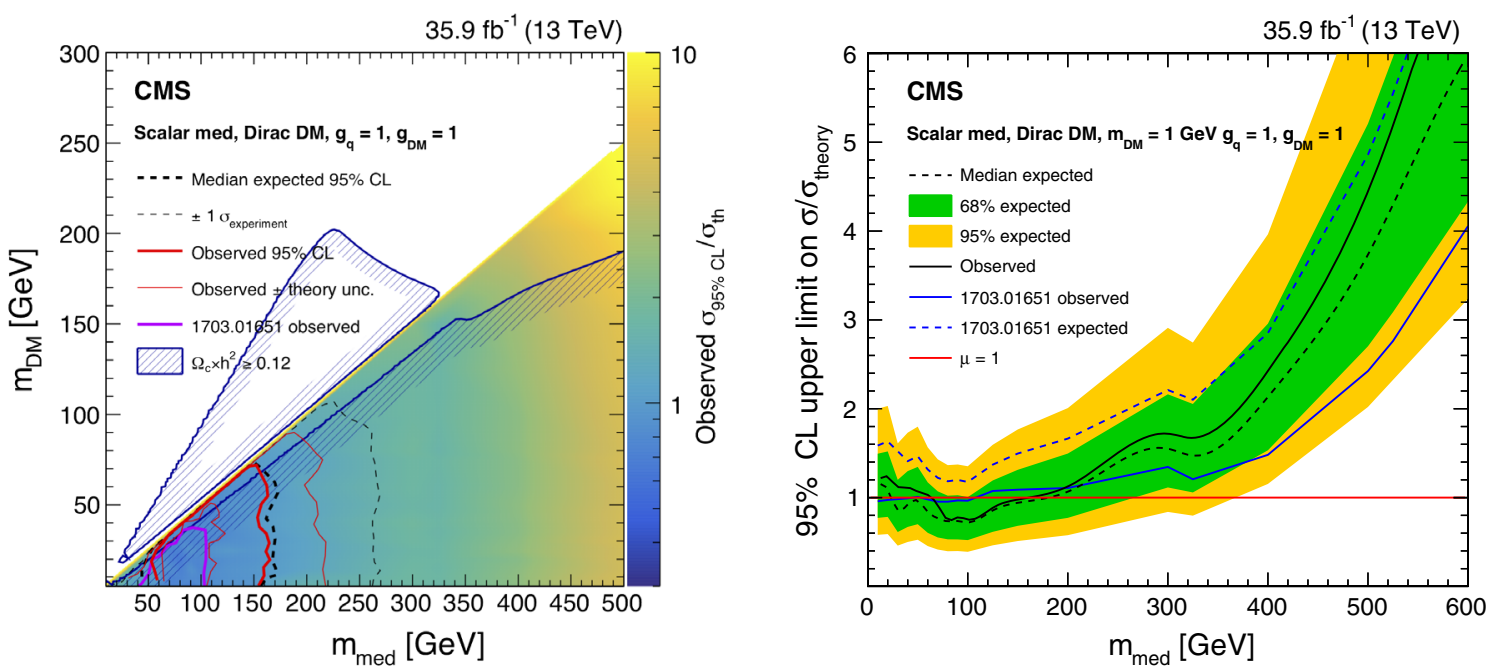

FIG. 22. Exclusion limits at $95 \% \mathrm{CL}$ on $\mu=\sigma / \sigma_{\mathrm{th}}$ in the $m_{\mathrm{med}}-m_{\mathrm{DM}}$ plane assuming scalar mediators (left) allowing for vector boson couplings simulated at LO in QCD. The solid (dotted) red (black) line shows the contour for the observed (expected) exclusion. The solid contours around the observed limit and the dashed contours around the expected limit represent one standard deviation due to theoretical uncertainties in the signal cross section and the quadratic sum of the statistical and experimental systematic uncertainties, respectively. Expected and observed sensitivity of the previous CMS publication [14] are also presented. Results of the Planck satellite experiment [97] are shown as dark blue contours. In the shaded area, DM is overabundant. Expected (dotted black line) and observed (solid black line) $95 \% \mathrm{CL}$ upper limits on the signal strength $\mu$ as a function of the mediator mass for the spin-0 models (right).
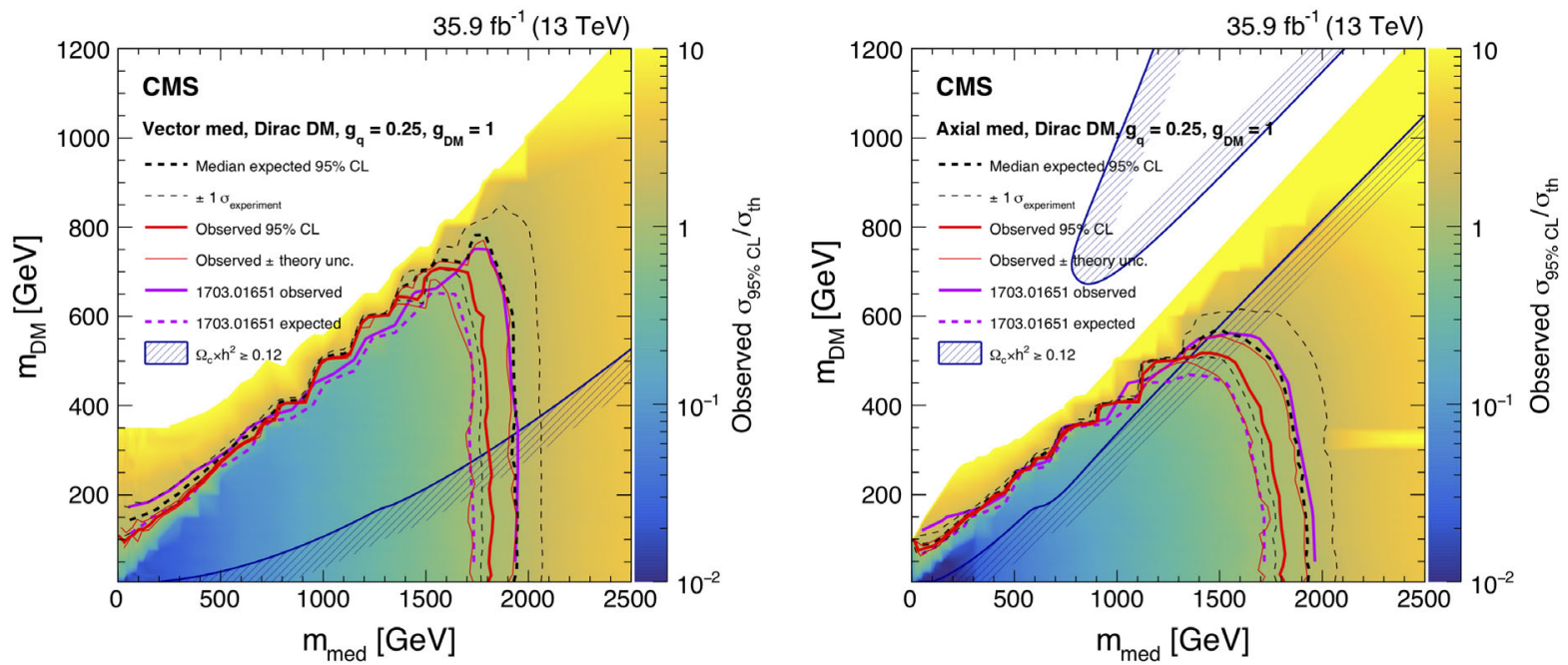

FIG. 23. Exclusion limits at $95 \% \mathrm{CL}$ on $\mu=\sigma / \sigma_{\mathrm{th}}$ in the $m_{\mathrm{med}}-m_{\mathrm{DM}}$ plane assuming vector (left) and axial-vector (right) mediators where the mono- $V$ signal is simulated at LO in QCD. The solid (dotted) red (black) line shows the contour for the observed (expected) exclusion. The solid contours around the observed limit and the dashed contours around the expected limit represent one standard deviation due to theoretical uncertainties in the signal cross section and the quadratic sum of the statistical and experimental systematic uncertainties, respectively. Planck satellite experiment [97] are shown as dark blue contours. In the shaded area DM is overabundant.

TABLE VIII. Monte Carlo generators and perturbative order in QCD used for simulating various signal processes studied in this work and in Ref. [14]

\begin{tabular}{lcc} 
Process & $\begin{array}{c}\text { Monte Carlo generator } \\
\text { (perturbative order in QCD) Ref. [14] }\end{array}$ & $\begin{array}{c}\text { Monte Carlo generator } \\
\text { (perturbative order in QCD) this work }\end{array}$ \\
\hline Monojet (spin-1 mediator) & POWHEG2.0 (NLO) & MADGRAPH5_aMC@NLO 2.2.3 (NLO) \\
Monojet (spin-0 mediator) & POWHEG2.0 (LO) & MADGRAPH5_aMC@ NLO 2.2.3 (NLO) \\
Mono- $V$ (spin-1 mediator) & MADGRAPH5_aMC@NLO 2.2.3 (LO) & MADGRAPH5_aMC@NLO 2.2.3 (NLO) \\
Mono- $V$ (spin-0 mediator) & JHUGENERATOR 5.2.5 & Not used \\
\hline \hline
\end{tabular}


[1] G. Bertone, D. Hooper, and J. Silk, Particle dark matter: Evidence, candidates and constraints, Phys. Rep. 405, 279 (2005).

[2] J. L. Feng, Dark matter candidates from particle physics and methods of detection, Annu. Rev. Astron. Astrophys. 48, 495 (2010).

[3] T. A. Porter, R. P. Johnson, and P. W. Graham, Dark matter searches with astroparticle data, Annu. Rev. Astron. Astrophys. 49, 155 (2011).

[4] N. Arkani-Hamed, S. Dimopoulos, and G. Dvali, The hierarchy problem and new dimensions at a millimeter, Phys. Lett. B 429, 263 (1998).

[5] N. Arkani-Hamed, S. Dimopoulos, and G. R. Dvali, Phenomenology, astrophysics and cosmology of theories with submillimeter dimensions and $\mathrm{TeV}$ scale quantum gravity, Phys. Rev. D 59, 086004 (1999).

[6] I. Antoniadis, K. Benakli, and M. Quiros, Direct collider signatures of large extra dimensions, Phys. Lett. B 460, 176 (1999).

[7] G. Giudice, R. Rattazzi, and J. Wells, Quantum gravity and extra dimensions at high-energy colliders, Nucl. Phys. B544, 3 (1999).

[8] E. A. Mirabelli, M. Perelstein, and M. E. Peskin, Collider Signatures of New Large Space Dimensions, Phys. Rev. Lett. 82, 2236 (1999).

[9] ATLAS Collaboration, Search for Dark Matter in Events with a Hadronically Decaying $W$ or $Z$ Boson and Missing Transverse Momentum in pp Collisions at $\sqrt{s}=8 \mathrm{TeV}$ with the ATLAS Detector, Phys. Rev. Lett. 112, 041802 (2014).

[10] CMS Collaboration, Search for dark matter, extra dimensions, and unparticles in monojet events in protoproton collisions at $\sqrt{s}=8 \mathrm{TeV}$, Eur. Phys. J. C 75, 235 (2015).

[11] ATLAS Collaboration, Search for new phenomena in final states with an energetic jet and large missing transverse momentum in pp collisions at $\sqrt{s}=8 \mathrm{TeV}$ with the ATLAS detector, Eur. Phys. J. C 75, 299 (2015); Erratum, 75, 408(E) (2015).

[12] CMS Collaboration, Search for dark matter in protonproton collisions at $8 \mathrm{TeV}$ with missing transverse momentum and vector boson tagged jets, J. High Energy Phys. 12 (2016) 083.

[13] ATLAS Collaboration, Search for new phenomena in final states with an energetic jet and large missing transverse momentum in $\mathrm{pp}$ collisions at $\sqrt{s}=13 \mathrm{TeV}$ using the ATLAS detector, Phys. Rev. D 94, 032005 (2016).

[14] CMS Collaboration, Search for dark matter produced with an energetic jet or a hadronically decaying $\mathrm{W}$ or $\mathrm{Z}$ boson at $\sqrt{s}=13 \mathrm{TeV}$, J. High Energy Phys. 07 (2017) 014.

[15] ATLAS Collaboration, Search for dark matter and other new phenomena in events with an energetic jet and large missing transverse momentum using the ATLAS detector, J. High Energy Phys. 01 (2018) 126.

[16] J. M. Lindert et al., Precise predictions for $\mathrm{V}+$ jets dark matter backgrounds, Eur. Phys. J. C 77, 829 (2017).

[17] G. Busoni, A. De Simone, E. Morgante, and A. Riotto, On the validity of the effective field theory for dark matter searches at the LHC, Phys. Lett. B 728, 412 (2014).
[18] O. Buchmueller, M. J. Dolan, and C. McCabe, Beyond effective field theory for dark matter searches at the LHC, J. High Energy Phys. 01 (2014) 025.

[19] O. Buchmueller, M. J. Dolan, S. A. Malik, and C. McCabe, Characterising dark matter searches at colliders and direct detection experiments: Vector mediators, J. High Energy Phys. 01 (2015) 037.

[20] Y. Bai and J. Berger, Fermion portal dark matter, J. High Energy Phys. 11 (2013) 171.

[21] B. Dutta, Y. Gao, and T. Kamon, Probing light nonthermal dark matter at the LHC, Phys. Rev. D 89, 096009 (2014).

[22] R. Allahverdi and B. Dutta, Natural GeV dark matter and the baryon-dark matter coincidence puzzle, Phys. Rev. D 88, 023525 (2013).

[23] J. Alwall, P. C. Schuster, and N. Toro, Simplified models for a first characterization of new physics at the LHC, Phys. Rev. D 79, 075020 (2009).

[24] J. Goodman and W. Shepherd, LHC bounds on UVcomplete models of dark matter, arXiv:1111.2359.

[25] D. Alves (LHC New Physics Working Group), Simplified models for LHC new physics searches, J. Phys. G 39, 105005 (2012).

[26] H. An, X. Ji, and L.-T. Wang, Light dark matter and $Z^{\prime}$ dark force at colliders, J. High Energy Phys. 047 (2012) 182.

[27] H. An, R. Huo, and L.-T. Wang, Searching for low mass dark portal at the LHC, Phys. Dark Universe 2, 50 (2013).

[28] A. DiFranzo, K. I. Nagao, A. Rajaraman, and T. M. P. Tait, Simplified models for dark matter interacting with quarks, J. High Energy Phys. 11 (2013) 014; Erratum, 01 (2014) 162(E).

[29] Y. Bai and J. Berger, Lepton portal dark matter, J. High Energy Phys. 08 (2014) 153.

[30] H. An, L.-T. Wang, and H. Zhang, Dark matter with $t$-channel mediator: a simple step beyond contact interaction, Phys. Rev. D 89, 115014 (2014).

[31] J. Abdallah et al., Simplified models for dark matter and missing energy searches at the LHC, arXiv:1409.2893.

[32] S. A. Malik et al., Interplay and characterization of dark matter searches at colliders and in direct detection experiments, Phys. Dark Universe 9, 51 (2015).

[33] P. Harris, V. V. Khoze, M. Spannowsky, and C. Williams, Constraining dark sectors at colliders: Beyond the effective theory approach, Phys. Rev. D 91, 055009 (2015).

[34] M. R. Buckley, D. Feld, and D. Goncalves, Scalar simplified models for dark matter, Phys. Rev. D 91, 015017 (2015).

[35] U. Haisch and E. Re, Simplified dark matter top-quark interactions at the LHC, J. High Energy Phys. 06 (2015) 078.

[36] P. Harris, V. V. Khoze, M. Spannowsky, and C. Williams, Closing up on dark sectors at colliders: from 14 to $100 \mathrm{TeV}$, Phys. Rev. D 93, 054030 (2016).

[37] L. M. Carpenter, A. Nelson, C. Shimmin, T. M. P. Tait, and D. Whiteson, Collider searches for dark matter in events with a $\mathrm{Z}$ boson and missing energy, Phys. Rev. D 87, 074005 (2013).

[38] N. F. Bell, A. J. Galea, J. B. Dent, T. D. Jacques, L. M. Krauss, and T. J. Weiler, Searching for dark matter at the LHC with a mono-Z, Phys. Rev. D 86, 096011 (2012). 
[39] ATLAS Collaboration, Constraints on new phenomena via Higgs boson couplings and invisible decays with the ATLAS detector, J. High Energy Phys. 11 (2015) 206.

[40] CMS Collaboration, Searches for invisible decays of the Higgs boson in pp collisions at $\sqrt{s}=7,8$, and $13 \mathrm{TeV}, \mathrm{J}$. High Energy Phys. 02 (2017) 135.

[41] ATLAS Collaboration, Observation of a new particle in the search for the Standard Model Higgs boson with the ATLAS detector at the LHC, Phys. Lett. B 716, 1 (2012).

[42] CMS Collaboration, Observation of a new boson at a mass of $125 \mathrm{GeV}$ with the CMS experiment at the LHC, Phys. Lett. B 716, 30 (2012).

[43] CMS Collaboration, Observation of a new boson with mass near $125 \mathrm{GeV}$ in pp collisions at $\sqrt{s}=7$ and $8 \mathrm{TeV}$, J. High Energy Phys. 06 (2013) 081.

[44] CMS Collaboration, The CMS experiment at the CERN LHC, J. Instrum. 3, S08004 (2008).

[45] CMS Collaboration, Particle-flow reconstruction and global event description with the CMS detector, J. Instrum. 12, P10003 (2017).

[46] M. Cacciari, G. P. Salam, and G. Soyez, The anti- $k_{t}$ jet clustering algorithm, J. High Energy Phys. 04 (2008) 063.

[47] M. Cacciari, G. P. Salam, and G. Soyez, FastJet user manual, Eur. Phys. J. C 72, 1896 (2012).

[48] CMS Collaboration, Jet energy scale and resolution in the CMS experiment in pp collisions at $8 \mathrm{TeV}$, J. Instrum. 12, P02014 (2017).

[49] CMS Collaboration, CMS Physics Analysis Summary Report No. CMS-PAS-JME-16-004, https://cds.cern.ch/ record/2205284.

[50] CMS Collaboration, Performance of the CMS missing transverse momentum reconstruction in $\mathrm{pp}$ data at $\sqrt{s}=8 \mathrm{TeV}$, J. Instrum. 10, P02006 (2015).

[51] CMS Collaboration, Performance of CMS muon reconstruction in pp collision events at $\sqrt{s}=7 \mathrm{TeV}$, J. Instrum. 7, P10002 (2012).

[52] CMS Collaboration, Performance of electron reconstruction and selection with the CMS detector in proton-proton collisions at $\sqrt{s}=8 \mathrm{TeV}$, J. Instrum. 10, P06005 (2015).

[53] CMS Collaboration, Description and performance of track and primary-vertex reconstruction with the CMS tracker, J. Instrum. 9, P10009 (2014).

[54] CMS Collaboration, Performance of photon reconstruction and identification with the CMS detector in proton-proton collisions at $\sqrt{s}=8 \mathrm{TeV}$, J. Instrum. 10, P08010 (2015).

[55] CMS Collaboration, Reconstruction and identification of $\tau$ lepton decays to hadrons and $\nu_{\tau}$ at CMS, J. Instrum. 11, P01019 (2016).

[56] J. Alwall, R. Frederix, S. Frixione, V. Hirschi, F. Maltoni, O. Mattelaer, H.-S. Shao, T. Stelzer, P. Torrielli, and M. Zaro, The automated computation of tree-level and nextto-leading order differential cross sections, and their matching to parton shower simulations, J. High Energy Phys. 07 (2014) 079.

[57] C. Oleari, The POWHEG box, Nucl. Phys. B, Proc. Suppl. 205, 36 (2010).

[58] S. Alioli, P. Nason, C. Oleari, and E. Re, NLO single-top production matched with shower in POWHEG: $s$ - and $t$ channel contributions, J. High Energy Phys. 09 (2009) 111; Erratum, 02 (2010) 11(E).
[59] T. Sjöstrand, S. Ask, J. R. Christiansen, R. Corke, N. Desai, P. Ilten, S. Mrenna, S. Prestel, C. O. Rasmussen, and P.Z. Skands, An introduction to PYTHIA 8.2, Comput. Phys. Commun. 191, 159 (2015).

[60] M. Backović, M. Krämer, F. Maltoni, A. Martini, K. Mawatari, and M. Pellen, Higher-order QCD predictions for dark matter production at the LHC in simplified models with s-channel mediators, Eur. Phys. J. C 75, 482 (2015).

[61] O. Mattelaer and E. Vryonidou, Dark matter production through loop-induced processes at the LHC: The $s$-channel mediator case, Eur. Phys. J. C 75, 436 (2015).

[62] S. Alioli, P. Nason, C. Oleari, and E. Re, NLO Higgs boson production via gluon fusion matched with shower in POWHEG, J. High Energy Phys. 04 (2009) 002.

[63] P. Nason and C. Oleari, NLO Higgs boson production via vector-boson fusion matched with shower in POWHEG, J. High Energy Phys. 02 (2010) 037.

[64] I. Anderson et al., Constraining anomalous HVV interactions at proton and lepton colliders, Phys. Rev. D 89, 035007 (2014).

[65] CMS Collaboration, Event generator tunes obtained from underlying event and multiparton scattering measurements, Eur. Phys. J. C 76, 155 (2016).

[66] M. L. Mangano, M. Moretti, F. Piccinini, and M. Treccani, Matching matrix elements and shower evolution for topquark production in hadronic collisions, J. High Energy Phys. 01 (2007) 013.

[67] R. Frederix and S. Frixione, Merging meets matching in MC@NLO, J. High Energy Phys. 12 (2012) 061.

[68] R. D. Ball et al. (NNPDF Collaboration), Parton distributions for the LHC Run II, J. High Energy Phys. 04 (2015) 040.

[69] S. Agostinelli et al. (GEANT4 Collaboration), GEANT4 A simulation toolkit, Nucl. Instrum. Methods Phys. Res., Sect. A 506, 250 (2003).

[70] CMS Collaboration, Physics Analysis Summary Report No. CMS-PAS-LUM-17-001, http://cds.cern.ch/record/ 2257069.

[71] CMS Collaboration, Identification of b-quark jets with the CMS experiment, J. Instrum. 8, P04013 (2013).

[72] CMS Collaboration, CMS Physics Analysis Summary Report No. CMS-PAS-BTV-15-001, https://cds.cern.ch/ record/2138504.

[73] CMS Collaboration, CMS Physics Analysis Summary Report No. CMS-PAS-JME-16-003, http://cds.cern.ch/ record/2256875.

[74] S. D. Ellis, C. K. Vermilion, and J. R. Walsh, Recombination algorithms and jet substructure: Pruning as a tool for heavy particle searches, Phys. Rev. D 81, 094023 (2010).

[75] S. D. Ellis, C. K. Vermilion, and J. R. Walsh, Techniques for improved heavy particle searches with jet substructure, Phys. Rev. D 80, 051501 (2009).

[76] Yu. L. Dokshitzer, G. D. Leder, S. Moretti, and B. R. Webber, Better jet clustering algorithms, J. High Energy Phys. 08 (1997) 001.

[77] J. Thaler and K. Van Tilburg, Identifying boosted objects with N-subjettiness, J. High Energy Phys. 03 (2011) 015.

[78] A. Denner, S. Dittmaier, T. Kasprzik, and A. Muck, Electroweak corrections to $\mathrm{W}+$ jet hadroproduction including leptonic W-boson decays, J. High Energy Phys. 08 (2009) 075. 
[79] A. Denner, S. Dittmaier, T. Kasprzik, and A. Muck, Electroweak corrections to dilepton + jet production at hadron colliders, J. High Energy Phys. 06 (2011) 069.

[80] A. Denner, S. Dittmaier, T. Kasprzik, and A. Maeck, Electroweak corrections to monojet production at the LHC, Eur. Phys. J. C 73, 2297 (2013).

[81] J. H. Kuhn, A. Kulesza, S. Pozzorini, and M. Schulze, Electroweak corrections to hadronic photon production at large transverse momenta, J. High Energy Phys. 03 (2006) 059.

[82] S. Kallweit, J. M. Lindert, P. Maierhafer, S. Pozzorini, and M. Schanherr, NLO electroweak automation and precise predictions for $\mathrm{W}+$ multijet production at the LHC, J. High Energy Phys. 04 (2015) 012.

[83] S. Kallweit, J. M. Lindert, P. Maierhofer, S. Pozzorini, and M. Schanherr, NLO QCD + EW predictions for $\mathrm{V}+$ jets including off-shell vector-boson decays and multijet merging, J. High Energy Phys. 04 (2016) 021.

[84] CMS Collaboration, Search for new physics with jets and missing transverse momentum in $\mathrm{pp}$ collisions at $\sqrt{s}=7 \mathrm{TeV}$, J. High Energy Phys. 08 (2011) 155.

[85] J. H. Kuhn, A. Kulesza, S. Pozzorini, and M. Schulze, Logarithmic electroweak corrections to hadronic $Z+1$ jet production at large transverse momentum, Phys. Lett. B 609, 277 (2005).

[86] J. H. Kuhn, A. Kulesza, S. Pozzorini, and M. Schulze, Oneloop weak corrections to hadronic production of $\mathrm{Z}$ bosons at large transverse momenta, Nucl. Phys. B727, 368 (2005).

[87] J. H. Kuhn, A. Kulesza, S. Pozzorini, and M. Schulze, Electroweak corrections to hadronic production of W bosons at large transverse momenta, Nucl. Phys. B797, 27 (2008).

[88] CMS Collaboration, Measurement of differential cross sections for top quark pair production using the lepton + jets final state in proton-proton collisions at $13 \mathrm{TeV}$, Phys. Rev. D 95, 092001 (2017).

[89] CMS Collaboration, Measurement of the Top Quark Pair Production Cross Section in Proton-Proton Collisions at $\sqrt{s}=13$ TeV, Phys. Rev. Lett. 116, 052002 (2016).

[90] CMS Collaboration, Measurement of the $\mathrm{ZZ}$ production cross section and $Z \rightarrow \ell^{+} \ell^{-} \ell^{\prime+} \ell^{\prime-}$ branching fraction in $\mathrm{pp}$ collisions at $\sqrt{s}=13 \mathrm{TeV}$, Phys. Lett. B 763, 280 (2016).

[91] CMS Collaboration, Measurement of the WZ production cross section in pp collisions at $\sqrt{s}=13 \mathrm{TeV}$, Phys. Lett. B 766, 268 (2017).

[92] CMS Collaboration, CMS Note Report No. CMS-NOTE2017-001, https://cds.cern.ch/record/2242860.

[93] D. Abercrombie et al., Dark matter benchmark models for early LHC Run-2 searches: Report of the ATLAS/CMS dark matter forum, arXiv:1507.00966.

[94] T. Junk, Confidence level computation for combining searches with small statistics, Nucl. Instrum. Methods Phys. Res., Sect. A 434, 435 (1999).

[95] A. L. Read, Presentation of search results: The $\mathrm{CL}_{\mathrm{s}}$ technique, J. Phys. G 28, 2693 (2002).

[96] G. Cowan, K. Cranmer, E. Gross, and O. Vitells, Asymptotic formulae for likelihood-based tests of new physics, Eur. Phys. J. C 71, 1554 (2011); 73Erratum, 2501(E) (2013).
[97] P. A. R. Ade et al. (Planck Collaboration), Planck 2015 results. XIII. Cosmological parameters, Astron. Astrophys. 594, A13 (2016).

[98] M. Backović, K. Kong, and M. McCaskey, MadDM v.1.0: Computation of dark matter relic abundance using MadGraph5, Phys. Dark Universe 5, 18 (2014).

[99] P. A. R. Ade et al. (Planck Collaboration), Planck 2013 results. XVI. Cosmological parameters, Astron. Astrophys. 571, A16 (2014).

[100] G. Busoni et al., Recommendations on presenting LHC searches for missing transverse energy signals using simplified s-channel models of dark matter, arXiv: 1603.04156.

[101] M. Ackermann et al. (Fermi-LAT Collaboration), Searching for Dark Matter Annihilation from Milky Way Dwarf Spheroidal Galaxies with Six Years of Fermi Large Area Telescope Data, Phys. Rev. Lett. 115, 231301 (2015).

[102] R. Agnese et al. (SuperCDMS Collaboration), New Results from the Search for Low-Mass Weakly Interacting Massive Particles with the CDMS Low Ionization Threshold Experiment, Phys. Rev. Lett. 116, 071301 (2016).

[103] D. S. Akerib et al. (LUX Collaboration), Results from a Search for Dark Matter in the Complete LUX Exposure, Phys. Rev. Lett. 118, 021303 (2017).

[104] E. Aprile et al. (XENON Collaboration), First Dark Matter Search Results from the XENON1T Experiment, Phys. Rev. Lett. 119, 181301 (2017).

[105] X. Cui et al. (PandaX-II Collaboration), Dark Matter Results from 54-Ton-Day zexposure of PandaX-II Experiment, Phys. Rev. Lett. 119, 181302 (2017).

[106] G. Angloher et al. (CRESST Collaboration), Results on light dark matter particles with a low-threshold CRESST-II detector, Eur. Phys. J. C 76, 25 (2016).

[107] E. Behnke et al., Final results of the PICASSO dark matter search experiment, Astropart. Phys. 90, 85 (2017).

[108] C. Amole et al. (PICO Collaboration), Dark matter search results from the PICO-60 $\mathrm{CF}_{3} \mathrm{I}$ bubble chamber, Phys. Rev. D 93, 052014 (2016).

[109] M. G. Aartsen et al. (IceCube Collaboration), Improved limits on dark matter annihilation in the Sun with the 79string IceCube detector and implications for supersymmetry, J. Cosmal. Astropart Phys. 04 (2016) 022.

[110] K. Choi et al. (Super-Kamiokande Collaboration), Search for Neutrinos from Annihilation of Captured Low-Mass Dark Matter Particles in the Sun by Super-Kamiokande, Phys. Rev. Lett. 114, 141301 (2015).

[111] U. Haisch, F. Kahlhoefer, and J. Unwin, The impact of heavy-quark loops on LHC dark matter searches, J. High Energy Phys. 07 (2013) 125.

[112] A. Berlin, D. Hooper, and S. D. McDermott, Simplified dark matter models for the galactic center gamma-ray excess, Phys. Rev. D 89, 115022 (2014).

[113] J. R. Andersen et al. (LHC Higgs Cross Section Working Group Collaboration), Handbook of LHC Higgs cross sections: 3. Higgs properties (2013). 
A. M. Sirunyan, ${ }^{1}$ A. Tumasyan, ${ }^{1}$ W. Adam, ${ }^{2}$ F. Ambrogi, ${ }^{2}$ E. Asilar, ${ }^{2}$ T. Bergauer, ${ }^{2}$ J. Brandstetter, ${ }^{2}$ E. Brondolin, ${ }^{2}$ M. Dragicevic, ${ }^{2}$ J. Erö, ${ }^{2}$ A. Escalante Del Valle, ${ }^{2}$ M. Flechl, ${ }^{2}$ M. Friedl, ${ }^{2}$ R. Frühwirth, ${ }^{2, b}$ V. M. Ghete, ${ }^{2}$ J. Grossmann, ${ }^{2}$ J. Hrubec, ${ }^{2}$ M. Jeitler, ${ }^{2, b}$ A. König, ${ }^{2}$ N. Krammer, ${ }^{2}$ I. Krätschmer, ${ }^{2}$ D. Liko, ${ }^{2}$ T. Madlener, ${ }^{2}$ I. Mikulec, ${ }^{2}$ E. Pree, ${ }^{2}$ N. Rad, ${ }^{2}$ H. Rohringer, ${ }^{2}$ J. Schieck, ${ }^{2, b}$ R. Schöfbeck, ${ }^{2}$ M. Spanring, ${ }^{2}$ D. Spitzbart, ${ }^{2}$ A. Taurok, ${ }^{2}$ W. Waltenberger, ${ }^{2}$ J. Wittmann, ${ }^{2}$ C.-E. Wulz ${ }^{2, b}$ M. Zarucki, ${ }^{2}$ V. Chekhovsky, ${ }^{3}$ V. Mossolov, ${ }^{3}$ J. Suarez Gonzalez, ${ }^{3}$ E. A. De Wolf, ${ }^{4}$ D. Di Croce, ${ }^{4}$ X. Janssen, ${ }^{4}$ J. Lauwers, ${ }^{4}$ M. Van De Klundert, ${ }^{4}$ H. Van Haevermaet, ${ }^{4}$ P. Van Mechelen, ${ }^{4}$ N. Van Remortel, ${ }^{4}$ S. Abu Zeid, ${ }^{5}$ F. Blekman, ${ }^{5}$ J. D’Hondt, ${ }^{5}$ I. De Bruyn, ${ }^{5}$ J. De Clercq, ${ }^{5}$ K. Deroover, ${ }^{5}$ G. Flouris, ${ }^{5}$ D. Lontkovskyi, ${ }^{5}$ S. Lowette, ${ }^{5}$ I. Marchesini, ${ }^{5}$ S. Moortgat,${ }^{5}$ L. Moreels, ${ }^{5}$ Q. Python, ${ }^{5}$ K. Skovpen, ${ }^{5}$ S. Tavernier, ${ }^{5}$ W. Van Doninck, ${ }^{5}$ P. Van Mulders, ${ }^{5}$ I. Van Parijs, ${ }^{5}$ D. Beghin, ${ }^{6}$ B. Bilin, ${ }^{6}$ H. Brun, ${ }^{6}$ B. Clerbaux,${ }^{6}$ G. De Lentdecker, ${ }^{6}$ H. Delannoy, ${ }^{6}$ B. Dorney, ${ }^{6}$ G. Fasanella, ${ }^{6}$ L. Favart, ${ }^{6}$ R. Goldouzian, ${ }^{6}$ A. Grebenyuk, ${ }^{6}$ A. K. Kalsi, ${ }^{6}$ T. Lenzi, ${ }^{6}$ J. Luetic, ${ }^{6}$ T. Maerschalk, ${ }^{6}$ A. Marinov, ${ }^{6}$ T. Seva, ${ }^{6}$ E. Starling, ${ }^{6}$ C. Vander Velde, ${ }^{6}$ P. Vanlaer, ${ }^{6}$ D. Vannerom, ${ }^{6}$ R. Yonamine, ${ }^{6}$ F. Zenoni, ${ }^{6}$ T. Cornelis, ${ }^{7}$ D. Dobur, ${ }^{7}$ A. Fagot, ${ }^{7}$ M. Gul, ${ }^{7}$ I. Khvastunov, ${ }^{7, \mathrm{c}}$ D. Poyraz, ${ }^{7}$ C. Roskas, ${ }^{7}$ S. Salva, ${ }^{7}$ M. Tytgat, ${ }^{7}$ W. Verbeke, ${ }^{7}$ N. Zaganidis, ${ }^{7}$ H. Bakhshiansohi, ${ }^{8}$ O. Bondu, ${ }^{8}$ S. Brochet, ${ }^{8}$ G. Bruno, ${ }^{8}$ C. Caputo, ${ }^{8}$ A. Caudron, ${ }^{8}$ P. David, ${ }^{8}$ S. De Visscher,${ }^{8}$ C. Delaere,${ }^{8}$ M. Delcourt, ${ }^{8}$ B. Francois,${ }^{8}$ A. Giammanco, ${ }^{8}$ M. Komm, ${ }^{8}$ G. Krintiras, ${ }^{8}$ V. Lemaitre, ${ }^{8}$ A. Magitteri, ${ }^{8}$ A. Mertens, ${ }^{8}$ M. Musich, ${ }^{8}$ K. Piotrzkowski, ${ }^{8}$ L. Quertenmont, ${ }^{8}$ A. Saggio, ${ }^{8}$ M. Vidal Marono, ${ }^{8}$ S. Wertz, ${ }^{8}$ J. Zobec, ${ }^{8}$ W. L. Aldá Júnior, ${ }^{9}$ F. L. Alves, ${ }^{9}$ G. A. Alves, ${ }^{9}$ L. Brito, ${ }^{9}$ M. Correa Martins Junior, ${ }^{9}$ G. Correia Silva, ${ }^{9}$ C. Hensel, ${ }^{9}$ A. Moraes, ${ }^{9}$ M. E. Pol, ${ }^{9}$ P. Rebello Teles, ${ }^{9}$ E. Belchior Batista Das Chagas, ${ }^{10}$ W. Carvalho, ${ }^{10}$ J. Chinellato, ${ }^{10, d}$ E. Coelho, ${ }^{10}$ E. M. Da Costa, ${ }^{10}$ G. G. Da Silveira,,${ }^{10, e}$ D. De Jesus Damiao, ${ }^{10}$ S. Fonseca De Souza, ${ }^{10}$ L. M. Huertas Guativa, ${ }^{10}$ H. Malbouisson, ${ }^{10}$ M. Melo De Almeida, ${ }^{10}$ C. Mora Herrera, ${ }^{10}$ L. Mundim, ${ }^{10}$ H. Nogima, ${ }^{10}$ L. J. Sanchez Rosas, ${ }^{10}$ A. Santoro, ${ }^{10}$ A. Sznajder,${ }^{10}$ M. Thiel,${ }^{10}$ E. J. Tonelli Manganote, ${ }^{10, \mathrm{~d}}$ F. Torres Da Silva De Araujo, ${ }^{10}$ A. Vilela Pereira, ${ }^{10}$ S. Ahuja, ${ }^{11 \mathrm{a}}$ C. A. Bernardes,${ }^{11 \mathrm{a}}$ T. R. Fernandez Perez Tomei, ${ }^{11 \mathrm{a}}$ E. M. Gregores, ${ }^{11 \mathrm{~b}}$ P. G. Mercadante, ${ }^{11 \mathrm{~b}}$ S. F. Novaes, ${ }^{11 \mathrm{a}}$ Sandra S. Padula, ${ }^{11 \mathrm{a}}$ D. Romero Abad, ${ }^{11 \mathrm{~b}}$ J. C. Ruiz Vargas, ${ }^{11 \mathrm{a}}$ A. Aleksandrov,${ }^{12}$ R. Hadjiiska, ${ }^{12}$ P. Iaydjiev, ${ }^{12}$ M. Misheva, ${ }^{12}$ M. Rodozov ${ }^{12}$ M. Shopova, ${ }^{12}$ G. Sultanov, ${ }^{12}$ A. Dimitrov, ${ }^{13}$ L. Litov ${ }^{13}$ B. Pavlov, ${ }^{13}$ P. Petkov, ${ }^{13}$ W. Fang, ${ }^{14, f}$ X. Gao, ${ }^{14, f}$ L. Yuan, ${ }^{14}$ M. Ahmad, ${ }^{15}$ J. G. Bian, ${ }^{15}$ G. M. Chen, ${ }^{15}$ H. S. Chen,${ }^{15}$ M. Chen, ${ }^{15}$ Y. Chen,${ }^{15}$ C. H. Jiang, ${ }^{15}$ D. Leggat, ${ }^{15}$ H. Liao, ${ }^{15}$ Z. Liu, ${ }^{15}$ F. Romeo, ${ }^{15}$ S. M. Shaheen, ${ }^{15}$ A. Spiezia, ${ }^{15}$ J. Tao, ${ }^{15}$ C. Wang, ${ }^{15}$ Z. Wang, ${ }^{15}$ E. Yazgan, ${ }^{15}$ T. Yu, ${ }^{15}$ H. Zhang, ${ }^{15}$ S. Zhang, ${ }^{15}$ J. Zhao, ${ }^{15}$ Y. Ban, ${ }^{16}$ G. Chen, ${ }^{16}$ J. Li,${ }^{16}$ Q. Li, ${ }^{16}$ S. Liu,${ }^{16}$ Y. Mao, ${ }^{16}$ S. J. Qian, ${ }^{16}$ D. Wang, ${ }^{16}$ Z. Xu, ${ }^{16}$ F. Zhang, ${ }^{16, f}$ Y. Wang, ${ }^{17}$ C. Avila, ${ }^{18}$ A. Cabrera ${ }^{18}$ C. A. Carrillo Montoya, ${ }^{18}$ L. F. Chaparro Sierra, ${ }^{18}$ C. Florez, ${ }^{18}$ C. F. González Hernández, ${ }^{18}$ J. D. Ruiz Alvarez, ${ }^{18}$ M. A. Segura Delgado, ${ }^{18}$ B. Courbon, ${ }^{19}$ N. Godinovic,${ }^{19}$ D. Lelas, ${ }^{19}$ I. Puljak,${ }^{19}$ P. M. Ribeiro Cipriano, ${ }^{19}$ T. Sculac, ${ }^{19}$ Z. Antunovic, ${ }^{20}$ M. Kovac,${ }^{20}$ V. Brigljevic,${ }^{21}$ D. Ferencek, ${ }^{21}$ K. Kadija,${ }^{21}$ B. Mesic, ${ }^{21}$ A. Starodumov, ${ }^{21, g}$ T. Susa, ${ }^{21}$ M. W. Ather, ${ }^{22}$ A. Attikis, ${ }^{22}$ G. Mavromanolakis, ${ }^{22}$ J. Mousa, ${ }^{22}$ C. Nicolaou, ${ }^{22}$ F. Ptochos, ${ }^{22}$ P. A. Razis, ${ }^{22}$ H. Rykaczewski, ${ }^{22}$ M. Finger, ${ }^{23, h}$ M. Finger Jr., ${ }^{23, h}$ E. Carrera Jarrin ${ }^{24}$ Y. Assran, ${ }^{25, i, j}$ S. Elgammal, ${ }^{25, j}$ S. Khalil, ${ }^{25, k}$ S. Bhowmik, ${ }^{26}$ R. K. Dewanjee, ${ }^{26}$ M. Kadastik, ${ }^{26}$ L. Perrini, ${ }^{26}$ M. Raidal, ${ }^{26}$ A. Tiko, ${ }^{26}$ C. Veelken, ${ }^{26}$ P. Eerola, ${ }^{27}$ H. Kirschenmann, ${ }^{27}$ J. Pekkanen, ${ }^{27}$ M. Voutilainen, ${ }^{27}$ J. Havukainen, ${ }^{28}$ J. K. Heikkilä, ${ }^{28}$ T. Järvinen, ${ }^{28}$ V. Karimäki, ${ }^{28}$ R. Kinnunen, ${ }^{28}$ T. Lampén, ${ }^{28}$ K. Lassila-Perini, ${ }^{28}$ S. Laurila, ${ }^{28}$ S. Lehti, ${ }^{28}$ T. Lindén, ${ }^{28}$ P. Luukka, ${ }^{28}$ T. Mäenpää, ${ }^{28}$ H. Siikonen, ${ }^{28}$ E. Tuominen, ${ }^{28}$ J. Tuominiemi, ${ }^{28}$ T. Tuuva, ${ }^{29}$ M. Besancon, ${ }^{30}$ F. Couderc, ${ }^{30}$ M. Dejardin, ${ }^{30}$ D. Denegri, ${ }^{30}$ J. L. Faure ${ }^{30}$ F. Ferri, ${ }^{30}$ S. Ganjour, ${ }^{30}$ S. Ghosh ${ }^{30}$ A. Givernaud, ${ }^{30}$ P. Gras, ${ }^{30}$ G. Hamel de Monchenault, ${ }^{30}$ P. Jarry, ${ }^{30}$ I. Kucher,${ }^{30}$ C. Leloup,${ }^{30}$ E. Locci ${ }^{30}$ M. Machet, ${ }^{30}$ J. Malcles, ${ }^{30}$ G. Negro,${ }^{30}$ J. Rander, ${ }^{30}$ A. Rosowsky, ${ }^{30}$ M. Ö. Sahin, ${ }^{30}$ M. Titov ${ }^{30}$ A. Abdulsalam, ${ }^{31,1}$ C. Amendola,${ }^{31}$ I. Antropov, ${ }^{31}$ S. Baffioni,${ }^{31}$ F. Beaudette,${ }^{31}$ P. Busson ${ }^{31}$ L. Cadamuro, ${ }^{31}$

C. Charlot, ${ }^{31}$ R. Granier de Cassagnac, ${ }^{31}$ M. Jo, ${ }^{31}$ S. Lisniak, ${ }^{31}$ A. Lobanov, ${ }^{31}$ J. Martin Blanco, ${ }^{31}$ M. Nguyen, ${ }^{31}$

C. Ochando, ${ }^{31}$ G. Ortona ${ }^{31}$ P. Paganini,${ }^{31}$ P. Pigard,${ }^{31}$ R. Salerno, ${ }^{31}$ J. B. Sauvan, ${ }^{31}$ Y. Sirois, ${ }^{31}$ A. G. Stahl Leiton, ${ }^{31}$ T. Strebler, ${ }^{31}$ Y. Yilmaz, ${ }^{31}$ A. Zabi, ${ }^{31}$ A. Zghiche, ${ }^{31}$ J.-L. Agram, ${ }^{32, m}$ J. Andrea, ${ }^{32}$ D. Bloch, ${ }^{32}$ J.-M. Brom, ${ }^{32}$ M. Buttignol, ${ }^{32}$ E. C. Chabert, ${ }^{32}$ N. Chanon, ${ }^{32}$ C. Collard, ${ }^{32}$ E. Conte, ${ }^{32, \mathrm{~m}}$ X. Coubez, ${ }^{32}$ F. Drouhin, ${ }^{32, \mathrm{~m}}$ J.-C. Fontaine, ${ }^{32, \mathrm{~m}}$ D. Gelé, ${ }^{32}$ U. Goerlach, ${ }^{32}$ M. Jansová, ${ }^{32}$ P. Juillot ${ }^{32}$ A.-C. Le Bihan, ${ }^{32}$ N. Tonon, ${ }^{32}$ P. Van Hove, ${ }^{32}$ S. Gadrat, ${ }^{33}$ S. Beauceron, ${ }^{34}$ C. Bernet, ${ }^{34}$ G. Boudoul,${ }^{34}$ R. Chierici, ${ }^{34}$ D. Contardo, ${ }^{34}$ P. Depasse,${ }^{34}$ H. El Mamouni, ${ }^{34}$ J. Fay,${ }^{34}$ L. Finco, ${ }^{34}$ S. Gascon, ${ }^{34}$ M. Gouzevitch, ${ }^{34}$ G. Grenier, ${ }^{34}$ B. Ille, ${ }^{34}$ F. Lagarde,${ }^{34}$ I. B. Laktineh, ${ }^{34}$ M. Lethuillier, ${ }^{34}$ L. Mirabito, ${ }^{34}$ A. L. Pequegnot,${ }^{34}$

S. Perries, ${ }^{34}$ A. Popov, ${ }^{34, n}$ V. Sordini,${ }^{34}$ M. Vander Donckt, ${ }^{34}$ S. Viret, ${ }^{34}$ T. Toriashvili, ${ }^{35,0}$ Z. Tsamalaidze, ${ }^{36, h}$

C. Autermann, ${ }^{37}$ L. Feld ${ }^{37}$ M. K. Kiesel, ${ }^{37}$ K. Klein, ${ }^{37}$ M. Lipinski, ${ }^{37}$ M. Preuten, ${ }^{37}$ C. Schomakers, ${ }^{37}$ J. Schulz, ${ }^{37}$ M. Teroerde,${ }^{37}$ B. Wittmer, ${ }^{37}$ V. Zhukov, ${ }^{37, \mathrm{n}}$ A. Albert ${ }^{38}$ D. Duchardt, ${ }^{38}$ M. Endres,${ }^{38}$ M. Erdmann,${ }^{38}$ S. Erdweg, ${ }^{38}$ T. Esch, ${ }^{38}$ R. Fischer, ${ }^{38}$ A. Güth, ${ }^{38}$ M. Hamer, ${ }^{38}$ T. Hebbeker, ${ }^{38}$ C. Heidemann, ${ }^{38}$ K. Hoepfner, ${ }^{38}$ S. Knutzen, ${ }^{38}$ M. Merschmeyer, ${ }^{38}$ 
A. Meyer, ${ }^{38}$ P. Millet, ${ }^{38}$ S. Mukherjee, ${ }^{38}$ T. Pook,${ }^{38}$ M. Radziej, ${ }^{38}$ H. Reithler ${ }^{38}$ M. Rieger, ${ }^{38}$ F. Scheuch,${ }^{38}$ D. Teyssier, ${ }^{38}$ S. Thüer, ${ }^{38}$ G. Flügge, ${ }^{39}$ B. Kargoll,${ }^{39}$ T. Kress, ${ }^{39}$ A. Künsken, ${ }^{39}$ T. Müller, ${ }^{39}$ A. Nehrkorn, ${ }^{39}$ A. Nowack,${ }^{39}$ C. Pistone, ${ }^{39}$ O. Pooth, ${ }^{39}$ A. Stahl, ${ }^{39, p}$ M. Aldaya Martin, ${ }^{40}$ T. Arndt, ${ }^{40}$ C. Asawatangtrakuldee, ${ }^{40}$ K. Beernaert, ${ }^{40}$ O. Behnke, ${ }^{40}$ U. Behrens, ${ }^{40}$ A. Bermúdez Martínez, ${ }^{40}$ A. A. Bin Anuar, ${ }^{40}$ K. Borras,${ }^{40, q}$ V. Botta, ${ }^{40}$ A. Campbell, ${ }^{40}$ P. Connor, ${ }^{40}$ C. Contreras-Campana,${ }^{40}$ F. Costanza, ${ }^{40}$ C. Diez Pardos ${ }^{40}$ G. Eckerlin, ${ }^{40}$ D. Eckstein, ${ }^{40}$ T. Eichhorn, ${ }^{40}$ E. Eren, ${ }^{40}$ E. Gallo, ${ }^{40, r}$ J. Garay Garcia, ${ }^{40}$ A. Geiser, ${ }^{40}$ J. M. Grados Luyando, ${ }^{40}$ A. Grohsjean, ${ }^{40}$ P. Gunnellini, ${ }^{40}$ M. Guthoff, ${ }^{40}$ A. Harb, ${ }^{40}$ J. Hauk, ${ }^{40}$ M. Hempel, ${ }^{40, s}$ H. Jung, ${ }^{40}$ M. Kasemann, ${ }^{40}$ J. Keaveney,${ }^{40}$ C. Kleinwort, ${ }^{40}$ I. Korol, ${ }^{40}$ D. Krücker,${ }^{40}$ W. Lange, ${ }^{40}$ A. Lelek, ${ }^{40}$ T. Lenz, ${ }^{40}$ J. Leonard, ${ }^{40}$ K. Lipka, ${ }^{40}$ W. Lohmann, ${ }^{40, s}$ R. Mankel, ${ }^{40}$ I.-A. Melzer-Pellmann, ${ }^{40}$

A. B. Meyer, ${ }^{40}$ G. Mittag, ${ }^{40}$ J. Mnich, ${ }^{40}$ A. Mussgiller, ${ }^{40}$ E. Ntomari, ${ }^{40}$ D. Pitzl,${ }^{40}$ A. Raspereza,${ }^{40}$ M. Savitskyi, ${ }^{40}$ P. Saxena, ${ }^{40}$ R. Shevchenko, ${ }^{40}$ N. Stefaniuk, ${ }^{40}$ G. P. Van Onsem, ${ }^{40}$ R. Walsh, ${ }^{40}$ Y. Wen, ${ }^{40}$ K. Wichmann, ${ }^{40}$ C. Wissing, ${ }^{40}$ O. Zenaiev, ${ }^{40}$ R. Aggleton, ${ }^{41}$ S. Bein, ${ }^{41}$ V. Blobel, ${ }^{41}$ M. Centis Vignali, ${ }^{41}$ T. Dreyer, ${ }^{41}$ E. Garutti, ${ }^{41}$ D. Gonzalez, ${ }^{41}$ J. Haller, ${ }^{41}$ A. Hinzmann, ${ }^{41}$ M. Hoffmann, ${ }^{41}$ A. Karavdina, ${ }^{41}$ R. Klanner ${ }^{41}$ R. Kogler ${ }^{41}$ N. Kovalchuk, ${ }^{41}$ S. Kurz, ${ }^{41}$ T. Lapsien, ${ }^{41}$ D. Marconi, ${ }^{41}$ M. Meyer, ${ }^{41}$ M. Niedziela,${ }^{41}$ D. Nowatschin, ${ }^{41}$ F. Pantaleo, ${ }^{41, p}$ T. Peiffer, ${ }^{41}$ A. Perieanu, ${ }^{41}$ C. Scharf ${ }^{41}$ P. Schleper, ${ }^{41}$ A. Schmidt, ${ }^{41}$ S. Schumann, ${ }^{41}$ J. Schwandt, ${ }^{41}$ J. Sonneveld, ${ }^{41}$ H. Stadie, ${ }^{41}$ G. Steinbrück, ${ }^{41}$ F. M. Stober, ${ }^{41}$ M. Stöver ${ }^{41}$ H. Tholen, ${ }^{41}$ D. Troendle, ${ }^{41}$ E. Usai, ${ }^{41}$ A. Vanhoefer, ${ }^{41}$ B. Vormwald, ${ }^{41}$ M. Akbiyik, ${ }^{42}$ C. Barth,${ }^{42}$ M. Baselga ${ }^{42}$ S. Baur, ${ }^{42}$ E. Butz,${ }^{42}$ R. Caspart, ${ }^{42}$ T. Chwalek, ${ }^{42}$ F. Colombo, ${ }^{42}$ W. De Boer, ${ }^{42}$ A. Dierlamm, ${ }^{42}$ N. Faltermann,${ }^{42}$ B. Freund, ${ }^{42}$ R. Friese, ${ }^{42}$ M. Giffels, ${ }^{42}$ M. A. Harrendorf ${ }^{42}$ F. Hartmann, ${ }^{42, p}$ S. M. Heindl, ${ }^{42}$ U. Husemann, ${ }^{42}$ F. Kassel, ${ }^{42, p}$ S. Kudella, ${ }^{42}$ H. Mildner, ${ }^{42}$ M. U. Mozer, ${ }^{42}$ Th. Müller, ${ }^{42}$ M. Plagge, ${ }^{42}$ G. Quast ${ }^{42}$ K. Rabbertz, ${ }^{42}$ M. Schröder ${ }^{42}$ I. Shvetsov, ${ }^{42}$ G. Sieber, ${ }^{42}$ H. J. Simonis, ${ }^{42}$ R. Ulrich ${ }^{42}$ S. Wayand,${ }^{42}$ M. Weber ${ }^{42}$ T. Weiler, ${ }^{42}$ S. Williamson, ${ }^{42}$ C. Wöhrmann, ${ }^{42}$ R. Wolf, ${ }^{42}$ G. Anagnostou, ${ }^{43}$ G. Daskalakis, ${ }^{43}$ T. Geralis ${ }^{43}$ A. Kyriakis, ${ }^{43}$ D. Loukas, ${ }^{43}$ I. Topsis-Giotis, ${ }^{43}$ G. Karathanasis ${ }^{44}$ S. Kesisoglou, ${ }^{44}$ A. Panagiotou, ${ }^{44}$ N. Saoulidou, ${ }^{44}$ K. Kousouris, ${ }^{45}$ I. Evangelou, ${ }^{46}$ C. Foudas, ${ }^{46}$ P. Gianneios,${ }^{46}$

P. Katsoulis, ${ }^{46}$ P. Kokkas, ${ }^{46}$ S. Mallios, ${ }^{46}$ N. Manthos, ${ }^{46}$ I. Papadopoulos, ${ }^{46}$ E. Paradas, ${ }^{46}$ J. Strologas,${ }^{46}$ F. A. Triantis ${ }^{46}$ D. Tsitsonis, ${ }^{46}$ M. Csanad, ${ }^{47}$ N. Filipovic, ${ }^{47}$ G. Pasztor,${ }^{47}$ O. Surányi, ${ }^{47}$ G. I. Veres,${ }^{47, t}$ G. Bencze,${ }^{48}$ C. Hajdu, ${ }^{48}$ D. Horvath, ${ }^{48, u}$ Á. Hunyadi, ${ }^{48}$ F. Sikler, ${ }^{48}$ V. Veszpremi ${ }^{48}$ G. Vesztergombi,${ }^{48, t}$ N. Beni, ${ }^{49}$ S. Czellar, ${ }^{49}$ J. Karancsi, ${ }^{49, v}$ A. Makovec, ${ }^{49}$ J. Molnar, ${ }^{49}$ Z. Szillasi, ${ }^{49}$ M. Bartók,${ }^{50, t}$ P. Raics, ${ }^{50}$ Z. L. Trocsanyi, ${ }^{50}$ B. Ujvari, ${ }^{50}$ S. Choudhury, ${ }^{51}$ J. R. Komaragiri, ${ }^{51}$ S. Bahinipati, ${ }^{52, w}$ P. Mal, ${ }^{52}$ K. Mandal, ${ }^{52}$ A. Nayak, ${ }^{52, \mathrm{x}}$ D. K. Sahoo, ${ }^{52, w}$ N. Sahoo, ${ }^{52}$ S. K. Swain, ${ }^{52}$ S. Bansal,${ }^{53}$ S. B. Beri,${ }^{53}$ V. Bhatnagar,${ }^{53}$ R. Chawla, ${ }^{53}$ N. Dhingra, ${ }^{53}$ A. Kaur,${ }^{53}$ M. Kaur,${ }^{53}$ S. Kaur,${ }^{53}$ R. Kumar,${ }^{53}$ P. Kumari, ${ }^{53}$ A. Mehta, ${ }^{53}$ J. B. Singh ${ }^{53}$ G. Walia, ${ }^{53}$ Ashok Kumar, ${ }^{54}$ Aashaq Shah, ${ }^{54}$ A. Bhardwaj, ${ }^{54}$ S. Chauhan, ${ }^{54}$ B. C. Choudhary, ${ }^{54}$ R. B. Garg, ${ }^{54}$ S. Keshri, ${ }^{54}$ A. Kumar, ${ }^{54}$ S. Malhotra,${ }^{54}$ M. Naimuddin, ${ }^{54}$ K. Ranjan,${ }^{54}$ R. Sharma,${ }^{54}$ R. Bhardwaj, ${ }^{55}$ R. Bhattacharya, ${ }^{55}$ S. Bhattacharya, ${ }^{55}$ U. Bhawandeep, ${ }^{55}$ S. Dey, ${ }^{55}$ S. Dutt, ${ }^{55}$ S. Dutta, ${ }^{55}$ S. Ghosh, ${ }^{55}$ N. Majumdar, ${ }^{55}$ A. Modak, ${ }^{55}$ K. Mondal,${ }^{55}$ S. Mukhopadhyay, ${ }^{55}$ S. Nandan, ${ }^{55}$ A. Purohit, ${ }^{55}$ A. Roy, ${ }^{55}$ S. Roy Chowdhury,${ }^{55}$ S. Sarkar, ${ }^{55}$ M. Sharan, ${ }^{55}$ S. Thakur, ${ }^{55}$ P. K. Behera, ${ }^{56}$ R. Chudasama, ${ }^{57}$ D. Dutta ${ }^{57}$ V. Jha, ${ }^{57}$ V. Kumar,${ }^{57}$ A. K. Mohanty, ${ }^{57, p}$ P. K. Netrakanti, ${ }^{57}$ L. M. Pant,${ }^{57}$ P. Shukla,${ }^{57}$ A. Topkar,${ }^{57}$ T. Aziz,${ }^{58}$ S. Dugad,${ }^{58}$ B. Mahakud,${ }^{58}$ S. Mitra, ${ }^{58}$ G. B. Mohanty,${ }^{58}$ N. Sur, ${ }^{58}$ B. Sutar, ${ }^{58}$ S. Banerjee, ${ }^{59}$ S. Bhattacharya, ${ }^{59}$ S. Chatterjee, ${ }^{59}$ P. Das,${ }^{59}$ M. Guchait, ${ }^{59}$ Sa. Jain, ${ }^{59}$ S. Kumar, ${ }^{59}$ M. Maity, ${ }^{59, y}$ G. Majumder, ${ }^{59}$ K. Mazumdar, ${ }^{59}$ T. Sarkar,${ }^{59, y}$ N. Wickramage,${ }^{59, z}$ S. Chauhan, ${ }^{60}$ S. Dube, ${ }^{60}$ V. Hegde, ${ }^{60}$

A. Kapoor, ${ }^{60}$ K. Kothekar, ${ }^{60}$ S. Pandey, ${ }^{60}$ A. Rane, ${ }^{60}$ S. Sharma ${ }^{60}$ S. Chenarani, ${ }^{61, a a}$ E. Eskandari Tadavani, ${ }^{61}$

S. M. Etesami, ${ }^{61, a a}$ M. Khakzad, ${ }^{61}$ M. Mohammadi Najafabadi, ${ }^{61}$ M. Naseri, ${ }^{61}$ S. Paktinat Mehdiabadi, ${ }^{61, b b}$

F. Rezaei Hosseinabadi, ${ }^{61}$ B. Safarzadeh, ${ }^{61, c c}$ M. Zeinali, ${ }^{61}$ M. Felcini, ${ }^{62}$ M. Grunewald, ${ }^{62}$ M. Abbrescia, ${ }^{63 a, 63 b}$ C. Calabria ${ }^{63 a, 63 b}$ A. Colaleo, ${ }^{63 a}$ D. Creanza, ${ }^{63 a, 63 c}$ L. Cristella, ${ }^{63 a, 63 b}$ N. De Filippis, ${ }^{63 a, 63 c}$ M. De Palma ${ }^{63 a, 63 b}$ F. Errico, ${ }^{63 a, 63 b}$ L. Fiore, ${ }^{63 a}$ G. Iaselli, ${ }^{63 a, 63 c}$ S. Lezki, ${ }^{63 a, 63 b}$ G. Maggi, ${ }^{63 a, 63 c}$ M. Maggi, ${ }^{63 a}$ G. Miniello, ${ }^{63 a, 63 b}$ S. My, ${ }^{63 a, 63 b}$ S. Nuzzo, ${ }^{63 a, 63 b}$ A. Pompili, ${ }^{63 a, 63 b}$ G. Pugliese, ${ }^{63 a, 63 c}$ R. Radogna, ${ }^{63 a}$ A. Ranieri, ${ }^{63 a}$ G. Selvaggi, ${ }^{63 a, 63 b}$ A. Sharma, ${ }^{63 a}$ L. Silvestris, ${ }^{63 a, p}$

R. Venditti, ${ }^{63 a}$ P. Verwilligen, ${ }^{63 a}$ G. Abbiendi, ${ }^{64 a}$ C. Battilana,${ }^{64 a, 64 b}$ D. Bonacorsi, ${ }^{64 a, 64 b}$ L. Borgonovi,${ }^{64 a, 64 b}$

S. Braibant-Giacomelli, ${ }^{64 a, 64 b}$ R. Campanini, ${ }^{64 a, 64 b}$ P. Capiluppi, ${ }^{64 a, 64 b}$ A. Castro, ${ }^{64 a, 64 b}$ F. R. Cavallo, ${ }^{64 a}$ S. S. Chhibra,${ }^{64 a, 64 b}$ G. Codispoti, ${ }^{64 a, 64 b}$ M. Cuffiani, ${ }^{64 a, 64 b}$ G. M. Dallavalle, ${ }^{64 a}$ F. Fabbri, ${ }^{64 a}$ A. Fanfani, ${ }^{64 a, 64 b}$ D. Fasanella, ${ }^{64 a, 64 b}$ P. Giacomelli, ${ }^{64 a}$ C. Grandi, ${ }^{64 a}$ L. Guiducci, ${ }^{64 a, 64 b}$ S. Marcellini, ${ }^{64 a}$ G. Masetti, ${ }^{64 a}$ A. Montanari, ${ }^{64 a}$ F. L. Navarria, ${ }^{64 a, 64 b}$ A. Perrotta ${ }^{64 a}$ A. M. Rossi, ${ }^{64 a, 64 b}$ T. Rovelli, ${ }^{64 a, 64 b}$ G. P. Siroli, ${ }^{64 a, 64 b}$ N. Tosi, ${ }^{64 a}$ S. Albergo,${ }^{65 a, 65 b}$ S. Costa ${ }^{65 a, 65 b}$ A. Di Mattia, ${ }^{65 a}$ F. Giordano, ${ }^{65 a, 65 b}$ R. Potenza ${ }^{65 a, 65 b}$ A. Tricomi ${ }^{65 a, 65 b}$ C. Tuve, ${ }^{65 a, 65 b}$ G. Barbagli, ${ }^{66 a}$ K. Chatterjee, ${ }^{66 a, 66 b}$ V. Ciulli, ${ }^{66 a, 66 b}$ C. Civinini, ${ }^{66 a}$ R. D’Alessandro, ${ }^{66 a, 66 b}$ E. Focardi, ${ }^{66 a, 66 b}$ P. Lenzi, ${ }^{66 a, 66 b}$ M. Meschini, ${ }^{66 a}$ S. Paoletti, ${ }^{66 a}$ L. Russo, ${ }^{66 a, d d}$ G. Sguazzoni, ${ }^{66 a}$ D. Strom, ${ }^{66 a}$ L. Viliani, ${ }^{66 a}$ L. Benussi, ${ }^{67}$ S. Bianco,${ }^{67}$ F. Fabbri, ${ }^{67}$ D. Piccolo, ${ }^{67}$ 
F. Primavera, ${ }^{67, p}$ V. Calvelli, ${ }^{68,68 b}$ F. Ferro, ${ }^{68 a}$ F. Ravera, ${ }^{68 a, 68 b}$ E. Robutti, ${ }^{68 a}$ S. Tosi, ${ }^{68 a, 68 b}$ A. Benaglia, ${ }^{69 a}$ A. Beschi, ${ }^{69 b}$

L. Brianza, ${ }^{69 a, 69 b}$ F. Brivio, ${ }^{69 a, 69 b}$ V. Ciriolo, ${ }^{69 a, 69 b, p}$ M. E. Dinardo, ${ }^{69 a, 69 b}$ S. Fiorendi, ${ }^{69 a, 69 b}$ S. Gennai, ${ }^{69 a}$ A. Ghezzi, ${ }^{69 a, 69 b}$ P. Govoni, ${ }^{69 a, 69 b}$ M. Malberti, ${ }^{69 a, 69 b}$ S. Malvezzi, ${ }^{69 a}$ R. A. Manzoni, ${ }^{69 a, 69 b}$ D. Menasce, ${ }^{69 a}$ L. Moroni, ${ }^{69 a}$ M. Paganoni, ${ }^{69 a, 69 b}$ K. Pauwels, ${ }^{69 a, 69 b}$ D. Pedrini, ${ }^{69 a}$ S. Pigazzini, ${ }^{69 a, 69 b, e e}$ S. Ragazzi, ${ }^{69 a, 69 b}$ T. Tabarelli de Fatis, ${ }^{69 a, 69 b}$ S. Buontempo, ${ }^{70 a}$ N. Cavallo, ${ }^{70 a, 70 c}$ S. Di Guida, ${ }^{70 a, 70 d, p}$ F. Fabozzi, ${ }^{70 a, 70 c}$ F. Fienga, ${ }^{70 a, 70 b}$ A. O. M. Iorio, ${ }^{70 a, 70 b}$ W. A. Khan, ${ }^{70 a}$ L. Lista, ${ }^{70 a}$ S. Meola, ${ }^{70 a, 70 d, p}$ P. Paolucci, ${ }^{70 a, p}$ C. Sciacca, ${ }^{70 a, 70 b}$ F. Thyssen, ${ }^{70 a}$ P. Azzi, ${ }^{71 a}$ N. Bacchetta, ${ }^{71 a}$ L. Benato, $^{71 a, 71 b}$ D. Bisello, ${ }^{71 a, 71 b}$ A. Boletti, ${ }^{71 a, 71 b}$ R. Carlin, ${ }^{71 a, 71 b}$ P. Checchia, ${ }^{71 a}$ M. Dall'Osso, ${ }^{71 a, 71 b}$ P. De Castro Manzano, ${ }^{71 a}$ T. Dorigo, ${ }^{71 a}$ U. Dosselli, ${ }^{71 a}$ F. Gasparini, ${ }^{71 a, 71 b}$ U. Gasparini, ${ }^{71 a, 71 b}$ A. Gozzelino, ${ }^{71 a}$ S. Lacaprara, ${ }^{71 a}$ P. Lujan, ${ }^{71 a}$ M. Margoni, ${ }^{71 a, 71 b}$ A. T. Meneguzzo, ${ }^{71 a, 71 b}$ N. Pozzobon, ${ }^{71 a, 71 b}$ P. Ronchese, ${ }^{71 a, 71 b}$ R. Rossin, ${ }^{71 a, 71 b}$ E. Torassa, ${ }^{71 a}$ S. Ventura, ${ }^{71 a}$ M. Zanetti, ${ }^{71 a, 71 b}$ P. Zotto, ${ }^{71 a, 71 b}$ G. Zumerle, ${ }^{71 a, 71 b}$ A. Braghieri, ${ }^{72 a}$ A. Magnani, ${ }^{72 a}$ P. Montagna, ${ }^{72 a, 72 b}$ S. P. Ratti, ${ }^{72 a, 72 b}$ V. Re, ${ }^{72 a}$ M. Ressegotti, ${ }^{72 a, 72 b}$ C. Riccardi, ${ }^{72 a, 72 b}$ P. Salvini, ${ }^{72 a}$ I. Vai, ${ }^{72 a, 72 b}$ P. Vitulo, ${ }^{72 a, 72 b}$ L. Alunni Solestizi, ${ }^{73 a, 73 b}$ M. Biasini, ${ }^{73 a, 73 b}$ G. M. Bilei, ${ }^{73 a}$ C. Cecchi, ${ }^{73 a, 73 b}$ D. Ciangottini, ${ }^{73 a, 73 b}$ L. Fanò, ${ }^{73 a, 73 b}$ P. Lariccia, ${ }^{73 a, 73 b}$ R. Leonardi, ${ }^{73 a, 73 b}$ E. Manoni, ${ }^{73 a}$ G. Mantovani, ${ }^{73 a, 73 b}$ V. Mariani, ${ }^{73 a, 73 b}$ M. Menichelli, ${ }^{73 a}$ A. Rossi, ${ }^{73 a, 73 b}$ A. Santocchia, ${ }^{73 a, 73 b}$ D. Spiga, ${ }^{73 a}$ K. Androsov, ${ }^{74 a}$ P. Azzurri, ${ }^{74 a, p}$ G. Bagliesi, ${ }^{74 a}$ T. Boccali, ${ }^{74 a}$ L. Borrello, ${ }^{74 a}$ R. Castaldi, ${ }^{74 a}$ M. A. Ciocci, ${ }^{74 a, 74 b}$ R. Dell' Orso, ${ }^{74 a}$ G. Fedi, ${ }^{74 a}$ L. Giannini, ${ }^{74 a, 74 c}$ A. Giassi, ${ }^{74 a}$ M. T. Grippo, ${ }^{74 a, d d}$ F. Ligabue, ${ }^{74 a, 74 c}$ T. Lomtadze, ${ }^{74 a}$ E. Manca, ${ }^{74 a, 74 c}$ G. Mandorli, ${ }^{74 a, 74 c}$ A. Messineo, ${ }^{74 a, 74 b}$ F. Palla, ${ }^{74 a}$ A. Rizzi, ${ }^{74 a, 74 b}$ A. Savoy-Navarro, ${ }^{74 a, f f}$ P. Spagnolo, ${ }^{74 a}$ R. Tenchini, ${ }^{74 a}$ G. Tonelli, ${ }^{74 a, 74 b}$

A. Venturi, ${ }^{74 a}$ P. G. Verdini, ${ }^{74 a}$ L. Barone, ${ }^{75 a, 75 b}$ F. Cavallari, ${ }^{75 a}$ M. Cipriani, ${ }^{75 a, 75 b}$ N. Daci, ${ }^{75 a}$ D. Del Re, ${ }^{75 a, 75 b, p}$ E. Di Marco, ${ }^{75 a, 75 b}$ M. Diemoz, ${ }^{75 a}$ S. Gelli, ${ }^{75 a, 75 b}$ E. Longo, ${ }^{75 a, 75 b}$ F. Margaroli, ${ }^{75 a, 75 b}$ B. Marzocchi, ${ }^{75 a, 75 b}$ P. Meridiani, ${ }^{75 a}$ G. Organtini, ${ }^{75 a, 75 b}$ R. Paramatti, ${ }^{75 a, 75 b}$ F. Preiato, ${ }^{75 a, 75 b}$ S. Rahatlou, ${ }^{75 a, 75 b}$ C. Rovelli, ${ }^{75 a}$ F. Santanastasio, ${ }^{75 a, 75 b}$ N. Amapane, ${ }^{76 a, 76 b}$ R. Arcidiacono, ${ }^{76 a, 76 c}$ S. Argiro, ${ }^{76 a, 76 b}$ M. Arneodo, ${ }^{76 a, 76 c}$ N. Bartosik, ${ }^{76 a}$ R. Bellan, ${ }^{76 a, 76 b}$ C. Biino, ${ }^{76 a}$ N. Cartiglia, ${ }^{76 a}$ F. Cenna, ${ }^{76 a, 76 b}$ M. Costa, ${ }^{76 a, 76 b}$ R. Covarelli, ${ }^{76 a, 76 b}$ A. Degano, ${ }^{76 a, 76 b}$ N. Demaria, ${ }^{76 a}$ B. Kiani, ${ }^{76 a, 76 b}$ C. Mariotti, ${ }^{76 a}$ S. Maselli, ${ }^{76 a}$ E. Migliore, ${ }^{76 a, 76 b}$ V. Monaco, ${ }^{76 a, 76 b}$ E. Monteil, ${ }^{76 a, 76 b}$ M. Monteno, ${ }^{76 a}$ M. M. Obertino, ${ }^{76 a, 76 b}$ L. Pacher, ${ }^{76 a, 76 b}$ N. Pastrone, ${ }^{76 a}$ M. Pelliccioni, ${ }^{76 a}$ G. L. Pinna Angioni, ${ }^{76 a, 76 b}$ A. Romero, ${ }^{76 a, 76 b}$ M. Ruspa, ${ }^{76 a, 76 c}$ R. Sacchi, ${ }^{76,76 b}$ K. Shchelina, ${ }^{76,76 b}$ V. Sola, ${ }^{76 a}$ A. Solano, ${ }^{76,76 b}$ A. Staiano, ${ }^{76 a}$ P. Traczyk, ${ }^{76,76 b}$ S. Belforte, ${ }^{77 a}$ M. Casarsa, ${ }^{77}$ F. Cossutti, ${ }^{77 a}$ G. Della Ricca, ${ }^{77 a, 77 b}$ A. Zanetti, ${ }^{77 a}$ D. H. Kim, ${ }^{78}$ G. N. Kim, ${ }^{78}$ M. S. Kim, ${ }^{78}$ J. Lee, $^{78}$ S. Lee, ${ }^{78}$ S. W. Lee, ${ }^{78}$ C. S. Moon, ${ }^{78}$ Y. D. Oh, ${ }^{78}$ S. Sekmen, ${ }^{78}$ D. C. Son, ${ }^{78}$ Y. C. Yang, ${ }^{78}$ A. Lee, ${ }^{79}$ H. Kim, ${ }^{80}$ D. H. Moon, ${ }^{80}$ G. Oh, ${ }^{80}$ J. A. Brochero Cifuentes, ${ }^{81}$ J. Goh, ${ }^{81}$ T. J. Kim, ${ }^{81}$ S. Cho, ${ }^{82}$ S. Choi, ${ }^{82}$ Y. Go, ${ }^{82}$ D. Gyun, ${ }^{82}$ S. Ha, ${ }^{82}$ B. Hong, ${ }^{82}$ Y. Jo, ${ }^{82}$ Y. Kim, ${ }^{82}$ K. Lee, ${ }^{82}$ K. S. Lee, ${ }^{82}$ S. Lee, ${ }^{82}$ J. Lim, ${ }^{82}$ S. K. Park, ${ }^{82}$ Y. Roh, ${ }^{82}$ J. Almond, ${ }^{83}$ J. Kim, $^{83}$ J. S. Kim, ${ }^{83}$ H. Lee, ${ }^{83}$ K. Lee, ${ }^{83}$ K. Nam, ${ }^{83}$ S. B. Oh, ${ }^{83}$ B. C. Radburn-Smith, ${ }^{83}$ S. h. Seo, ${ }^{83}$ U. K. Yang, ${ }^{83}$ H. D. Yoo, ${ }^{83}$ G. B. Yu, ${ }^{83}$ H. Kim, ${ }^{84}$ J. H. Kim, ${ }^{84}$ J. S. H. Lee, ${ }^{84}$ I. C. Park, ${ }^{84}$ Y. Choi, ${ }^{85}$ C. Hwang, ${ }^{85}$ J. Lee, ${ }^{85}$ I. Yu, ${ }^{85}$ V. Dudenas, ${ }^{86}$ A. Juodagalvis, ${ }^{86}$ J. Vaitkus, ${ }^{86}$ I. Ahmed, ${ }^{87}$ Z. A. Ibrahim, ${ }^{87}$ M. A. B. Md Ali, ${ }^{87, g g}$ F. Mohamad Idris, ${ }^{87, \text { hh }}$ W. A. T. Wan Abdullah, ${ }^{87}$ M. N. Yusli, ${ }^{87}$ Z. Zolkapli, ${ }^{87}$ R Reyes-Almanza, ${ }^{88}$ G. Ramirez-Sanchez, ${ }^{88}$ M. C. Duran-Osuna, ${ }^{88}$ H. Castilla-Valdez, ${ }^{88}$

E. De La Cruz-Burelo, ${ }^{88}$ I. Heredia-De La Cruz, ${ }^{88, i i}$ R. I. Rabadan-Trejo, ${ }^{88}$ R. Lopez-Fernandez, ${ }^{88}$ J. Mejia Guisao, ${ }^{88}$ A. Sanchez-Hernandez, ${ }^{88}$ S. Carrillo Moreno, ${ }^{89}$ C. Oropeza Barrera, ${ }^{89}$ F. Vazquez Valencia, ${ }^{89}$ J. Eysermans, ${ }^{90}$ I. Pedraza, ${ }^{90}$ H. A. Salazar Ibarguen, ${ }^{90}$ C. Uribe Estrada, ${ }^{90}$ A. Morelos Pineda, ${ }^{91}$ D. Krofcheck, ${ }^{92}$ P. H. Butler, ${ }^{93}$ A. Ahmad, ${ }^{94}$ M. Ahmad, ${ }^{94}$ Q. Hassan, ${ }^{94}$ H. R. Hoorani, ${ }^{94}$ A. Saddique, ${ }^{94}$ M. A. Shah, ${ }^{94}$ M. Shoaib, ${ }^{94}$ M. Waqas, ${ }^{94}$ H. Bialkowska, ${ }^{95}$ M. Bluj, ${ }^{95}$ B. Boimska, ${ }^{95}$ T. Frueboes, ${ }^{95}$ M. Górski, ${ }^{95}$ M. Kazana, ${ }^{95}$ K. Nawrocki, ${ }^{95}$ M. Szleper, ${ }^{95}$ P. Zalewski, ${ }^{95}$ K. Bunkowski, ${ }^{96}$ A. Byszuk, ${ }^{96, j j}$ K. Doroba, ${ }^{96}$ A. Kalinowski, ${ }^{96}$ M. Konecki, ${ }^{96}$ J. Krolikowski, $^{96}$ M. Misiura, ${ }^{96}$ M. Olszewski, ${ }^{96}$ A. Pyskir, ${ }^{96}$ M. Walczak, ${ }^{96}$ P. Bargassa, ${ }^{97}$ C. Beirão Da Cruz E Silva, ${ }^{97}$ A. Di Francesco, ${ }^{97}$ P. Faccioli, ${ }^{97}$ B. Galinhas, ${ }^{97}$ M. Gallinaro, ${ }^{97}$ J. Hollar, ${ }^{97}$ N. Leonardo, ${ }^{97}$ L. Lloret Iglesias, ${ }^{97}$ M. V. Nemallapudi, ${ }^{97}$ J. Seixas, ${ }^{97}$ G. Strong, ${ }^{97}$ O. Toldaiev, ${ }^{97}$ D. Vadruccio, ${ }^{97}$ J. Varela, ${ }^{97}$ S. Afanasiev, ${ }^{98}$ V. Alexakhin, ${ }^{98}$ M. Gavrilenko, ${ }^{98}$ A. Golunov, ${ }^{98}$ I. Golutvin, ${ }^{98}$ N. Gorbounov, ${ }^{98}$ V. Karjavin, ${ }^{98}$ A. Lanev, ${ }^{98}$ A. Malakhov, ${ }^{98}$ V. Matveev, ${ }^{98, k k, 11}$ P. Moisenz, ${ }^{98}$ V. Palichik, ${ }^{98}$ V. Perelygin, ${ }^{98}$ M. Savina, ${ }^{98}$ S. Shmatov, ${ }^{98}$ N. Skatchkov, ${ }^{98}$ V. Smirnov, ${ }^{98}$ N. Voytishin, ${ }^{98}$ A. Zarubin, ${ }^{98}$ Y. Ivanov, ${ }^{99}$ V. Kim, ${ }^{99, m m}$ E. Kuznetsova, ${ }^{99, n n}$ P. Levchenko, ${ }^{99}$ V. Murzin, ${ }^{99}$ V. Oreshkin, ${ }^{99}$ I. Smirnov, ${ }^{99}$ D. Sosnov, ${ }^{99}$ V. Sulimov, ${ }^{99}$ L. Uvarov, ${ }^{99}$ S. Vavilov, ${ }^{99}$ A. Vorobyev, ${ }^{99}$ Yu. Andreev, ${ }^{100}$ A. Dermenev, ${ }^{100}$ S. Gninenko, ${ }^{100}$ N. Golubev, ${ }^{100}$ A. Karneyeu, ${ }^{100}$ M. Kirsanov, ${ }^{100}$ N. Krasnikov, ${ }^{100}$ A. Pashenkov, ${ }^{100}$ D. Tlisov, ${ }^{100}$ A. Toropin, ${ }^{100}$ V. Epshteyn, ${ }^{101}$ V. Gavrilov, ${ }^{101}$ N. Lychkovskaya, ${ }^{101}$ V. Popov, ${ }^{101}$ I. Pozdnyakov, ${ }^{101}$ G. Safronov, ${ }^{101}$ A. Spiridonov, ${ }^{101}$ A. Stepennov, ${ }^{101}$ V. Stolin, ${ }^{101}$ M. Toms, ${ }^{101}$ E. Vlasov, ${ }^{101}$ A. Zhokin, ${ }^{101}$ T. Aushev, ${ }^{102}$ A. Bylinkin, ${ }^{102,11}$ M. Chadeeva, ${ }^{103,00}$ P. Parygin, ${ }^{103}$ D. Philippov, ${ }^{103}$ S. Polikarpov, ${ }^{103}$ E. Popova, ${ }^{103}$ V. Rusinov, ${ }^{103}$ V. Andreev, ${ }^{104}$ M. Azarkin, ${ }^{104,11}$ I. Dremin, ${ }^{104,11}$ M. Kirakosyan, ${ }^{104,11}$ 
S. V. Rusakov, ${ }^{104}$ A. Terkulov, ${ }^{104}$ A. Baskakov, ${ }^{105}$ A. Belyaev, ${ }^{105}$ E. Boos, ${ }^{105}$ M. Dubinin, ${ }^{105, p p}$ L. Dudko, ${ }^{105}$ A. Ershov, ${ }^{105}$ A. Gribushin, ${ }^{105}$ V. Klyukhin, ${ }^{105}$ O. Kodolova, ${ }^{105}$ I. Lokhtin, ${ }^{105}$ I. Miagkov, ${ }^{105}$ S. Obraztsov, ${ }^{105}$ S. Petrushanko, ${ }^{105}$ V. Savrin, ${ }^{105}$ A. Snigirev, ${ }^{105}$ V. Blinov, ${ }^{106, q q}$ D. Shtol, ${ }^{106, q q}$ Y. Skovpen, ${ }^{106, q q}$ I. Azhgirey, ${ }^{107}$ I. Bayshev, ${ }^{107}$ S. Bitioukov, ${ }^{107}$ D. Elumakhov, ${ }^{107}$ A. Godizov, ${ }^{107}$ V. Kachanov, ${ }^{107}$ A. Kalinin, ${ }^{107}$ D. Konstantinov, ${ }^{107}$ P. Mandrik, ${ }^{107}$ V. Petrov, ${ }^{107}$ R. Ryutin, ${ }^{107}$ A. Sobol, ${ }^{107}$ S. Troshin, ${ }^{107}$ N. Tyurin, ${ }^{107}$ A. Uzunian, ${ }^{107}$ A. Volkov, ${ }^{107}$ P. Adzic, ${ }^{108, r r}$ P. Cirkovic, ${ }^{108}$ D. Devetak, ${ }^{108}$ M. Dordevic, ${ }^{108}$ J. Milosevic, ${ }^{108}$ V. Rekovic, ${ }^{108}$ J. Alcaraz Maestre, ${ }^{109}$ I. Bachiller, ${ }^{109}$ M. Barrio Luna, ${ }^{109}$ M. Cerrada, ${ }^{109}$ N. Colino, ${ }^{109}$ B. De La Cruz, ${ }^{109}$ A. Delgado Peris, ${ }^{109}$ C. Fernandez Bedoya, ${ }^{109}$ J. P. Fernández Ramos, ${ }^{109}$ J. Flix, ${ }^{109}$ M. C. Fouz, ${ }^{109}$ O. Gonzalez Lopez, ${ }^{109}$ S. Goy Lopez, ${ }^{109}$ J. M. Hernandez, ${ }^{109}$ M. I. Josa, ${ }^{109}$ D. Moran, ${ }^{109}$ A. Pérez-Calero Yzquierdo, ${ }^{109}$ J. Puerta Pelayo, ${ }^{109}$ I. Redondo, ${ }^{109}$ L. Romero, ${ }^{109}$ M. S. Soares, ${ }^{109}$ A. Álvarez Fernández, ${ }^{109}$ C. Albajar, ${ }^{10}$ J. F. de Trocóniz, ${ }^{110}$ M. Missiroli, ${ }^{110}$ J. Cuevas, ${ }^{111}$ C. Erice,${ }^{111}$ J. Fernandez Menendez, ${ }^{111}$

I. Gonzalez Caballero, ${ }^{111}$ J. R. González Fernández, ${ }^{111}$ E. Palencia Cortezon, ${ }^{111}$ S. Sanchez Cruz, ${ }^{111}$ P. Vischia, ${ }^{111}$ J. M. Vizan Garcia, ${ }^{111}$ I. J. Cabrillo, ${ }^{112}$ A. Calderon, ${ }^{112}$ B. Chazin Quero, ${ }^{112}$ E. Curras, ${ }^{112}$ J. Duarte Campderros, ${ }^{112}$ M. Fernandez, ${ }^{112}$ J. Garcia-Ferrero, ${ }^{112}$ G. Gomez, ${ }^{112}$ A. Lopez Virto, ${ }^{112}$ J. Marco, ${ }^{112}$ C. Martinez Rivero, ${ }^{112}$ P. Martinez Ruiz del Arbol, ${ }^{112}$ F. Matorras, ${ }^{112}$ J. Piedra Gomez, ${ }^{112}$ T. Rodrigo, ${ }^{12}$ A. Ruiz-Jimeno, ${ }^{112}$ L. Scodellaro, ${ }^{112}$ N. Trevisani, ${ }^{112}$ I. Vila, ${ }^{112}$ R. Vilar Cortabitarte, ${ }^{112}$ D. Abbaneo, ${ }^{113}$ B. Akgun, ${ }^{113}$ E. Auffray, ${ }^{113}$ P. Baillon, ${ }^{113}$ A. H. Ball, ${ }^{113}$ D. Barney, ${ }^{113}$ J. Bendavid, ${ }^{113}$ M. Bianco, ${ }^{113}$ P. Bloch, ${ }^{113}$ A. Bocci, ${ }^{113}$ C. Botta, ${ }^{113}$ T. Camporesi, ${ }^{113}$ R. Castello, ${ }^{113}$ M. Cepeda, ${ }^{113}$ G. Cerminara, ${ }^{113}$ E. Chapon, ${ }^{113}$ Y. Chen, ${ }^{113}$ D. d'Enterria, ${ }^{113}$ A. Dabrowski, ${ }^{113}$ V. Daponte, ${ }^{113}$ A. David, ${ }^{113}$ M. De Gruttola, ${ }^{113}$ A. De Roeck, ${ }^{113}$ N. Deelen, ${ }^{113}$ M. Dobson, ${ }^{113}$ T. du Pree, ${ }^{113}$ M. Dünser ${ }^{113}$ N. Dupont, ${ }^{113}$ A. Elliott-Peisert, ${ }^{113}$ P. Everaerts, ${ }^{113}$ F. Fallavollita, ${ }^{113}$ G. Franzoni, ${ }^{113}$ J. Fulcher, ${ }^{113}$ W. Funk, ${ }^{113}$ D. Gigi, ${ }^{113}$ A. Gilbert, ${ }^{113}$ K. Gill, ${ }^{13}$ F. Glege, ${ }^{113}$ D. Gulhan, ${ }^{113}$ P. Harris, ${ }^{113}$ J. Hegeman, ${ }^{113}$ V. Innocente, ${ }^{113}$ A. Jafari, ${ }^{113}$ P. Janot, ${ }^{113}$

O. Karacheban, ${ }^{11, s}$ J. Kieseler, ${ }^{113}$ V. Knünz, ${ }^{113}$ A. Kornmayer, ${ }^{113}$ M. J. Kortelainen, ${ }^{113}$ M. Krammer, ${ }^{113, b}$ C. Lange, ${ }^{113}$ P. Lecoq, ${ }^{113}$ C. Lourenço, ${ }^{113}$ M. T. Lucchini, ${ }^{113}$ L. Malgeri, ${ }^{113}$ M. Mannelli, ${ }^{113}$ A. Martelli, ${ }^{113}$ F. Meijers, ${ }^{113}$ J. A. Merlin, ${ }^{113}$ S. Mersi, ${ }^{113}$ E. Meschi, ${ }^{113}$ P. Milenovic, ${ }^{113, s s}$ F. Moortgat, ${ }^{113}$ M. Mulders, ${ }^{113}$ H. Neugebauer, ${ }^{113}$ J. Ngadiuba, ${ }^{113}$ S. Orfanelli, ${ }_{113}^{13}$ L. Orsini, ${ }^{113}$ L. Pape, ${ }^{113}$ E. Perez,${ }^{113}$ M. Peruzzi,${ }^{113}$ A. Petrilli, ${ }^{113}$ G. Petrucciani, ${ }^{113}$ A. Pfeiffer, ${ }^{113}$ M. Pierini, ${ }^{113}$ D. Rabady, ${ }^{113}$ A. Racz, ${ }^{113}$ T. Reis, ${ }^{113}$ G. Rolandi, ${ }^{113, t t}$ M. Rovere, ${ }^{113}$ H. Sakulin,${ }^{113}$ C. Schäfer, ${ }^{113}$ C. Schwick, ${ }^{113}$ M. Seidel, ${ }^{113}$ M. Selvaggi, ${ }^{113}$ A. Sharma, ${ }^{113}$ P. Silva, ${ }^{113}$ P. Sphicas, ${ }^{113, \text { uu }}$ A. Stakia, ${ }^{113}$ J. Steggemann, ${ }^{113}$ M. Stoye,${ }^{113}$ M. Tosi,${ }^{113}$ D. Treille, ${ }^{113}$ A. Triossi, ${ }^{113}$ A. Tsirou, ${ }^{113}$ V. Veckalns, ${ }^{113, v v}$ M. Verweij,${ }^{113}$ W. D. Zeuner,${ }^{113}$ W. Bertl, ${ }^{114, a}$ L. Caminada, ${ }^{14, w w}$ K. Deiters, ${ }^{114}$ W. Erdmann, ${ }^{114}$ R. Horisberger, ${ }^{114}$ Q. Ingram, ${ }^{114}$ H. C. Kaestli, ${ }^{114}$ D. Kotlinski, ${ }^{114}$ U. Langenegger, ${ }^{114}$ T. Rohe, ${ }^{114}$ S. A. Wiederkehr, ${ }^{114}$ M. Backhaus, ${ }^{115}$ L. Bäni, ${ }^{115}$ P. Berger,${ }^{115}$ L. Bianchini, ${ }^{115}$ B. Casal,${ }^{115}$ G. Dissertori, ${ }^{115}$ M. Dittmar, ${ }^{115}$ M. Donegà, ${ }^{115}$ C. Dorfer, ${ }^{115}$ C. Grab, ${ }^{115}$ C. Heidegger, ${ }^{115}$ D. Hits, ${ }^{115}$ J. Hoss,${ }^{115}$ G. Kasieczka, ${ }^{115}$ T. Klijnsma, ${ }^{115}$ W. Lustermann, ${ }^{115}$ B. Mangano, ${ }^{115}$ M. Marionneau, ${ }^{115}$ M. T. Meinhard, ${ }^{115}$ D. Meister, ${ }^{115}$ F. Micheli, ${ }^{115}$ P. Musella, ${ }^{115}$ F. Nessi-Tedaldi, ${ }^{115}$ F. Pandolfi, ${ }^{115}$ J. Pata,${ }^{115}$ F. Pauss, ${ }^{115}$ G. Perrin, ${ }^{115}$ L. Perrozzi, ${ }^{115}$ M. Quittnat, ${ }^{115}$ M. Reichmann, ${ }^{115}$ D. A. Sanz Becerra, ${ }^{115}$ M. Schönenberger, ${ }^{115}$ L. Shchutska, ${ }^{115}$ V. R. Tavolaro, ${ }^{115}$ K. Theofilatos, ${ }^{115}$ M. L. Vesterbacka Olsson, ${ }^{115}$ R. Wallny, ${ }^{115}$ D. H. Zhu, ${ }^{15}$ T. K. Aarrestad, ${ }^{116}$ C. Amsler, ${ }^{116, x x}$ M. F. Canelli, ${ }^{116}$ A. De Cosa, ${ }^{116}$ R. Del Burgo, ${ }^{116}$ S. Donato, ${ }^{116}$ C. Galloni, ${ }^{116}$ T. Hreus, ${ }^{116}$ B. Kilminster, ${ }^{116}$ D. Pinna, ${ }^{116}$ G. Rauco, ${ }^{116}$ P. Robmann, ${ }^{116}$ D. Salerno, ${ }^{116}$ K. Schweiger, ${ }^{116}$ C. Seitz, ${ }^{116}$ Y. Takahashi, ${ }^{116}$ A. Zucchetta, ${ }^{116}$ V. Candelise, ${ }^{117}$ Y. H. Chang, ${ }^{117}$ K. y. Cheng, ${ }^{117}$ T. H. Doan, ${ }^{117}$ Sh. Jain, ${ }^{117}$ R. Khurana, ${ }^{117}$ C. M. Kuo, ${ }^{117}$ W. Lin,${ }^{117}$

A. Pozdnyakov, ${ }^{117}$ S. S. Yu, ${ }^{117}$ Arun Kumar, ${ }^{118}$ P. Chang, ${ }^{118}$ Y. Chao,${ }^{118}$ K. F. Chen, ${ }^{118}$ P. H. Chen, ${ }^{118}$ F. Fiori, ${ }^{118}$ W.-S. Hou, ${ }^{118}$ Y. Hsiung, ${ }^{118}$ Y. F. Liu, ${ }^{118}$ R.-S. Lu, ${ }^{118}$ E. Paganis, ${ }^{118}$ A. Psallidas, ${ }^{118}$ A. Steen, ${ }^{118}$ J. f. Tsai, ${ }^{118}$ B. Asavapibhop, ${ }^{119}$ K. Kovitanggoon, ${ }^{119}$ G. Singh, ${ }^{119}$ N. Srimanobhas, ${ }^{119}$ A. Bat, ${ }^{120}$ F. Boran, ${ }^{120}$ S. Cerci, ${ }^{120, y y}$ S. Damarseckin, ${ }^{120}$ Z. S. Demiroglu, ${ }^{120}$ C. Dozen, ${ }^{120}$ I. Dumanoglu, ${ }^{120}$ S. Girgis, ${ }^{120}$ G. Gokbulut, ${ }^{120}$ Y. Guler, ${ }^{120}$ I. Hos, ${ }^{120, z z}$ E. E. Kangal, ${ }^{120 \text {,aaa }}$ O. Kara, ${ }^{120}$ A. Kayis Topaksu, ${ }^{120}$ U. Kiminsu, ${ }^{120}$ M. Oglakci, ${ }^{120}$ G. Onengut, ${ }^{120, b b b}$ K. Ozdemir, ${ }^{120, \text { ccc }}$ D. Sunar Cerci, ${ }^{120, y y}$ B. Tali, ${ }^{120, y y}$ U. G. Tok, ${ }^{120}$ S. Turkcapar, ${ }^{120}$ I. S. Zorbakir, ${ }^{120}$ C. Zorbilmez, ${ }^{120}$ G. Karapinar, ${ }^{121, \text { ddd }}$ K. Ocalan, ${ }^{121, \text { eee }}$ M. Yalvac, ${ }^{121}$ M. Zeyrek, ${ }^{121}$ E. Gülmez,${ }^{122}$ M. Kaya, ${ }^{122, f f f}$ O. Kaya, ${ }^{122, \text { ggg }}$ S. Tekten, ${ }^{122}$ E. A. Yetkin, ${ }^{122, \text { hhh }}$ M. N. Agaras, ${ }^{123}$ S. Atay, ${ }^{123}$ A. Cakir, ${ }^{123}$ K. Cankocak, ${ }^{123}$ Y. Komurcu, ${ }^{123}$ B. Grynyov, ${ }^{124}$ L. Levchuk, ${ }^{125}$ F. Ball, ${ }^{126}$ L. Beck, ${ }^{126}$ J. J. Brooke, ${ }^{126}$ D. Burns, ${ }^{126}$ E. Clement, ${ }^{126}$ D. Cussans, ${ }^{126}$ O. Davignon, ${ }^{126}$ H. Flacher, ${ }^{126}$ J. Goldstein, ${ }^{126}$ G. P. Heath, ${ }^{126}$ H. F. Heath, ${ }^{126}$ L. Kreczko, ${ }^{126}$ D. M. Newbold, ${ }^{126, \text { iii }}$ S. Paramesvaran, ${ }^{126}$ T. Sakuma, ${ }^{126}$

S. Seif El Nasr-storey, ${ }^{126}$ D. Smith, ${ }^{126}$ V. J. Smith, ${ }^{126}$ K. W. Bell, ${ }^{127}$ A. Belyaev, ${ }^{127, j j j}$ C. Brew, ${ }^{127}$ R. M. Brown, ${ }^{127}$ L. Calligaris, ${ }^{127}$ D. Cieri, ${ }^{127}$ D. J. A. Cockerill, ${ }^{127}$ J. A. Coughlan, ${ }^{127}$ K. Harder, ${ }^{127}$ S. Harper, ${ }^{127}$ J. Linacre, ${ }^{127}$ E. Olaiya, ${ }^{127}$ 
D. Petyt, ${ }^{127}$ C. H. Shepherd-Themistocleous, ${ }^{127}$ A. Thea, ${ }^{127}$ I. R. Tomalin, ${ }^{127}$ T. Williams, ${ }^{127}$ W. J. Womersley, ${ }^{127}$ G. Auzinger, ${ }^{128}$ R. Bainbridge, ${ }^{128} \mathrm{~J}$. Borg, ${ }^{128}$ S. Breeze, ${ }^{128}$ O. Buchmuller, ${ }^{128}$ A. Bundock, ${ }^{128}$ S. Casasso, ${ }^{128}$ M. Citron, ${ }^{128}$ D. Colling, ${ }^{128}$ L. Corpe,${ }^{128}$ P. Dauncey, ${ }^{128}$ G. Davies, ${ }^{128}$ A. De Wit, ${ }^{128}$ M. Della Negra, ${ }^{128}$ R. Di Maria, ${ }^{128}$ A. Elwood, ${ }^{128}$ Y. Haddad, ${ }^{128}$ G. Hall, ${ }^{128}$ G. Iles, ${ }^{128}$ T. James, ${ }^{128}$ R. Lane, ${ }^{128}$ C. Laner, ${ }^{128}$ L. Lyons, ${ }^{128}$ A.-M. Magnan, ${ }^{128}$ S. Malik, ${ }^{128}$ L. Mastrolorenzo, ${ }^{128}$ T. Matsushita, ${ }^{128}$ J. Nash, ${ }^{128}$ A. Nikitenko, ${ }^{128, g}$ V. Palladino, ${ }^{128}$ M. Pesaresi, ${ }^{128}$ D. M. Raymond, ${ }^{128}$ A. Richards, ${ }^{128}$ A. Rose, ${ }^{128}$ E. Scott, ${ }^{128}$ C. Seez, ${ }^{128}$ A. Shtipliyski, ${ }^{128}$ S. Summers, ${ }^{128}$ A. Tapper, ${ }^{128}$ K. Uchida, ${ }^{128}$ M. Vazquez Acosta, ${ }^{128, k k k}$ T. Virdee, ${ }^{128, p}$ N. Wardle, ${ }^{128}$ D. Winterbottom, ${ }^{128}$ J. Wright, ${ }^{128}$ S. C. Zenz, ${ }^{128}$ J. E. Cole, ${ }^{129}$

P. R. Hobson, ${ }^{129}$ A. Khan, ${ }^{129}$ P. Kyberd,${ }^{129}$ I. D. Reid,${ }^{129}$ L. Teodorescu, ${ }^{129}$ S. Zahid, ${ }^{129}$ A. Borzou, ${ }^{130}$ K. Call, ${ }^{130}$ J. Dittmann, ${ }^{130}$ K. Hatakeyama, ${ }^{130}$ H. Liu, ${ }^{130}$ N. Pastika, ${ }^{130}$ C. Smith,${ }^{130}$ R. Bartek, ${ }^{131}$ A. Dominguez, ${ }^{131}$ A. Buccilli, ${ }^{132}$ S. I. Cooper, ${ }^{132}$ C. Henderson, ${ }^{132}$ P. Rumerio, ${ }^{132}$ C. West, ${ }^{132}$ D. Arcaro, ${ }^{133}$ A. Avetisyan, ${ }^{133}$ T. Bose, ${ }^{133}$ D. Gastler, ${ }^{133}$ D. Rankin, ${ }^{133}$ C. Richardson, ${ }^{133}$ J. Rohlf, ${ }^{133}$ L. Sulak, ${ }^{133}$ D. Zou, ${ }^{133}$ G. Benelli, ${ }^{134}$ D. Cutts, ${ }^{134}$ M. Hadley, ${ }^{134}$ J. Hakala, ${ }^{134}$ U. Heintz, ${ }^{134}$ J. M. Hogan, ${ }^{134}$ K. H. M. Kwok, ${ }^{134}$ E. Laird, ${ }^{134}$ G. Landsberg, ${ }^{134}$ J. Lee, ${ }^{134}$ Z. Mao, ${ }^{134}$ M. Narain, ${ }^{134}$ J. Pazzini, ${ }^{134}$ S. Piperov, ${ }^{134}$ S. Sagir, ${ }^{134}$ R. Syarif, ${ }^{134}$ D. Yu, ${ }^{134}$ R. Band,${ }^{135}$ C. Brainerd, ${ }^{135}$ R. Breedon, ${ }^{135}$ D. Burns, ${ }^{135}$ M. Calderon De La Barca Sanchez, ${ }^{135}$ M. Chertok, ${ }^{135}$ J. Conway, ${ }^{135}$ R. Conway, ${ }^{135}$ P. T. Cox, ${ }^{135}$ R. Erbacher, ${ }^{135}$ C. Flores, ${ }^{135}$ G. Funk,${ }^{135}$ W. Ko, ${ }^{135}$ R. Lander, ${ }^{135}$ C. Mclean, ${ }^{135}$ M. Mulhearn, ${ }^{135}$ D. Pellett, ${ }^{135}$ J. Pilot, ${ }^{135}$ S. Shalhout, ${ }^{135}$ M. Shi, ${ }^{135}$ J. Smith, ${ }^{135}$ D. Stolp, ${ }^{135}$ K. Tos, ${ }^{135}$ M. Tripathi, ${ }^{135}$ Z. Wang, ${ }^{135}$ M. Bachtis, ${ }^{136}$ C. Bravo, ${ }^{136}$ R. Cousins, ${ }^{136}$ A. Dasgupta, ${ }^{136}$ A. Florent, ${ }^{136}$ J. Hauser, ${ }^{136}$ M. Ignatenko, ${ }^{136}$ N. Mccoll, ${ }^{136}$ S. Regnard, ${ }^{136}$ D. Saltzberg, ${ }^{136}$ C. Schnaible, ${ }^{136}$ V. Valuev, ${ }^{136}$ E. Bouvier, ${ }^{137}$ K. Burt, ${ }^{137}$ R. Clare, ${ }^{137}$ J. Ellison, ${ }^{137}$ J. W. Gary, ${ }^{137}$ S. M. A. Ghiasi Shirazi, ${ }^{137}$ G. Hanson, ${ }^{137}$ J. Heilman, ${ }^{137}$ G. Karapostoli, ${ }^{137}$ E. Kennedy, ${ }^{137}$ F. Lacroix, ${ }^{137}$ O. R. Long, ${ }^{137}$ M. Olmedo Negrete, ${ }^{137}$ M. I. Paneva, ${ }^{137}$ W. Si, ${ }^{137}$ L. Wang, ${ }^{137}$ H. Wei, ${ }^{137}$ S. Wimpenny, ${ }^{137}$ B. R. Yates, ${ }^{137}$ J. G. Branson, ${ }^{138}$ S. Cittolin, ${ }^{138}$ M. Derdzinski, ${ }^{138}$ R. Gerosa, ${ }^{138}$ D. Gilbert, ${ }^{138}$ B. Hashemi, ${ }^{138}$ A. Holzner, ${ }^{138}$ D. Klein, ${ }^{138}$ G. Kole, ${ }^{138}$ V. Krutelyov, ${ }^{138}$ J. Letts, ${ }^{138}$ M. Masciovecchio, ${ }^{138}$ D. Olivito, ${ }^{138}$ S. Padhi, ${ }^{138}$ M. Pieri, ${ }^{138}$ M. Sani, ${ }^{138}$ V. Sharma, ${ }^{138}$ S. Simon, ${ }^{138}$ M. Tadel, ${ }^{138}$ A. Vartak, ${ }^{138}$ S. Wasserbaech,,${ }^{138,111}$ J. Wood, ${ }^{138}$ F. Würthwein, ${ }^{138}$ A. Yagil, ${ }^{138}$ G. Zevi Della Porta, ${ }^{138}$ N. Amin, ${ }^{139}$ R. Bhandari, ${ }^{139}$ J. Bradmiller-Feld, ${ }^{139}$ C. Campagnari, ${ }^{139}$ A. Dishaw, ${ }^{139}$ V. Dutta, ${ }^{139}$ M. Franco Sevilla, ${ }^{139}$ L. Gouskos, ${ }^{139}$ R. Heller, ${ }^{139}$ J. Incandela, ${ }^{139}$ A. Ovcharova, ${ }^{139}$ H. Qu, ${ }^{139}$ J. Richman, ${ }^{139}$ D. Stuart, ${ }^{139}$ I. Suarez, ${ }^{139}$ J. Yoo, ${ }^{139}$ D. Anderson, ${ }^{140}$ A. Bornheim, ${ }^{140}$ J. Bunn, ${ }^{140}$ J. M. Lawhorn, ${ }^{140}$ H. B. Newman, ${ }^{140}$ T. Q. Nguyen, ${ }^{140}$ C. Pena, ${ }^{140}$ M. Spiropulu, ${ }^{140}$ J. R. Vlimant, ${ }^{140}$ R. Wilkinson, ${ }^{140}$ S. Xie, ${ }^{140}$ Z. Zhang, ${ }^{140}$ R. Y. Zhu, ${ }^{140}$ M. B. Andrews, ${ }^{141}$ T. Ferguson, ${ }^{141}$ T. Mudholkar, ${ }^{141}$ M. Paulini, ${ }^{141}$ J. Russ, ${ }^{141}$ M. Sun, ${ }^{141}$ H. Vogel, ${ }^{141}$ I. Vorobiev, ${ }^{141}$ M. Weinberg, ${ }^{141}$ J. P. Cumalat, ${ }^{142}$ W. T. Ford, ${ }^{142}$ F. Jensen, ${ }^{142}$ A. Johnson, ${ }^{142}$ M. Krohn, ${ }^{142}$ S. Leontsinis, ${ }^{142}$ T. Mulholland, ${ }^{142}$ K. Stenson, ${ }^{142}$ S. R. Wagner, ${ }^{142}$ J. Alexander, ${ }^{143}$ J. Chaves, ${ }^{143}$ J. Chu, ${ }^{143}$ S. Dittmer, ${ }^{143}$ K. Mcdermott, ${ }^{143}$ N. Mirman, ${ }^{143}$ J. R. Patterson, ${ }^{143}$ D. Quach,${ }^{143}$ A. Rinkevicius, ${ }^{143}$ A. Ryd ${ }^{143}$ L. Skinnari, ${ }^{143}$ L. Soffi, ${ }^{143}$ S. M. Tan, ${ }^{143}$ Z. Tao, ${ }^{143}$ J. Thom, ${ }^{143}$ J. Tucker, ${ }^{143}$ P. Wittich, ${ }^{143}$ M. Zientek, ${ }^{143}$ S. Abdullin, ${ }^{144}$ M. Albrow, ${ }^{144}$ M. Alyari, ${ }^{144}$ G. Apollinari, ${ }^{144}$ A. Apresyan, ${ }^{144}$ A. Apyan, ${ }^{144}$ S. Banerjee,${ }^{144}$ L. A. T. Bauerdick, ${ }^{144}$ A. Beretvas, ${ }^{144}$ J. Berryhill, ${ }^{144}$ P. C. Bhat,${ }^{144}$ G. Bolla, ${ }^{144, a}$ K. Burkett, ${ }^{144}$ J. N. Butler, ${ }^{144}$ A. Canepa, ${ }^{144}$ G. B. Cerati, ${ }^{144}$ H. W. K. Cheung, ${ }^{144}$ F. Chlebana, ${ }^{144}$ M. Cremonesi, ${ }^{144}$ J. Duarte, ${ }^{144}$ V. D. Elvira, ${ }^{144}$ J. Freeman, ${ }^{144}$ Z. Gecse,${ }^{144}$ E. Gottschalk, ${ }^{144}$ L. Gray, ${ }^{144}$ D. Green, ${ }^{144}$ S. Grünendahl, ${ }^{144}$ O. Gutsche, ${ }^{144}$ J. Hanlon, ${ }^{144}$ R. M. Harris,${ }^{144}$ S. Hasegawa, ${ }^{144}$ J. Hirschauer,${ }^{144}$ Z. Hu, ${ }^{144}$ B. Jayatilaka, ${ }^{144}$ S. Jindariani, ${ }^{144}$ M. Johnson, ${ }^{144}$ U. Joshi, ${ }^{144}$ B. Klima, ${ }^{144}$ B. Kreis, ${ }^{144}$ S. Lammel, ${ }^{144}$ D. Lincoln,,${ }^{144}$ R. Lipton, ${ }^{144}$ M. Liu, ${ }^{144}$ T. Liu, ${ }^{144}$ R. Lopes De Sá, ${ }^{144}$ J. Lykken, ${ }^{144}$ K. Maeshima,${ }^{144}$ N. Magini, ${ }^{144}$ J. M. Marraffino, ${ }^{144}$ D. Mason, ${ }^{144}$ P. McBride, ${ }^{144}$ P. Merkel, ${ }^{144}$ S. Mrenna, ${ }^{144}$ S. Nahn, ${ }^{144}$ V. O’Dell, ${ }^{144}$ K. Pedro, ${ }^{144}$ O. Prokofyev, ${ }^{144}$ G. Rakness, ${ }^{144}$ L. Ristori, ${ }^{144}$ B. Schneider, ${ }^{144}$ E. Sexton-Kennedy, ${ }^{144}$ A. Soha, ${ }^{144}$ W. J. Spalding, ${ }^{144}$ L. Spiegel, ${ }^{144}$ S. Stoynev, ${ }^{144}$ J. Strait, ${ }^{144}$ N. Strobbe, ${ }^{144}$ L. Taylor, ${ }^{144}$ S. Tkaczyk, ${ }^{144}$ N. V. Tran, ${ }^{144}$ L. Uplegger, ${ }^{144}$ E. W. Vaandering, ${ }^{144}$ C. Vernieri, ${ }^{144}$ M. Verzocchi, ${ }^{144}$ R. Vidal, ${ }^{144}$ M. Wang, ${ }^{144}$ H. A. Weber, ${ }^{144}$ A. Whitbeck, ${ }^{144}$ W. Wu, ${ }^{144}$ D. Acosta, ${ }^{145}$ P. Avery, ${ }^{145}$ P. Bortignon, ${ }^{145}$ D. Bourilkov, ${ }^{145}$ A. Brinkerhoff, ${ }^{145}$ A. Carnes, ${ }^{145}$ M. Carver, ${ }^{145}$ D. Curry, ${ }^{145}$ R. D. Field, ${ }^{145}$ I. K. Furic, ${ }^{145}$ S. V. Gleyzer, ${ }^{145}$ B. M. Joshi, ${ }^{145}$ J. Konigsberg, ${ }^{145}$ A. Korytov, ${ }^{145}$ K. Kotov, ${ }^{145}$ P. Ma, ${ }^{145}$ K. Matchev, ${ }^{145}$ H. Mei ${ }^{145}$ G. Mitselmakher, ${ }^{145}$ K. Shi, ${ }^{145}$ D. Sperka, ${ }^{145}$ N. Terentyev, ${ }^{145}$ L. Thomas, ${ }^{145}$ J. Wang, ${ }^{145}$ S. Wang, ${ }^{145}$ J. Yelton, ${ }^{145}$ Y. R. Joshi, ${ }^{146}$ S. Linn, ${ }^{146}$ P. Markowitz, ${ }^{146}$ J. L. Rodriguez,${ }^{146}$ A. Ackert, ${ }^{147}$ T. Adams, ${ }^{147}$ A. Askew, ${ }^{147}$ S. Hagopian, ${ }^{147}$ V. Hagopian, ${ }^{147}$ K. F. Johnson, ${ }^{147}$ T. Kolberg, ${ }^{147}$ G. Martinez, ${ }^{147}$ T. Perry, ${ }^{147}$ H. Prosper, ${ }^{147}$ A. Saha, ${ }^{147}$ A. Santra, ${ }^{147}$ V. Sharma, ${ }^{147}$ R. Yohay, ${ }^{147}$ M. M. Baarmand, ${ }^{148}$ V. Bhopatkar ${ }^{148}$ S. Colafranceschi, ${ }^{148}$ M. Hohlmann, ${ }^{148}$ D. Noonan, ${ }^{148}$ T. Roy, ${ }^{148}$ F. Yumiceva, ${ }^{148}$ M. R. Adams, ${ }^{149}$ L. Apanasevich, ${ }^{149}$ D. Berry, ${ }^{149}$ R. R. Betts, ${ }^{149}$ R. Cavanaugh,${ }^{149}$ X. Chen, ${ }^{149}$ O. Evdokimov, ${ }^{149}$ C. E. Gerber, ${ }^{149}$ D. A. Hangal, ${ }^{149}$ D. J. Hofman, ${ }^{149}$ K. Jung, ${ }^{149}$ J. Kamin, ${ }^{149}$ I. D. Sandoval Gonzalez, ${ }^{149}$ 
M. B. Tonjes, ${ }^{149}$ H. Trauger, ${ }^{149}$ N. Varelas, ${ }^{149}$ H. Wang, ${ }^{149}$ Z. Wu, ${ }^{149}$ J. Zhang, ${ }^{149}$ B. Bilki, ${ }^{150, m m m}$ W. Clarida, ${ }^{150}$ K. Dilsiz, ${ }^{150, \text { nnn }}$ S. Durgut, ${ }^{150}$ R. P. Gandrajula, ${ }^{150}$ M. Haytmyradov, ${ }^{150}$ V. Khristenko, ${ }^{150}$ J.-P. Merlo, ${ }^{150}$

H. Mermerkaya, ${ }^{150,000}$ A. Mestvirishvili, ${ }^{150}$ A. Moeller, ${ }^{150}$ J. Nachtman, ${ }^{150}$ H. Ogul, ${ }^{150, \text { ppp }}$ Y. Onel, ${ }^{150}$ F. Ozok, ${ }^{150, q q q}$ A. Penzo, ${ }^{150}$ C. Snyder, ${ }^{150}$ E. Tiras, ${ }^{150}$ J. Wetzel,${ }^{150}$ K. Yi,${ }^{150}$ B. Blumenfeld, ${ }^{151}$ A. Cocoros,${ }^{151}$ N. Eminizer, ${ }^{151}$ D. Fehling, ${ }^{151}$ L. Feng, ${ }^{151}$ A. V. Gritsan, ${ }^{151}$ P. Maksimovic, ${ }^{151}$ J. Roskes, ${ }^{151}$ U. Sarica, ${ }^{151}$ M. Swartz, ${ }^{151}$ M. Xiao, ${ }^{151}$ C. You, ${ }^{151}$ A. Al-bataineh, ${ }^{152}$ P. Baringer, ${ }^{152}$ A. Bean, ${ }^{152}$ S. Boren, ${ }^{152}$ J. Bowen, ${ }^{152}$ J. Castle, ${ }^{152}$ S. Khalil, ${ }^{152}$ A. Kropivnitskaya, ${ }^{152}$ D. Majumder, ${ }^{152}$ W. Mcbrayer, ${ }^{152}$ M. Murray, ${ }^{152}$ C. Rogan, ${ }^{152}$ C. Royon, ${ }^{152}$ S. Sanders, ${ }^{152}$ E. Schmitz, ${ }^{152}$ J. D. Tapia Takaki, ${ }^{152}$ Q. Wang, ${ }^{152}$ A. Ivanov, ${ }^{153}$ K. Kaadze ${ }^{153}$ Y. Maravin, ${ }^{153}$ A. Mohammadi, ${ }^{153}$ L. K. Saini, ${ }^{153}$ N. Skhirtladze, ${ }^{153}$ F. Rebassoo, ${ }^{154}$ D. Wright, ${ }^{154}$ A. Baden, ${ }^{155}$ O. Baron, ${ }^{155}$ A. Belloni, ${ }^{155}$ S. C. Eno, ${ }^{155}$ Y. Feng, ${ }^{155}$ C. Ferraioli, ${ }^{155}$ N. J. Hadley, ${ }^{155}$ S. Jabeen, ${ }^{155}$ G. Y. Jeng, ${ }^{155}$ R. G. Kellogg, ${ }^{155}$ J. Kunkle, ${ }^{155}$ A. C. Mignerey, ${ }^{155}$ F. Ricci-Tam, ${ }^{155}$ Y. H. Shin, ${ }^{155}$ A. Skuja, ${ }^{155}$ S. C. Tonwar, ${ }^{155}$ D. Abercrombie,${ }^{156}$ B. Allen, ${ }^{156}$ V. Azzolini, ${ }^{156}$ R. Barbieri, ${ }^{156}$ A. Baty, ${ }^{156}$ G. Bauer ${ }^{156}$ R. Bi ${ }^{156}$ S. Brandt, ${ }^{156}$ W. Busza, ${ }^{156}$ I. A. Cali, ${ }^{156}$ M. D’Alfonso, ${ }^{156}$ Z. Demiragli, ${ }^{156}$ G. Gomez Ceballos, ${ }^{156} \mathrm{M}$. Goncharov, ${ }^{156} \mathrm{D}$. Hsu, ${ }^{156} \mathrm{M}$. Hu,${ }^{156}$ Y. Iiyama, ${ }^{156} \mathrm{G}$. M. Innocenti, ${ }^{156} \mathrm{M}$. Klute, ${ }^{156}$ D. Kovalskyi, ${ }^{156}$ Y.-J. Lee, ${ }^{156}$ A. Levin, ${ }^{156}$ P. D. Luckey, ${ }^{156}$ B. Maier, ${ }^{156}$ A. C. Marini, ${ }^{156}$ C. Mcginn, ${ }^{156}$ C. Mironov, ${ }^{156}$ S. Narayanan, ${ }^{156}$ X. Niu, ${ }^{156}$ C. Paus, ${ }^{156}$ C. Roland,${ }^{156}$ G. Roland, ${ }^{156}$ J. Salfeld-Nebgen, ${ }^{156}$ G. S. F. Stephans, ${ }^{156}$ K. Sumorok, ${ }^{156}$ K. Tatar, ${ }^{156}$ D. Velicanu, ${ }^{156}$ J. Wang, ${ }^{156}$ T. W. Wang, ${ }^{156}$ B. Wyslouch, ${ }^{156}$ A. C. Benvenuti, ${ }^{157}$ R. M. Chatterjee, ${ }^{157}$ A. Evans, ${ }^{157}$ P. Hansen, ${ }^{157}$ J. Hiltbrand, ${ }^{157}$ S. Kalafut, ${ }^{157}$ Y. Kubota, ${ }^{157}$ Z. Lesko, ${ }^{157}$ J. Mans, ${ }^{157}$ S. Nourbakhsh, ${ }^{157}$ N. Ruckstuhl, ${ }^{157}$ R. Rusack, ${ }^{157}$ J. Turkewitz, ${ }^{157}$ M. A. Wadud, ${ }^{157}$ J. G. Acosta, ${ }^{158}$ S. Oliveros, ${ }^{158}$ E. Avdeeva, ${ }^{159}$ K. Bloom, ${ }^{159}$ D. R. Claes, ${ }^{159}$ C. Fangmeier, ${ }^{159}$ F. Golf, ${ }^{159}$ R. Gonzalez Suarez, ${ }^{159}$ R. Kamalieddin, ${ }^{159}$ I. Kravchenko, ${ }^{159}$ J. Monroy, ${ }^{159}$ J. E. Siado, ${ }^{159}$ G. R. Snow, ${ }^{159}$ B. Stieger, ${ }^{159}$ J. Dolen, ${ }^{160}$ A. Godshalk, ${ }^{160}$ C. Harrington, ${ }^{160}$ I. Iashvili, ${ }^{160}$ D. Nguyen, ${ }^{160}$ A. Parker, ${ }^{160}$ S. Rappoccio, ${ }^{160}$ B. Roozbahani, ${ }^{160}$ G. Alverson, ${ }^{161}$ E. Barberis, ${ }^{161}$ C. Freer, ${ }^{161}$ A. Hortiangtham, ${ }^{161}$ A. Massironi, ${ }^{161}$ D. M. Morse ${ }^{161}$ T. Orimoto, ${ }^{161}$ R. Teixeira De Lima, ${ }^{161}$ D. Trocino, ${ }^{161}$ T. Wamorkar, ${ }^{161}$ B. Wang, ${ }^{161}$ A. Wisecarver, ${ }^{161}$ D. Wood, ${ }^{161}$ S. Bhattacharya,${ }^{162}$ O. Charaf, ${ }^{162}$ K. A. Hahn, ${ }^{162}$ N. Mucia, ${ }^{162}$ N. Odell, ${ }^{162}$ M. H. Schmitt, ${ }^{162}$ K. Sung, ${ }^{162}$ M. Trovato, ${ }^{162}$ M. Velasco, ${ }^{162}$ R. Bucci, ${ }^{163}$ N. Dev,${ }^{163}$ M. Hildreth, ${ }^{163}$ K. Hurtado Anampa, ${ }^{163}$ C. Jessop, ${ }^{163}$ D. J. Karmgard, ${ }^{163}$ N. Kellams, ${ }^{163}$ K. Lannon, ${ }^{163}$ W. Li, ${ }^{163}$ N. Loukas, ${ }^{163}$ N. Marinelli, ${ }^{163}$ F. Meng, ${ }^{163}$ C. Mueller, ${ }^{163}$ Y. Musienko, ${ }^{163, \mathrm{kk}}$ M. Planer ${ }^{163}$ A. Reinsvold ${ }^{163}$ R. Ruchti, ${ }^{163}$ P. Siddireddy, ${ }^{163}$ G. Smith, ${ }^{163}$ S. Taroni, ${ }^{163}$ M. Wayne, ${ }^{163}$ A. Wightman, ${ }^{163}$ M. Wolf, ${ }^{163}$ A. Woodard, ${ }^{163}$ J. Alimena, ${ }^{164}$ L. Antonelli, ${ }^{164}$ B. Bylsma, ${ }^{164}$ L. S. Durkin, ${ }^{164}$ S. Flowers, ${ }^{164}$ B. Francis, ${ }^{164}$ A. Hart, ${ }^{164}$ C. Hill, ${ }^{164}$ W. Ji ${ }^{164}$ T. Y. Ling, ${ }^{164}$ B. Liu, ${ }^{164}$ W. Luo, ${ }^{164}$ B. L. Winer, ${ }^{164}$ H. W. Wulsin, ${ }^{164}$ S. Cooperstein, ${ }^{165}$ O. Driga, ${ }^{165}$ P. Elmer, ${ }^{165}$ J. Hardenbrook, ${ }^{165}$ P. Hebda, ${ }^{165}$ S. Higginbotham, ${ }^{165}$ A. Kalogeropoulos, ${ }^{165}$ D. Lange, ${ }^{165}$ J. Luo, ${ }^{165}$ D. Marlow, ${ }^{165}$ K. Mei, ${ }^{165}$ I. Ojalvo, ${ }^{165}$ J. Olsen, ${ }^{165}$ C. Palmer, ${ }^{165}$ P. Piroué, ${ }^{165}$ D. Stickland, ${ }^{165}$ C. Tully, ${ }^{165}$ S. Malik, ${ }^{166}$ S. Norberg, ${ }^{166}$ A. Barker,${ }^{167}$ V. E. Barnes, ${ }^{167}$ S. Das, ${ }^{167}$ S. Folgueras, ${ }^{167}$ L. Gutay, ${ }^{167}$ M. Jones, ${ }^{167}$ A. W. Jung, ${ }^{167}$ A. Khatiwada ${ }^{167}$ D. H. Miller, ${ }^{167}$ N. Neumeister, ${ }^{167}$ C. C. Peng, ${ }^{167}$ H. Qiu, ${ }^{167}$ J. F. Schulte, ${ }^{167}$ J. Sun, ${ }^{167}$ F. Wang, ${ }^{167}$ R. Xiao, ${ }^{167}$ W. Xie, ${ }^{167}$ T. Cheng, ${ }^{168}$ N. Parashar, ${ }^{168}$ J. Stupak, ${ }^{168}$ Z. Chen, ${ }^{169}$ K. M. Ecklund, ${ }^{169}$ S. Freed, ${ }^{169}$ F. J. M. Geurts, ${ }^{169}$ M. Guilbaud, ${ }^{169}$ M. Kilpatrick, ${ }^{169}$ W. Li, ${ }^{169}$ B. Michlin, ${ }^{169}$ B. P. Padley, ${ }^{169}$ J. Roberts, ${ }^{169}$ J. Rorie, ${ }^{169}$ W. Shi, ${ }^{169}$ Z. Tu, ${ }^{169}$ J. Zabel, ${ }^{169}$ A. Zhang, ${ }^{169}$ A. Bodek,${ }^{170}$ P. de Barbaro, ${ }^{170}$ R. Demina, ${ }^{170}$

Y. t. Duh, ${ }^{170}$ T. Ferbel, ${ }^{170}$ M. Galanti, ${ }^{170}$ A. Garcia-Bellido, ${ }^{170}$ J. Han,${ }^{170}$ O. Hindrichs, ${ }^{170}$ A. Khukhunaishvili, ${ }^{170}$ K. H. Lo, ${ }^{170}$ P. Tan,${ }^{170}$ M. Verzetti, ${ }^{170}$ R. Ciesielski, ${ }^{171}$ K. Goulianos, ${ }^{171}$ C. Mesropian, ${ }^{171}$ A. Agapitos, ${ }^{172}$ J. P. Chou, ${ }^{172}$ Y. Gershtein, ${ }^{172}$ T. A. Gómez Espinosa, ${ }^{172}$ E. Halkiadakis, ${ }^{172}$ M. Heindl, ${ }^{172}$ E. Hughes, ${ }^{172}$ S. Kaplan, ${ }^{172}$

R. Kunnawalkam Elayavalli, ${ }^{172}$ S. Kyriacou, ${ }^{172}$ A. Lath, ${ }^{172}$ R. Montalvo, ${ }^{172}$ K. Nash ${ }^{172}$ M. Osherson, ${ }^{172}$ H. Saka, ${ }^{172}$ S. Salur, ${ }^{172}$ S. Schnetzer, ${ }^{172}$ D. Sheffield, ${ }^{172}$ S. Somalwar, ${ }^{172}$ R. Stone, ${ }^{172}$ S. Thomas, ${ }^{172}$ P. Thomassen, ${ }^{172}$ M. Walker, ${ }^{172}$

A. G. Delannoy, ${ }^{173}$ J. Heideman, ${ }^{173}$ G. Riley, ${ }^{173}$ K. Rose, ${ }^{173}$ S. Spanier ${ }^{173}$ K. Thapa, ${ }^{173}$ O. Bouhali,,${ }^{174, \text { rrr }}$

A. Castaneda Hernandez, ${ }^{174, \text { rrr }}$ A. Celik, ${ }^{174}$ M. Dalchenko, ${ }^{174}$ M. De Mattia, ${ }^{174}$ A. Delgado, ${ }^{174}$ S. Dildick, ${ }^{174}$ R. Eusebi, ${ }^{174}$ J. Gilmore, ${ }^{174}$ T. Huang, ${ }^{174}$ T. Kamon, ${ }^{174, \text { sss }}$ R. Mueller, ${ }^{174}$ Y. Pakhotin, ${ }^{174}$ R. Patel,${ }^{174}$ A. Perloff,,${ }^{174}$ L. Perniè, ${ }^{174}$

D. Rathjens, ${ }^{174}$ A. Safonov, ${ }^{174}$ A. Tatarinov, ${ }^{174}$ K. A. Ulmer, ${ }^{174}$ N. Akchurin, ${ }^{175}$ J. Damgov, ${ }^{175}$ F. De Guio, ${ }^{175}$

P. R. Dudero, ${ }^{175}$ J. Faulkner, ${ }^{175}$ E. Gurpinar, ${ }^{175}$ S. Kunori, ${ }^{175}$ K. Lamichhane, ${ }^{175}$ S. W. Lee, ${ }^{175}$ T. Libeiro, ${ }^{175}$ T. Mengke, ${ }^{175}$ S. Muthumuni, ${ }^{175}$ T. Peltola, ${ }^{175}$ S. Undleeb, ${ }^{175} \mathrm{I}$. Volobouev, ${ }^{175} \mathrm{Z}$. Wang, ${ }^{175}$ S. Greene, ${ }^{176}$ A. Gurrola, ${ }^{176}$ R. Janjam, ${ }^{176}$ W. Johns, ${ }^{176}$ C. Maguire, ${ }^{176}$ A. Melo, ${ }^{176}$ H. Ni ${ }^{176}$ K. Padeken, ${ }^{176}$ P. Sheldon, ${ }^{176}$ S. Tuo, ${ }^{176}$ J. Velkovska, ${ }^{176}$ Q. Xu, ${ }^{176}$ M. W. Arenton, ${ }^{177}$ P. Barria, ${ }^{177}$ B. Cox,${ }^{177}$ R. Hirosky, ${ }^{177}$ M. Joyce,${ }^{177}$ A. Ledovskoy, ${ }^{177}$ H. Li, ${ }^{177}$ C. Neu, ${ }^{177}$

T. Sinthuprasith, ${ }^{177}$ Y. Wang, ${ }^{177}$ E. Wolfe, ${ }^{177}$ F. Xia, ${ }^{177}$ R. Harr,${ }^{178}$ P. E. Karchin, ${ }^{178}$ N. Poudyal, ${ }^{178}$ J. Sturdy,${ }^{178}$ P. Thapa, ${ }^{178}$ 


\title{
S. Zaleski, ${ }^{178}$ M. Brodski, ${ }^{179}$ J. Buchanan, ${ }^{179}$ C. Caillol, ${ }^{179}$ D. Carlsmith, ${ }^{179}$ S. Dasu, ${ }^{179}$ L. Dodd, ${ }^{179}$ S. Duric, ${ }^{179}$
} B. Gomber, ${ }^{179}$ M. Grothe, ${ }^{179}$ M. Herndon ${ }^{179}$ A. Hervé, ${ }^{179}$ U. Hussain, ${ }^{179}$ P. Klabbers, ${ }^{179}$ A. Lanaro, ${ }^{179}$ A. Levine, ${ }^{179}$ K. Long, ${ }^{179}$ R. Loveless, ${ }^{179}$ T. Ruggles, ${ }^{179}$ A. Savin, ${ }^{179}$ N. Smith, ${ }^{179}$ W. H. Smith, ${ }^{179}$ D. Taylor, ${ }^{179}$ and N. Woods ${ }^{179}$

\section{(CMS Collaboration)}

\author{
${ }^{1}$ Yerevan Physics Institute, Yerevan, Armenia \\ ${ }^{2}$ Institut für Hochenergiephysik, Wien, Austria \\ ${ }^{3}$ Institute for Nuclear Problems, Minsk, Belarus \\ ${ }^{4}$ Universiteit Antwerpen, Antwerpen, Belgium \\ ${ }^{5}$ Vrije Universiteit Brussel, Brussel, Belgium \\ ${ }^{6}$ Université Libre de Bruxelles, Bruxelles, Belgium \\ ${ }^{7}$ Ghent University, Ghent, Belgium \\ ${ }^{8}$ Université Catholique de Louvain, Louvain-la-Neuve, Belgium \\ ${ }^{9}$ Centro Brasileiro de Pesquisas Fisicas, Rio de Janeiro, Brazil \\ ${ }^{10}$ Universidade do Estado do Rio de Janeiro, Rio de Janeiro, Brazil \\ ${ }^{11 a}$ Universidade Estadual Paulista, São Paulo, Brazil \\ ${ }^{11 \mathrm{~b}}$ Universidade Federal do ABC, São Paulo, Brazil \\ ${ }^{12}$ Institute for Nuclear Research and Nuclear Energy, Bulgarian Academy of Sciences, Sofia, Bulgaria \\ ${ }^{13}$ University of Sofia, Sofia, Bulgaria \\ ${ }^{14}$ Beihang University, Beijing, China \\ ${ }^{15}$ Institute of High Energy Physics, Beijing, China \\ ${ }^{16}$ State Key Laboratory of Nuclear Physics and Technology, Peking University, Beijing, China \\ ${ }^{17}$ Tsinghua University, Beijing, China \\ ${ }^{18}$ Universidad de Los Andes, Bogota, Colombia \\ ${ }^{19}$ University of Split, Faculty of Electrical Engineering, Mechanical Engineering and Naval Architecture, \\ Split, Croatia \\ ${ }^{20}$ University of Split, Faculty of Science, Split, Croatia \\ ${ }^{21}$ Institute Rudjer Boskovic, Zagreb, Croatia \\ ${ }^{22}$ University of Cyprus, Nicosia, Cyprus \\ ${ }^{23}$ Charles University, Prague, Czech Republic \\ ${ }^{24}$ Universidad San Francisco de Quito, Quito, Ecuador \\ ${ }^{25}$ Academy of Scientific Research and Technology of the Arab Republic of Egypt, \\ Egyptian Network of High Energy Physics, Cairo, Egypt \\ ${ }^{26}$ National Institute of Chemical Physics and Biophysics, Tallinn, Estonia \\ ${ }^{27}$ Department of Physics, University of Helsinki, Helsinki, Finland \\ ${ }^{28}$ Helsinki Institute of Physics, Helsinki, Finland \\ ${ }^{29}$ Lappeenranta University of Technology, Lappeenranta, Finland \\ ${ }^{30} I R F U, C E A$, Université Paris-Saclay, Gif-sur-Yvette, France \\ ${ }^{31}$ Laboratoire Leprince-Ringuet, Ecole polytechnique, CNRS/IN2P3, Université Paris-Saclay, \\ Palaiseau, France \\ ${ }^{32}$ Université de Strasbourg, CNRS, IPHC UMR 7178, F-67000 Strasbourg, France \\ ${ }^{33}$ Centre de Calcul de l'Institut National de Physique Nucleaire et de Physique des Particules, CNRS/ \\ IN2P3, Villeurbanne, France \\ ${ }^{34}$ Université de Lyon, Université Claude Bernard Lyon 1, CNRS-IN2P3, Institut de Physique Nucléaire de \\ Lyon, Villeurbanne, France \\ ${ }^{35}$ Georgian Technical University, Tbilisi, Georgia \\ ${ }^{36}$ Tbilisi State University, Tbilisi, Georgia \\ ${ }^{37}$ RWTH Aachen University, I. Physikalisches Institut, Aachen, Germany \\ ${ }^{38}$ RWTH Aachen University, III. Physikalisches Institut A, Aachen, Germany \\ ${ }^{39}$ RWTH Aachen University, III. Physikalisches Institut B, Aachen, Germany \\ ${ }^{40}$ Deutsches Elektronen-Synchrotron, Hamburg, Germany \\ ${ }^{41}$ University of Hamburg, Hamburg, Germany \\ ${ }^{42}$ Institut für Experimentelle Kernphysik, Karlsruhe, Germany \\ ${ }^{43}$ Institute of Nuclear and Particle Physics (INPP), NCSR Demokritos, Aghia Paraskevi, Greece \\ ${ }^{44}$ National and Kapodistrian University of Athens, Athens, Greece \\ ${ }^{45}$ National Technical University of Athens, Athens, Greece \\ ${ }^{46}$ University of Ioánnina, Ioánnina, Greece
}


${ }^{47}$ MTA-ELTE Lendület CMS Particle and Nuclear Physics Group,

Eötvös Loránd University, Budapest, Hungary

${ }^{48}$ Wigner Research Centre for Physics, Budapest, Hungary

${ }^{49}$ Institute of Nuclear Research ATOMKI, Debrecen, Hungary

${ }^{50}$ Institute of Physics, University of Debrecen, Debrecen, Hungary

${ }^{51}$ Indian Institute of Science (IISc), Bangalore, India

${ }^{52}$ National Institute of Science Education and Research, Bhubaneswar, India

${ }^{53}$ Panjab University, Chandigarh, India

${ }^{54}$ University of Delhi, Delhi, India

${ }^{55}$ Saha Institute of Nuclear Physics, HBNI, Kolkata, India

${ }^{56}$ Indian Institute of Technology Madras, Madras, India

${ }^{57}$ Bhabha Atomic Research Centre, Mumbai, India

${ }^{58}$ Tata Institute of Fundamental Research-A, Mumbai, India

${ }^{59}$ Tata Institute of Fundamental Research-B, Mumbai, India

${ }^{60}$ Indian Institute of Science Education and Research (IISER), Pune, India

${ }^{61}$ Institute for Research in Fundamental Sciences (IPM), Tehran, Iran

${ }^{62}$ University College Dublin, Dublin, Ireland

${ }^{63 \mathrm{a}}$ INFN Sezione di Bari, Bari, Italy

${ }^{63 \mathrm{~b}}$ Università di Bari, Bari, Italy

${ }^{63 \mathrm{c}}$ Politecnico di Bari, Bari, Italy

${ }^{64 \mathrm{a}}$ INFN Sezione di Bologna, Bologna, Italy

${ }^{64 \mathrm{~b}}$ Università di Bologna, Bologna, Italy

${ }^{65 a}$ INFN Sezione di Catania, Catania, Italy

${ }^{65 \mathrm{~b}}$ Università di Catania, Catania, Italy

${ }^{66 \mathrm{a}}$ INFN Sezione di Firenze, Firenze, Italy

${ }^{66 \mathrm{~b}}$ Università di Firenze, Firenze, Italy

${ }^{67}$ INFN Laboratori Nazionali di Frascati, Frascati, Italy

${ }^{68 a}$ INFN Sezione di Genova, Genova, Italy

${ }^{68 \mathrm{~b}}$ Università di Genova, Genova, Italy

${ }^{69 a}$ INFN Sezione di Milano-Bicocca

${ }^{69 \mathrm{~b}}$ Università di Milano-Bicocca

${ }^{70 a}$ INFN Sezione di Napoli, Napoli, Italy

${ }^{70 \mathrm{~b}}$ Università di Napoli 'Federico II', Napoli, Italy

${ }^{70 c}$ Università della Basilicata, Potenza, Italy

${ }^{70 \mathrm{~d}}$ Università G. Marconi, Roma, Italy

${ }^{71 a}$ INFN Sezione di Padova, Padova, Italy

${ }^{71 \mathrm{~b}}$ Università di Padova, Padova, Italy

${ }^{71 \mathrm{c}}$ Università di Trento, Trento, Italy

${ }^{72 a}$ INFN Sezione di Pavia, Pavia, Italy

${ }^{72 \mathrm{~b}}$ Università di Pavia, Pavia, Italy

${ }^{73 a}$ INFN Sezione di Perugia, Perugia, Italy

${ }^{73 b}$ Università di Perugia, Perugia, Italy

${ }^{74 a}$ INFN Sezione di Pisa, Pisa, Italy

${ }^{74 \mathrm{~b}}$ Università di Pisa, Pisa, Italy

${ }^{74 \mathrm{c}}$ Scuola Normale Superiore di Pisa, Pisa, Italy

${ }^{75 a}$ INFN Sezione di Roma, Rome, Italy

${ }^{75 b}$ Sapienza Università di Roma, Rome, Italy

${ }^{76 a}$ INFN Sezione di Torino, Torino, Italy

${ }^{76 \mathrm{~b}}$ Università di Torino, Torino, Italy

${ }^{76 c}$ Università del Piemonte Orientale, Novara, Italy

${ }^{77}$ INFN Sezione di Trieste, Trieste, Italy

${ }^{77 b}$ Università di Trieste, Trieste, Italy

${ }^{78}$ Kyungpook National University, Daegu, Korea

${ }^{79}$ Chonbuk National University, Jeonju, Korea

${ }^{80}$ Chonnam National University, Institute for Universe and Elementary Particles, Kwangju, Korea

${ }^{81}$ Hanyang University, Seoul, Korea

${ }^{82}$ Korea University, Seoul, Korea

${ }^{83}$ Seoul National University, Seoul, Korea

${ }^{84}$ University of Seoul, Seoul, Korea

${ }^{85}$ Sungkyunkwan University, Suwon, Korea 
${ }^{86}$ Vilnius University, Vilnius, Lithuania

${ }^{87}$ National Centre for Particle Physics, Universiti Malaya, Kuala Lumpur, Malaysia

${ }^{88}$ Centro de Investigacion y de Estudios Avanzados del IPN, Mexico City, Mexico

${ }^{89}$ Universidad Iberoamericana, Mexico City, Mexico

${ }^{90}$ Benemerita Universidad Autonoma de Puebla, Puebla, Mexico

${ }^{91}$ Universidad Autónoma de San Luis Potosí, San Luis Potosí, Mexico

${ }^{92}$ University of Auckland, Auckland, New Zealand

${ }^{93}$ University of Canterbury, Christchurch, New Zealand

${ }^{94}$ National Centre for Physics, Quaid-I-Azam University, Islamabad, Pakistan

${ }^{95}$ National Centre for Nuclear Research, Swierk, Poland

${ }^{96}$ Institute of Experimental Physics, Faculty of Physics, University of Warsaw, Warsaw, Poland

${ }^{97}$ Laboratório de Instrumentação e Física Experimental de Partículas, Lisboa, Portugal

${ }^{98}$ Joint Institute for Nuclear Research, Dubna, Russia

${ }^{99}$ Petersburg Nuclear Physics Institute, Gatchina (St. Petersburg), Russia

${ }^{100}$ Institute for Nuclear Research, Moscow, Russia

${ }^{101}$ Institute for Theoretical and Experimental Physics, Moscow, Russia

${ }^{102}$ Moscow Institute of Physics and Technology, Moscow, Russia

${ }^{103}$ National Research Nuclear University 'Moscow Engineering Physics Institute' (MEPhI), Moscow, Russia

${ }^{104}$ P.N. Lebedev Physical Institute, Moscow, Russia

${ }^{105}$ Skobeltsyn Institute of Nuclear Physics, Lomonosov Moscow State University, Moscow, Russia

${ }^{106}$ Novosibirsk State University (NSU), Novosibirsk, Russia

${ }^{107}$ State Research Center of Russian Federation, Institute for High Energy Physics of NRC

"Kurchatov Institute”, Protvino, Russia

${ }^{108}$ University of Belgrade, Faculty of Physics and Vinca Institute of Nuclear Sciences, Belgrade, Serbia

${ }^{109}$ Centro de Investigaciones Energéticas Medioambientales y Tecnológicas (CIEMAT), Madrid, Spain

${ }^{110}$ Universidad Autónoma de Madrid, Madrid, Spain

${ }^{111}$ Universidad de Oviedo, Oviedo, Spain

${ }^{112}$ Instituto de Física de Cantabria (IFCA), CSIC-Universidad de Cantabria, Santander, Spain

${ }^{113}$ CERN, European Organization for Nuclear Research, Geneva, Switzerland

${ }^{114}$ Paul Scherrer Institut, Villigen, Switzerland

${ }^{115}$ ETH Zurich-Institute for Particle Physics and Astrophysics (IPA), Zurich, Switzerland

${ }^{116}$ Universität Zürich, Zurich, Switzerland

${ }^{117}$ National Central University, Chung-Li, Taiwan

${ }^{118}$ National Taiwan University (NTU), Taipei, Taiwan

${ }^{119}$ Chulalongkorn University, Faculty of Science, Department of Physics, Bangkok, Thailand

${ }^{120}$ Cukurova University, Physics Department, Science and Art Faculty, Adana, Turkey

${ }^{121}$ Middle East Technical University, Physics Department, Ankara, Turkey

${ }^{122}$ Bogazici University, Istanbul, Turkey

${ }^{123}$ Istanbul Technical University, Istanbul, Turkey

${ }^{124}$ Institute for Scintillation Materials of National Academy of Science of Ukraine, Kharkov, Ukraine

${ }^{125}$ National Scientific Center, Kharkov Institute of Physics and Technology, Kharkov, Ukraine

${ }^{126}$ University of Bristol, Bristol, United Kingdom

${ }^{127}$ Rutherford Appleton Laboratory, Didcot, United Kingdom

${ }^{128}$ Imperial College, London, United Kingdom

${ }^{129}$ Brunel University, Uxbridge, United Kingdom

${ }^{130}$ Baylor University, Waco, USA

${ }^{131}$ Catholic University of America, Washington DC, USA

${ }^{132}$ The University of Alabama, Tuscaloosa, USA

${ }^{133}$ Boston University, Boston, USA

${ }^{134}$ Brown University, Providence, USA

${ }^{135}$ University of California, Davis, Davis, USA

${ }^{136}$ University of California, Los Angeles, USA

${ }^{137}$ University of California, Riverside, Riverside, USA

${ }^{138}$ University of California, San Diego, La Jolla, USA

${ }^{139}$ University of California, Santa Barbara-Department of Physics, Santa Barbara, USA

${ }^{140}$ California Institute of Technology, Pasadena, USA

${ }^{141}$ Carnegie Mellon University, Pittsburgh, USA

${ }^{142}$ University of Colorado Boulder, Boulder, USA

${ }^{143}$ Cornell University, Ithaca, USA 


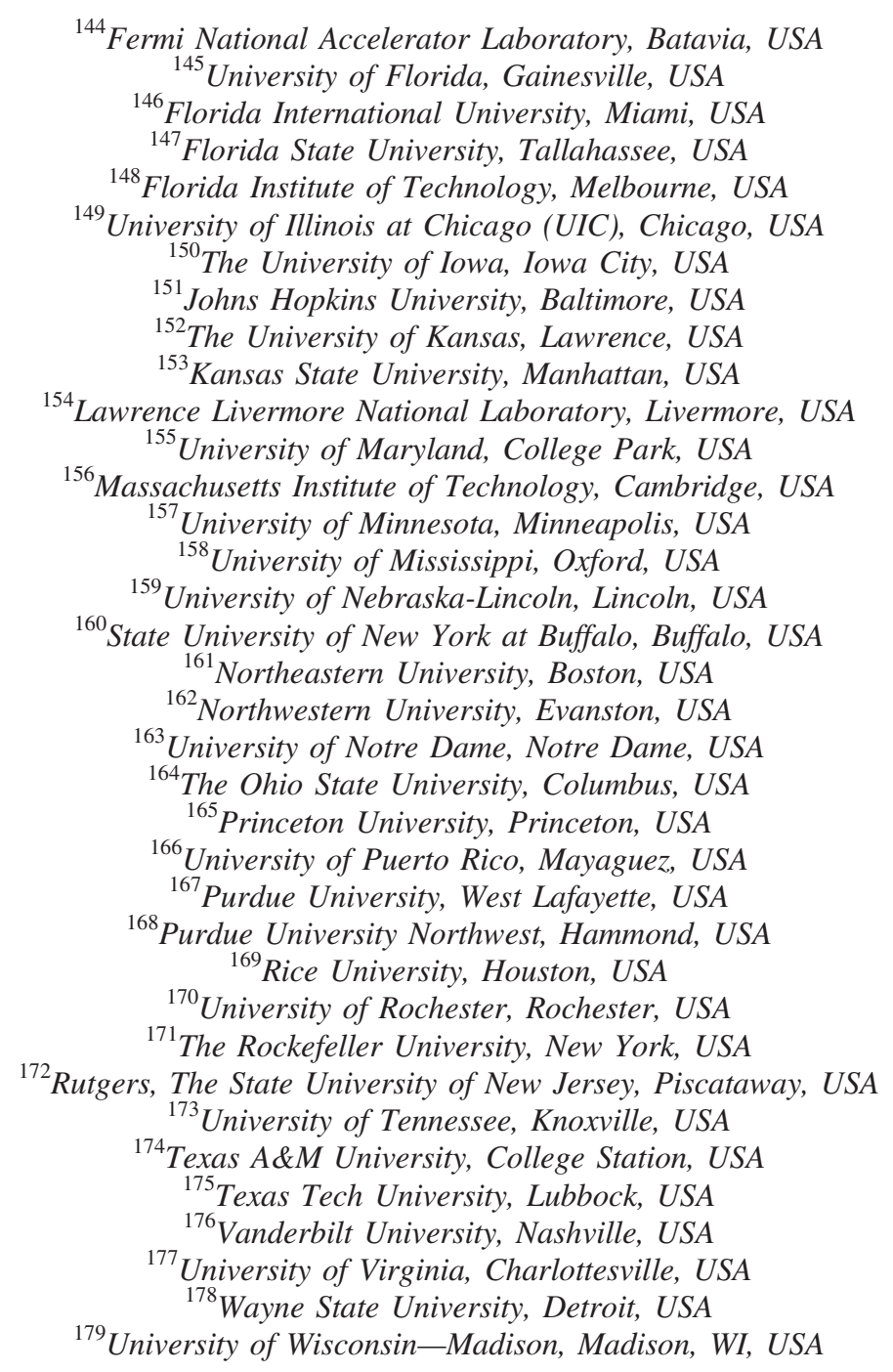

${ }^{\mathrm{a}}$ Deceased.

${ }^{\mathrm{b}}$ Also at Vienna University of Technology, Vienna, Austria.

${ }^{c}$ Also at IRFU, CEA, Université Paris-Saclay, Gif-sur-Yvette, France.

${ }^{\mathrm{d}}$ Also at Universidade Estadual de Campinas, Campinas, Brazil.

${ }^{\mathrm{e}}$ Also at Federal University of Rio Grande do Sul, Porto Alegre, Brazil.

${ }^{\mathrm{f}}$ Also at Université Libre de Bruxelles, Bruxelles, Belgium.

${ }^{\mathrm{g}}$ Also at Institute for Theoretical and Experimental Physics, Moscow, Russia.

${ }^{\mathrm{h}}$ Also at Joint Institute for Nuclear Research, Dubna, Russia.

${ }^{\mathrm{i}}$ Also at Suez University, Suez, Egypt.

${ }^{\mathrm{j}}$ Also at British University in Egypt, Cairo, Egypt.

${ }^{\mathrm{k}}$ Also at Zewail City of Science and Technology, Zewail, Egypt.

${ }^{1}$ Also at Department of Physics, King Abdulaziz University, Jeddah, Saudi Arabia.

${ }^{\mathrm{m}}$ Also at Université de Haute Alsace, Mulhouse, France.

${ }^{\mathrm{n}}$ Also at Skobeltsyn Institute of Nuclear Physics, Lomonosov Moscow State University, Moscow, Russia.

${ }^{\circ}$ Also at Tbilisi State University, Tbilisi, Georgia.

${ }^{\mathrm{p}}$ Also at CERN, European Organization for Nuclear Research, Geneva, Switzerland.

${ }^{\mathrm{q}}$ Also at RWTH Aachen University, III. Physikalisches Institut A, Aachen, Germany.

${ }^{\mathrm{r}}$ Also at University of Hamburg, Hamburg, Germany.

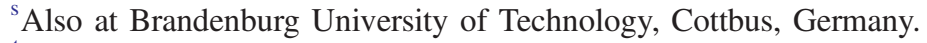

${ }^{t}$ Also at MTA-ELTE Lendület CMS Particle and Nuclear Physics Group, Eötvös Loránd University, Budapest, Hungary.

uAlso at Institute of Nuclear Research ATOMKI, Debrecen, Hungary.

${ }^{v}$ Also at Institute of Physics, University of Debrecen, Debrecen, Hungary.

${ }^{\mathrm{w}}$ Also at IIT Bhubaneswar, Bhubaneswar, India. 
${ }^{\mathrm{x}}$ Also at Institute of Physics, Bhubaneswar, India.

${ }^{y}$ Also at University of Visva-Bharati, Santiniketan, India.

${ }^{\mathrm{z}}$ Also at University of Ruhuna, Matara, Sri Lanka.

${ }^{\text {aa }}$ Also at Isfahan University of Technology, Isfahan, Iran.

${ }^{\mathrm{bb}}$ Also at Yazd University, Yazd, Iran.

${ }^{\mathrm{cc}}$ Also at Plasma Physics Research Center, Science and Research Branch, Islamic Azad University, Tehran, Iran.

${ }^{\mathrm{dd}}$ Also at Università degli Studi di Siena, Siena, Italy.

${ }^{e e}$ Also at INFN Sezione di Milano-Bicocca, Università di Milano-Bicocca, Milano, Italy.

${ }^{\mathrm{ff}}$ Also at Purdue University, West Lafayette, USA.

${ }^{\mathrm{gg}}$ Also at International Islamic University of Malaysia, Kuala Lumpur, Malaysia.

${ }^{\text {hh }}$ Also at Malaysian Nuclear Agency, MOSTI, Kajang, Malaysia.

${ }^{i i}$ Also at Consejo Nacional de Ciencia y Tecnología, Mexico city, Mexico.

${ }^{\mathrm{jj}}$ Also at Warsaw University of Technology, Institute of Electronic Systems, Warsaw, Poland.

${ }^{\mathrm{kk}}$ Also at Institute for Nuclear Research, Moscow, Russia.

${ }^{11}$ Also at National Research Nuclear University 'Moscow Engineering Physics Institute' (MEPhI), Moscow, Russia.

${ }^{\mathrm{mm}}$ Also at St. Petersburg State Polytechnical University, St. Petersburg, Russia.

${ }^{n n}$ Also at University of Florida, Gainesville, USA.

${ }^{\circ o}$ Also at P.N. Lebedev Physical Institute, Moscow, Russia.

${ }^{\mathrm{pp}}$ Also at California Institute of Technology, Pasadena, USA.

${ }^{\mathrm{qq}}$ Also at Budker Institute of Nuclear Physics, Novosibirsk, Russia.

${ }^{\mathrm{rr}}$ Also at Faculty of Physics, University of Belgrade, Belgrade, Serbia.

${ }^{\text {ss }}$ Also at University of Belgrade, Faculty of Physics and Vinca Institute of Nuclear Sciences, Belgrade, Serbia.

${ }^{\text {tt}}$ Also at Scuola Normale e Sezione dell'INFN, Pisa, Italy.

${ }^{u u}$ Also at National and Kapodistrian University of Athens, Athens, Greece.

${ }^{\mathrm{vv}}$ Also at Riga Technical University, Riga, Latvia.

${ }^{\text {ww }}$ Also at Universität Zürich, Zurich, Switzerland.

${ }^{\mathrm{xx}}$ Also at Stefan Meyer Institute for Subatomic Physics.

${ }^{\mathrm{yy}}$ Also at Adiyaman University, Adiyaman, Turkey.

${ }^{\mathrm{zz}}$ Also at Istanbul Aydin University, Istanbul, Turkey.

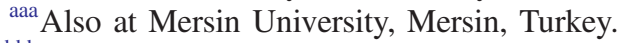

${ }^{\text {bbb }}$ Also at Cag University, Mersin, Turkey.

${ }^{c c c}$ Also at Piri Reis University, Istanbul, Turkey.

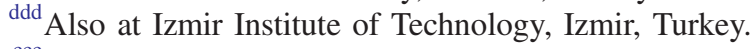

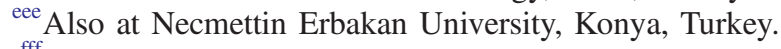

${ }^{\mathrm{fff}}$ Also at Marmara University, Istanbul, Turkey.

${ }^{\text {ggg }}$ Also at Kafkas University, Kars, Turkey.

${ }^{\text {hhh }}$ Also at Istanbul Bilgi University, Istanbul, Turkey.

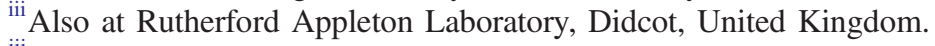

jij Also at School of Physics and Astronomy, University of Southampton, Southampton, United Kingdom.

${ }^{k k k}$ Also at Instituto de Astrofísica de Canarias, La Laguna, Spain.

${ }^{111}$ Also at Utah Valley University, Orem, USA.

${ }^{\mathrm{mmm}}$ Also at Beykent University.

${ }^{n n n}$ Also at Bingol University, Bingol, Turkey.

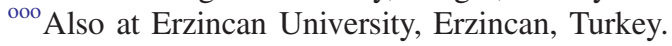

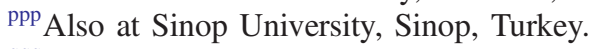

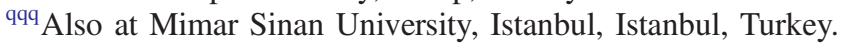

${ }^{\mathrm{rrr}}$ Also at Texas A\&M University at Qatar, Doha, Qatar.

${ }^{\text {sss }}$ Also at Kyungpook National University, Daegu, Korea. 